\title{
Ramified Higher-Order Unification
}

\author{
Jean Goubault-Larrecq \\ Institut für Logik, Komplexität und Deduktionssysteme \\ Universität Karlsruhe, D-76128 Karlsruhe*† \\ Jean.Goubault@frcl.bull.fr
}

August 29, 1996

\begin{abstract}
While unification in the simple theory of types (a.k.a. higher-order logic) is undecidable $\Gamma$ we show that unification in the pure ramified theory of types with integer levels is decidable. But the pure ramified theory of types cannot express even the simplest formulas of logic. The impure ramified type theory has an undecidable unification problem even at order 2. However $\Gamma$ the decidability result for the pure subsystem indicates that unification should fail to terminate less often than general higher-order unification. We present applications to two expressive subsystems of secondorder Peano arithmetic $\Gamma A C A_{0}$ and $\Pi_{k}^{1}-\mathrm{CA}_{0}$.
\end{abstract}

\section{Introduction}

Higher-order logic is one of the most expressive formalisms in which we can express and prove theorems. Bertrand Russell proposed two ways of formalizing it. In ramified type theory [WR27] Texpressions are stratified in a double hierarchy of types (individuals $\Gamma$ sets $\Gamma$ sets of sets $\Gamma$ etc.) and of predication levels (corresponding to times of definition $\Gamma$ and called orders by them); but the resulting logical system is too weak to found mathematics Tand so-called reducibility axioms are called for. In simple type theory levels are dispensed with; the resulting language is Church's simply-typed $\lambda$-calculus with additional constants representing logical and non-logical symbols [Chu40]. (See also [And86].)

A central problem in automated theorem proving and logic programming is that of unificationTi.e. deciding whether two terms have a common instance. Unfortunately $\mathrm{\Gamma}$ unification in the simple theory of

\footnotetext{
*Research funded by the HCM grant 7532.7-06 from the European Union.

†On leave from Bull Corporate Research Center, rue Jean Jaurès, F-78340 Les Clayes sous Bois.
}

types is undecidable even at order 2 [Gol81]. Our original idea was to refine the study of higher-order unification by reintroducing ramification. This seemed an interesting idea for two complementary reasons: first $\Gamma$ we shall see that ramified higher-order unification in its pure form is decidable; second $\Gamma$ we can $\Gamma$ in theoryГget back the full power of simple type theory by introducing Whitehead and Russell's axioms of reducibility. Controlling the use of these axioms would then provide a natural way of controlling the search for unifiers in simple type theory. It also seemed interesting even if we didn't allow for these axioms. Indeed $\Gamma$ ramified type theory has natural restrictions that allow us to formalize weak subsystems of arithmetic like $\mathrm{ACA}_{0}$ or $\mathrm{ATR}_{0} \Gamma$ which are still strong enough to formalize most of mathematics [Sim85]. However T this is only valid in some impure form of ramified type theory for which unification is undecidable again (see Section 7.2). But the decidability result for the pure subsystem indicates that unification should fail to terminate less often than general higher-order unification (in the simple theory of types) $\Gamma$ and so impure ramified type theory should be of practical value in implementing automated proof methods for the subsystems of arithmetic mentioned above.

We may sum up the contributions of this paper as follows. First $\Gamma$ we formalize ramified type theory in both a simple and general wayTwhich does not seem to have been done before. Then Twe prove that unification in this pure system is decidable (Corollary 30 ). We also provide two negative results: first $\Gamma$ that this system is much too weak to formalize any useful fragment of logic; and second 5 that the most natural extension that cures this problem $\Gamma$ ramified type theory with operators $\Gamma$ is undecidable even at order $2-$ Goldfarb's encoding still works (Theorem 31). Finally we argue that the latter theory should be of practical value for automating proof search in $\mathrm{ACA}_{0}$ and related systems. 
The reader willing to learn more about ramification is directed to [LN95] for a modern analysis of what Whitehead and Russell may have meant precisely by ramification and predicativity. Modern accounts of these topics are somewhat differentTand we shall base our study on Hazen's point of view [Haz83]; see also [Cop71TChu76].

The plan of the paper is as follows. We formalize a variant of ramified type theory as a particular type system for the $\lambda$-calculus with equality defined by the $\beta$ and $\eta$ rules. This will take Section 2. We then recall Gallier and Snyder's formalization of Huet's higherorder unification procedure $\Gamma$ and give the rough ideas for adapting it to ramified type theory in Section 3 . The grunt work starts in Section 4 Twhere we establish all needed basic theorems for our ramified calculus. We show that inferring levels of expressions is decidable in Section 5; we need this to check that a unifier in the simple theory of types is in fact one in the ramified theory. Then $\Gamma$ in Section $6 \Gamma$ we replay the ideas expounded in Section 3 in a more formal way. We also deal with the $\beta$-case (without $\eta$ ) in Section 6.2. Finally Twe try to put these ideas to work in Section $7 \Gamma$ and come up with our negative results: the logic is too weak $\Gamma$ and adding operators (see Section 7.2) to cure the problem makes the unification problem undecidable again. Section 8 shows how $\mathrm{ACA}_{0}$ and $\Pi_{k}^{1}-\mathrm{CA}_{0}$ are naturally encoded in the latter impure ramified system. We conclude in Section 9.

\section{Ramified Type Theory}

In this sectionTwe propose a formalization of ramified type theory in a notation that we hope will be familiar to computer scientistsTnamely a type system for Church's $\lambda$-calculus. We also discuss how well it fits with usual views on ramificationTbut bear in mind that our main objective is to find decidable restrictions of higher-order unification through ramification.

Let $S T$ be the algebra of simple types: we have a non-empty set $B$ of base types (among which typically the types $\iota$ of individuals and $o$ of propositions) $\Gamma$ and simple types $\epsilon$ are either base typesTor function types $\epsilon_{1} \rightarrow \epsilon_{2}$.

We define the algebra $R T$ of ramified types by adding levels $\ell$ taken from an initial segment $L$ of the ordinalsTi.e. $L$ is an ordinal. In the sequel $\Gamma$ we shall assume that $L$ is a limit ordinal $\Gamma$ i.e. a non-zero ordinal that is not a successor $\Gamma$ so that for every level $\ell$ there is a greater one in $L$. We shall really be interested in integer levels $\Gamma$ i.e. $L=\omega \Gamma$ as in the original works by Whitehead and Russell. It should be noted that there is nothing wrong in taking for $L$ some seg- ment of the computable ordinals. The idea originates with Gödel and WangTand culminated in Feferman's works on autonomous progressions of predicative systemsTwhere $L=\Gamma_{0}[\mathrm{Haz} 83]$.

Definition 1 (Ramified Types) We define both the set RT of ramified types $\tau$ and their levels $l(\tau)$ by simultaneous induction as follows.

The ramified types are either base types $b$ - and we let $l(b)=0$ - or function types $\tau_{1} \stackrel{\ell}{\rightarrow} \tau_{2}$, where $\tau_{1}$ and $\tau_{2}$ are ramified types and $\ell$ is a level such that $\ell \geq l\left(\tau_{1}\right)-$ then we let $l\left(\tau_{1} \stackrel{\ell}{\rightarrow} \tau_{2}\right)=\max \left(\ell+1, l\left(\tau_{2}\right)\right)$.

We assume that $\rightarrow$ and $\stackrel{\ell}{\rightarrow}$ associate to the right $\Gamma$ so that $\epsilon_{1} \rightarrow \epsilon_{2} \rightarrow \epsilon_{3}$ denotes $\epsilon_{1} \rightarrow\left(\epsilon_{2} \rightarrow \epsilon_{3}\right)$ Гfor example.

The idea is that Twhile the simple type $\epsilon_{1} \rightarrow \epsilon_{2}$ is the type of all total functions from $\epsilon_{1}$ to $\epsilon_{2} \Gamma \tau_{1} \stackrel{\ell}{\rightarrow} \tau_{2}$ is the type of all those functions that act on concepts defined at or after time $\ell$. (Not just at time $\ell$ : contrarily to Whitehead and Russell but following Hazen $\Gamma$ we consider the hierarchy of levels to be cumulative Ti.e. everything at level $\ell$ is also at all higher levels.)

There is a simple connection between simple and ramified types: let the erasing map be defined on ramified types as follows: $E(b)=b$ for all base types $b$ Tand $E\left(\tau_{1} \stackrel{\ell}{\rightarrow} \tau_{2}\right)=E\left(\tau_{1}\right) \rightarrow E\left(\tau_{2}\right)$.

The order $r(\tau)$ of a ramified type $\tau$ is defined inductively as follows. For any base type $b \Gamma r(b)=0$; and $r\left(\tau_{1} \stackrel{\ell}{\rightarrow} \tau_{2}\right)=\max \left(r\left(\tau_{1}\right)+1, r\left(\tau_{2}\right)\right)$. Levels do not play any role here $\Gamma$ and orders of simple types are defined similarly.

We now consider a variant of the $\lambda$-calculus [Bar84] as the basic language for building terms and formulas. The $\lambda$-terms $s \Gamma t \Gamma u \Gamma v \Gamma \ldots$ are variables $x \Gamma y \Gamma$

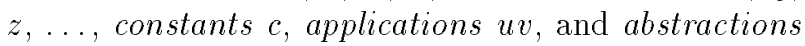
$\lambda x \cdot u$. We assume that application associates to the left $\Gamma$ i.e. uvw denotes ( $u v) w \Gamma$ and that abstractions extend as much right as possible. We also denote by $\mathrm{FVC}(s)$ the set of free variables or constants of $s$. (Think of constants as ordinary variables of the $\lambda$-calculus with the difference that we cannot substitute any term for them.) To avoid variable capture problems Twe also adopt Barendregt's convention that no free variables occurs bound $\Gamma$ and that no two occurrences of $\lambda$ bind the same variables (this involves some renaming of bound variables). Substitutions $\sigma$ are finite maps from variables $x_{1} \Gamma \ldots \Gamma x_{n}$ to $\lambda$-terms $v_{1} \Gamma \ldots \Gamma v_{n} \Gamma$ are are written $\left[v_{1} / x_{1}, \ldots, v_{n} / x_{n}\right]$. The application $u \sigma$ of the substitution $\sigma$ to the term $u$ is then defined straightforwardly as textual substitution; the composition $\sigma \sigma^{\prime}$ is the only substitution such 
that $u\left(\sigma \sigma^{\prime}\right)=(u \sigma) \sigma^{\prime}$ for all terms $u$; and the domain dom $\sigma$ of $\sigma$ is the set of variables $x$ such that $x \neq x \sigma$.

We consider that the variables and constants have a uniquely determined ramified type $\tau$ and level $\ell$ such that $\ell \geq l(\tau)$. To emphasize it $\Gamma$ we shall write $x_{\tau}^{\ell}$ instead of $x$ to state that $x$ is given type $\tau$ and level $\ell$ (resp. $c_{\tau}^{\ell}$ instead of $c$ ). When context permits $\Gamma$ we shall leave these annotations implicit.

We consider that two terms that differ only by a change of bound variables of the same simple type are equal ( $\alpha$-renaming) Гi.e. $\lambda x_{\tau}^{\ell} \cdot u=\lambda y_{\tau^{\prime}}^{\ell^{\prime}} \cdot u\left[y_{\tau^{\prime}}^{\ell^{\prime}} / x_{\tau}^{\ell}\right]$ provided that $E(\tau)=E\left(\tau^{\prime}\right)$. We shall explain this shortly.

The calculus is then endowed with the following two standard reduction relations:

(B) $(\lambda x \cdot u) v \rightarrow u[v / x]$

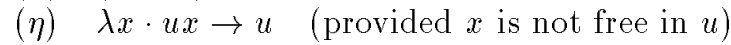

We write $\Leftrightarrow \biguplus_{\beta}$ the smallest relation on terms containing $(\beta)$ and stable by context application. More formally[call a context any term with exactly one hole []: the contexts $\mathcal{C}$ are described by the grammar:

$$
\mathcal{C}::=[]|\mathcal{C} T| T \mathcal{C} \mid \lambda X \cdot \mathcal{C}
$$

where $T$ is the set of terms and $X$ the set of variables. We note $\mathcal{C}[t]$ the result of replacing the hole in $\mathcal{C}$ with the term (or context) $t$. The $\Leftrightarrow \rightarrow_{\beta}$ relation is then defined as $\mathcal{C}[s] \Leftrightarrow \rightarrow_{\beta} \mathcal{C}[t]$ for every context $\mathcal{C} \Gamma$ and every $\beta$-redex $s(=(\lambda x \cdot u) v)$ whose contractum $(=u[v / x])$ is $t$. We write $\stackrel{*}{\leftrightarrow} \beta$ its reflexive transitive closure $\Gamma \stackrel{+}{\Leftrightarrow} \beta$ its transitive closure $\Gamma$ and $=_{\beta}$ its reflexive symmetric transitive closure. Similarly with $\Leftrightarrow \beta_{\eta} \Gamma \stackrel{*}{\leftrightarrow} \beta \eta \Gamma \stackrel{+}{\leftrightarrow} \beta \eta$ and $=\beta \eta$ Tetc.

It would seem more natural to use a more restricted $\alpha$-renaming rule $\Gamma$ whereby $\lambda x_{\tau}^{\ell} \cdot u=\lambda y_{\tau^{\prime}}^{\ell^{\prime}} \cdot u\left[y_{\tau^{\prime}}^{\ell^{\prime}} / x_{\tau}^{\ell}\right]$ provided that $\tau=\tau^{\prime}$ and $\ell=\ell^{\prime}$. However $\Gamma$ in the presence of $\eta \Gamma$ this is not enough to ensure that the resulting calculus is confluent: if $x$ is not free in $u$ Г then $\lambda x_{\tau}^{\ell} \cdot\left(\lambda y_{\tau}^{\ell^{\prime}} \cdot u\right) x$ reduces by $\beta$ to $\lambda x_{\tau}^{\ell} \cdot u[x / y]$ Tor by $\eta$ to $\lambda y_{\tau}^{\ell^{\prime}} \cdot u$. If the first term is typable $T$ then intuitively $\tau$ and $\tau^{\prime}$ will have the same erasures $\Gamma$ and this is why we consider the less restricted $\alpha$-equivalence to be able to consider them equal.

We shall therefore write abstractions as $\lambda x_{\epsilon} \cdot u \mathrm{~T}$ where $\epsilon$ is the erasure of any ramified type that decorates the bound variable $x \Gamma$ and we forget about the level labelling $x$. Although this seems to defeat the purpose of ramificationTthis is not so. We shall see $\Gamma$ for example $\Gamma$ that higher-order ramified unification with integer levels is decidable Talthough it is not so in the simply-typed case.

For now Tand before we introduce the precise typing rules that we shall userlet us analyze the paradox in intuitive terms. The $\lambda$-notation is short for expressing the comprehension axioms of ramified type theory $\Gamma$ i.e. (omitting a few details) for every term $u$ there is a term $v$ such that $\forall x_{\tau}^{\ell} \cdot(v x \equiv u)$ Twhere $\equiv$ is a suitable notion of equality. If we take $\equiv$ to mean coextensionality (as in [Chu76] Tfor example) Tthen $v_{1} \equiv v_{2}$ if and only if $\forall x_{1}, \ldots, x_{n} \cdot v_{1} x_{1} \ldots x_{n} \Leftrightarrow v_{2} x_{1} \ldots x_{n}$ T where $x_{1} \Gamma \ldots \Gamma x_{n}$ are variable containing all those of $v_{1}$ and $v_{2}$ Tand the usual type and level provisos are obeyed. We have decided to write $\lambda x_{\tau}^{\ell} \cdot u$ for such a term $v$. Now $\Gamma$ assuming that $\equiv$ is a congruence (in particularT passes to context) $\Gamma$ it follows that $\eta$ is valid $\Gamma i . e$. transforms a term into an 三-equal term. The real problem is that $\equiv$ may not be a congruence: indeed $\Gamma$ the rule that $u \equiv v$ implies $f u \equiv f v$ is not deductible in general.

On the other hand $\Gamma$ if we choose for $\equiv$ a form of Leibniz equality namely $v_{1} \equiv v_{2}$ if and only if for every property $P$ of suitable type and level $\Gamma P v_{1}$ holds if and only if $P v_{2}$ holdsTthen there is no reason why $\eta$ equality should hold. But there is no reason why the rule $\xi: u \equiv v$ implies $\lambda x \cdot u \equiv \lambda x \cdot v$ Tshould hold either. I.e. Tif we take the purely intensional route to ramified logics (as described and discussed in [Cop71]) $\Gamma$ the $\lambda$ notation itself is at stake. This is discussed further in [Haz83] TSection 2.

All this boils down to the fact that we have just made a choice of a particular ramified logic $\Gamma$ with rather strong extensionality conditions expressed by the $\eta$ rule and a liberalized $\alpha$-conversion rule. This is not a departure from usual predicative logics Twhile it certainly simplifies the presentation of the framework. We shall examine briefly what happens if we drop the $\eta$ rule in Section 6.2.

Finally Wwe consider the typing rules shown in Figure 1. A decorated type $\tau / \ell$ is just a pair of a typeto-be $\tau$ and a level $\ell$. These rules derive a decorated type $\tau / \ell$ for each expression $u$ as a judgement $u: \tau / \ell$. We shall see (Lemma 2) that Twhenever we can derive $u: \tau / \ell \Gamma$ then $\tau$ will really be a ramified type and $\ell \geq l(\tau)$ will hold. The latter is a condition of ramification $\Gamma$ saying that $u$ cannot be defined earlier than its constituents $\Gamma$ and is analogous to the constraint that we have imposed on function types in Definition 1. FurthermoreTrule ( $A b s$ ) includes explictly the $\alpha$-conversion rule. We might have left it implicit by stating $(A b s)$ as:

$$
\frac{u: \tau_{2} / \ell_{2}}{\lambda x_{\tau_{1}}^{\ell_{1}} \cdot u: \tau_{1} \stackrel{\ell_{1}}{\rightarrow} \tau_{2} / \max \left(\ell_{1}+1, \ell_{2}\right)}
$$

and stating that if $u: \tau / \ell$ is derivable $\Gamma$ then any $\alpha$ variant of $u$ also has type $\tau$ and level $\ell \Gamma$ but we feel that rule $(A b s)$ is more readable. 


$$
\begin{aligned}
& \overline{x_{\tau}^{\ell}: \tau / \ell}(\operatorname{Var}) \\
& \text { ( } x \text { variable or constant) } \\
& \frac{u: \tau_{1} \stackrel{\ell_{1}}{\rightarrow} \tau_{2} / \ell_{2} \quad v: \tau_{1} / \ell_{1}}{u v: \tau_{2} / \ell_{2}}(A p p) \frac{u\left[y_{\tau_{1}}^{\ell_{1}} / x\right]: \tau_{2} / \ell_{2} \quad \epsilon=E\left(\tau_{1}\right) \quad y \notin \mathrm{FVC}(u)}{\lambda x_{\epsilon} \cdot u: \tau_{1} \stackrel{\ell_{1}}{\rightarrow} \tau_{2} / \max \left(\ell_{1}+1, \ell_{2}\right)}(A b s) \\
& \frac{b \text { base type }}{b \sqsubseteq b}(R f l) \\
& \frac{u: \tau / \ell \quad \tau \sqsubseteq \tau^{\prime} \quad \ell \leq \ell^{\prime}}{u: \tau^{\prime} / \ell^{\prime}}(\mathrm{Cml}) \\
& \frac{\tau_{1}^{\prime} \sqsubseteq \tau_{1} \quad \tau_{2} \sqsubseteq \tau_{2}^{\prime} \quad l\left(\tau_{1}^{\prime}\right) \leq \ell^{\prime} \leq \ell}{\tau_{1} \stackrel{\ell}{\rightarrow} \tau_{2} \sqsubseteq \tau_{1}^{\prime} \stackrel{\ell^{\prime}}{\rightarrow} \tau_{2}^{\prime}}(S u b)
\end{aligned}
$$

Figure 1: Typing rules

Type and level annotations on variables and constants are used in rule (Var). Recall that Tfor every variable $x_{\tau}^{\ell} \Gamma \tau$ is assumed to be a ramified type and $\ell$ is assumed to be at least $l(\tau)$. Observe that this implicitly means that $\tau_{1}$ is a ramified type and $\ell_{1} \geq l\left(\tau_{1}\right)$ in rule $(A b s)$. In all other cases $\Gamma$ we do not require the objects $\tau \Gamma \tau_{1} \Gamma \tau_{2} \Gamma \tau_{1}^{\prime} \Gamma \tau_{2}^{\prime}$ to be types $\Gamma$ unless explicitly required (in rule $(R f l)$ ). We shall however see (Lemma 2) that $\Gamma$ in any derivation $\Gamma$ they will be ramified types.

Although it is not assumed in usual texts on ramified type theory [Haz83] $\Gamma$ we also assume a subtype relation $\sqsubseteq$ to express the cumulativity of levels. (We need this in establishing the basic theorems of Section 4.) That the levels are cumulative means that if an object is typed at some level $\ell \Gamma$ then it is also typed at all higher levels. Now TintuitivelyTevery object of type $\tau_{1} \stackrel{\ell}{\rightarrow} \tau_{2}$ is a function that can be applied to objects of level $\ell$ Tor by cumulativity to objects of levels at most $\ell$. So it is consistent to require every object of type $\tau_{1} \stackrel{\ell}{\rightarrow} \tau_{2}$ to be of type $\tau_{1} \stackrel{\ell^{\prime}}{\rightarrow} \tau_{2}$ also $\Gamma$ with $\ell^{\prime} \leq \ell$ : this is the gist of rules $(\mathrm{Cml})$ and $(S u b)$.

Our system is slightly richer than usual ramified systems $\Gamma$ in that everything at some level is also at all higher levels. Usually [Haz83] Tit is just assumed that we may promote $v$ to a higher level when using it as an argument of some function symbol $f$ or of some other term $u$. In particularTfirst-order variables (variables of base types) are usually taken to be at level 0 Twhere 0 is the least level; we allow them to be at any level we wish. This is naturally not essential.

We finally define:

Definition 2 For every ordinals $\alpha \leq \omega$ and $\beta$, we let $\lambda_{\alpha}^{\beta}$ be the set of $\lambda$-terms that are typable in the ramified type theory, and such that:

- all types of subterms have order $<\alpha$;

- the set $L$ of levels is the set of all ordinals $<\beta$.
Therefore The theory of Whitehead and Russell with cumulative levels would be $\lambda_{w}^{\omega}$ Twhile Feferman's systems could be expressed in $\lambda_{2}^{\Gamma_{0}}$.

\section{Unification}

Say that a substitution $\sigma$ is well-typed if and only if it binds every variable $x_{\tau}^{\ell}$ to a term $x \sigma$ of type $\tau / \ell$; equivalently Tto a term $x \sigma$ of type $\tau^{\prime} / \ell^{\prime}$ such that $\tau^{\prime} \sqsubseteq$ $\tau$ and $\ell^{\prime} \leq \ell$. Similarly well-simply-typed if and only if it binds every variable $x_{\tau}^{\ell}$ to a term $x \sigma$ of simple type $E(\tau)$.

We write $\{|a, b, c, \ldots|\}$ the multiset consisting of $a \Gamma$ $b \Gamma c \Gamma \ldots$ and $\uplus$ for multiset union. The notation $\langle a, b\rangle$ denotes an unordered pair $\Gamma$ which is the same as $\langle b, a\rangle$.

The unifiability problem in any language $\lambda_{\alpha}^{\beta}$ is the following:

INPUT: a finite multiset $M$ of unordered pairs of simply-typed terms in $\beta$-normal form $\left\{\left|\left\langle u_{i}, v_{i}\right\rangle\right| 1 \leq\right.$ $i \leq n \mid\}$ in $\lambda_{\alpha}^{\beta}$.

QUESTION: is there a well-typed substitution $\sigma$ such that $u_{i} \sigma=\beta \eta v_{i}$ for every $i \Gamma 1 \leq i \leq n$ ?

This problem is so close to unifiability in the simple theory of types (just ignore the levels) that a variant of Huet's procedure [Hue75] $\Gamma$ augmented with level checks $\Gamma$ will solve the problem. The idea is simple: if $\sigma$ is a ramified unifier of $M$ T then $u_{i} \sigma=\beta \eta v_{i}$ for every $i \Gamma 1 \leq i \leq n \Gamma$ so $\sigma$ is also a unifier of $M$ is simple type theory. To find such $\sigma$ 's $\Gamma$ therefore $\Gamma$ apply Huet's procedure $\Gamma$ and at specified times check that the current partial unifier has some instances that are erasures of well-typed ramified substitutions $\sigma$. The only difficulty lies in the latter: level checks need to be carefully designed. 


\subsection{Type Restrictions}

With unification in the simple theory of typesT the input $M$ could be restricted so that $u_{i}$ and $v_{i}$ had the same type for each $i \Gamma 1<i<n$; otherwise $\Gamma$ there could not be any unifier for $M$. The reason is that:

(R1) if $u$ has simple type $\epsilon$ Tthen for any well-simplytyped substitution $\sigma \Gamma u \sigma$ has type $\epsilon$ Tand

(R2) if for some well-simply-typed substitution $\sigma$ T $u \sigma$ has simple type $\epsilon$ Then $u$ has type $\epsilon$.

(R3) if $u$ has simple type $\epsilon$ and $u \Leftrightarrow \leftrightarrow^{*} \beta \eta v$ then $v$ has simple type $\epsilon$ (subject reduction) $\Gamma$ and

(R4) if $u$ has a simple type and $v$ has simple type $\epsilon$ and $u \stackrel{*}{\leftrightarrow} \beta \eta v$ Then $u$ has simple type $\epsilon$.

(R3) in fact follows from (R1) Tand (R4) follows from (R3) and the fact that each term has a unique simple type.

In the ramified theory of types $\Gamma(\mathrm{R} 1)$ still holds if we read decorated type instead of type: this is Theorem $10 \Gamma$ to be proved in Section 4. HoweverT (R2) does not hold. For instance $\Gamma$ let $u$ be $x_{\tau}^{6}$ and $\sigma$ be $\left[a_{\tau}^{2} / x\right]$ Twhere $a$ is some constant. Then the types of $u$ are all those that are at least $\tau / 6$ in the $\sqsubset \times \leq$ ordering on decorated types $\Gamma$ while those of $u \sigma$ are those of $a_{\tau}^{2}$ Tnamely all types that are at least $\tau / 2$. In particular $\Gamma u \sigma$ has types $\tau / 2 \Gamma \tau / 3 \Gamma \tau / 4 \Gamma \tau / 5 \Gamma$ which are not types of $u$.

SoT we cannot restrict ourselves to pairs $\left\langle u_{i}, v_{i}\right\rangle$ having the same decorated types. HoweverTlet's say that two decorated types $\tau / \ell$ and $\tau^{\prime} / \ell^{\prime}$ are compatible if and only if $E(\tau)=E\left(\tau^{\prime}\right)$. Then we can assume that the input to the unification problem consists of pairs $\left\langle u_{i}, v_{i}\right\rangle$ of typed terms with compatible types. Otherwise $\Gamma$ the unification problem trivially has no solution.

\subsection{Huet's Procedure}

We recall how Huet's procedure works. There is nothing new in this section $\Gamma$ as it is but a summary of [SG89].

Assume that all the terms that we use are simply typed. Huet's procedure not only tests for unifiability but returns a complete set of preunifiers $\Gamma$ in a sense which we shall recall shortly.

FirstTwe only use terms in $\beta$-normal form. This is possible because all terms are terminating. We shall write $u \downarrow$ for the unique $\beta$-normal form of the term $u$ Tand similarly for multisets of pairs of termsTetc.

These normal forms are of the form $\lambda x_{1} \cdot \ldots \cdot \lambda x_{m}$. $a u_{1} \ldots u_{n} \Gamma$ where $m \geq 0 \Gamma n \geq 0 \Gamma a$ is a variable or a constant called the head of the term Tand $u_{1} \Gamma \ldots \Gamma u_{n}$ are terms in $\beta$-normal form. For brevity as $\lambda \overline{x_{m}} \cdot a \overline{u_{n}}$. If $a$ is a constant or a bound variable $x_{i} \Gamma 1 \leq i \leq m \Gamma$ then we say that the term is rigid; it is flexible otherwise.

The $\eta$-expanded form $\eta[u]$ of a term $u$ is defined as follows: if $u=\lambda \overline{x_{m}} \cdot a \overline{u_{n}}$ and $u$ has simple type $\epsilon_{1} \rightarrow \ldots \rightarrow \epsilon_{m} \rightarrow \epsilon_{m+1} \rightarrow \ldots \rightarrow \epsilon_{m+k} \rightarrow b$ Гwhere $k \geq 0$ and $b$ is a base type $\Gamma$ then $\eta[u]$ equals:

$$
\begin{gathered}
\lambda x_{1} \cdot \ldots \cdot \lambda x_{m} \cdot \lambda x_{m+1} \epsilon_{\epsilon_{m+1}} \ldots \cdot \lambda x_{m+k_{\epsilon_{m+k}}} \cdot \\
\quad a\left(\eta\left[u_{1}\right]\right) \ldots\left(\eta\left[u_{n}\right]\right)\left(\eta\left[x_{m+1}\right]\right) \ldots\left(\eta\left[x_{m+k}\right]\right)
\end{gathered}
$$

Then $u_{i} \sigma=\beta \eta \quad v_{i} \sigma$ if and only if $\eta\left[u_{i}\right] \eta[\sigma]=\beta$ $\eta\left[v_{i}\right] \eta[\sigma]$ Twhere $\eta[\sigma]$ is the substitution mapping each $x \in \operatorname{dom} \sigma$ to $\eta[x \sigma]$; i.e. $\Gamma$ we only need compare terms by $\beta$-equality. Moreover $\Gamma$ we may look for unifiers $\sigma$ that are idempotent $\Gamma$ i.e. such that $\mathrm{FVC}(x \sigma) \cap$ dom $\sigma=\emptyset$ for every $x \in \operatorname{dom} \sigma$ Tand normalized $\Gamma$ i.e. $x \sigma$ is an $\eta$-expanded form for every $x \in \operatorname{dom} \sigma$. This is Section 2 of [SG89].

The unification procedure works by stepwise transformation of systems STi.e. of multisets of unordered pairs of terms with the same simple types. A pair $\langle\eta[x], \eta[s]\rangle$ is in solved form in $S$ if and only if the only occurrence of $x$ in $S$ is in the $\eta[x]$ component of the given pair. $S$ is itself solved if and only if all its pairs are solved in $S$. As usual $\Gamma$ a solved system $\left\{\left|\left\langle\eta\left[x_{1}\right], \eta\left[s_{1}\right]\right\rangle, \ldots,\left\langle\eta\left[x_{k}\right], \eta\left[s_{k}\right]\right\rangle\right|\right\}$ defines an idempotent and normalized substitution $\left[\eta\left[s_{1}\right] / x_{1}, \ldots, \eta\left[s_{k}\right] / x_{k}\right]$.

To approximate individual bindings $\Gamma$ we need the following notion of partial bindings (see [SG89] $\Gamma$ Definition 4.8):

Definition 3 Let $\epsilon$ be a simple type, which we write $\epsilon_{1} \rightarrow \ldots \epsilon_{n} \rightarrow b$ with $b$ a base type, and $a$ be a variable or constant of simple type $\epsilon_{1}^{\prime} \rightarrow \ldots \epsilon_{m}^{\prime} \rightarrow b$.

For each $1 \leq i \leq m$, let's write $\epsilon_{i}^{\prime}$ as $\epsilon_{1}{ }^{i} \rightarrow$ $\ldots \epsilon_{p_{i}}^{\prime^{i}} \rightarrow b_{i}$, where $b_{i}$ is a base type.

The set $B(\epsilon, a)$ of partial bindings of type $\epsilon$ and head $a$ is the set of terms of the form:

$$
\begin{aligned}
& \lambda y_{1_{\epsilon_{1}}} \cdot \ldots \cdot \lambda y_{\epsilon_{\epsilon_{n}}} \cdot \\
& \quad a\left(\lambda \overline{z_{p_{1}}^{1}} \cdot H_{1} \overline{y_{n}} z_{p_{1}}^{1}\right) \ldots\left(\lambda \overline{z_{p_{m}}^{m}} \cdot H_{m} \overline{y_{n}} \overline{z_{p_{m}}^{m}}\right)
\end{aligned}
$$

where:

1. for every $1 \leq i \leq m, 1 \leq j \leq p_{i}$, $z_{j}^{i}$ is a variable $\left(z_{j}^{i}\right)_{\epsilon_{j}^{\mu / i}}$;

2. for every $1 \leq i \leq m, H_{i}$ is a variable $\left(H_{i}\right)_{\epsilon_{1} \rightarrow \ldots \epsilon_{n} \rightarrow \epsilon_{1}^{\prime \prime i} \rightarrow \ldots \epsilon_{p_{i}}^{\prime \prime i} \rightarrow b_{i}}$; 
If $u$ has type $\epsilon$, the set $B(u, a)$ of partial bindings of head a appropriate to $u$ is $B(\epsilon, a)$.

A partial binding is an imitation binding for a if a is either a function symbol or a free variable; it is a projection binding if a is one of the bound variables $y_{i}, 1 \leq i \leq n$; we let $P B_{i}(\epsilon)$ and $P B_{i}(u)$ denote $B\left(\epsilon, y_{i}\right)$ and $B\left(u, y_{i}\right)$ respectively.

Notice that any element of $B(\epsilon, a)$ has type $\epsilon$. We say that a partial binding has fresh head variables if and only if $H_{1} \Gamma \ldots \Gamma H_{m}$ are pairwise distinct and do not occur free in the context.

Finding solved forms is not required in most applicationsTand the search for them involves a highly nondeterministic procedure [SG89]. Huet's solution was to modify the notion of solved form so that flexibleflexible pairs (i.e. Tpairs $\left\langle\lambda \overline{x_{k}} \cdot F \overline{u_{m}}, \lambda \overline{x_{k}} \cdot G \overline{v_{n}}\right\rangle$ Twith $F$ and $G$ free variables) would be considered as solved. We say that a pair in $S$ is presolved in $S$ if and only if it is either solved or consists of two flexible terms. A system $S$ is in presolved form if and only if it consists in presolved pairs.

The trick is that any flexible-flexible pair is always unifiable: for each simple type $\epsilon$ Twhich we write $\epsilon_{1} \rightarrow$ $\ldots \epsilon_{n} \rightarrow b$ with $b$ a base type $l$ let $\hat{e}_{\epsilon}$ be the term $\lambda x_{1_{\epsilon}}$. $\ldots \lambda x_{n \epsilon_{n}} \cdot v_{b} \Gamma$ where $v_{b}$ is a distinguished variable that will never be used in any other term. Letting $\zeta$ be the substitution mapping each variable of type $\epsilon$ to $\hat{e}_{\epsilon}$ Tand letting $\sigma$ be the substitution corresponding to the non-flexible-flexible pairs in $S$ T then $\sigma \zeta$ is always a unifier of $S \Gamma$ hence of $M$. Such a $\sigma$ is called the preunifier associated with $S$.

Gallier and Snyder's rules for preunification are those of Figure 2. These rules are sound (in the sense that all presolved forms denote preunifiers) and complete (in that every unifier is an instance of some preunifier derived from the rules). Moreover TGallier and Snyder show that (Delete) $($ (Decomp) and (Bind) can be applied eagerly (in particularTright after (Imitate) or (Project)). AndTas far as the flexible-rigid pair on which to apply (Imitate) or (Project) is concerned $\Gamma$ there is no non-determinism associated to its choice $\Gamma$ and we can commit ourselves to any pair we please. The restriction " $F$ free in $S$ " in (Bind) is ours $\Gamma$ as well as " $F$ unsolved" in (Imitate) and (Project); this preserves soundness and completeness $\Gamma$ and is intended to prevent this rule from being applied indefinitely in the ramified case.

\subsection{Introducing Ramification}

What do we have to do to adapt this procedure to ramified type theory? BasicallyTwe have to check all the constraints between levels that we have left out by reasoning in the simple theory of types.

The first thing is Tgiven a system $S$ in solved form describing a unifier $\sigma \Gamma$ to check whether there is a normalized instance $\left(\sigma \sigma^{\prime}\right) \downarrow$ of $\sigma$ that is a welltyped ramified substitution $\Gamma$ at least when restricted to $\mathrm{FVC}(S)$ and with proper levels annotating types and all new free variables.

We shall attack this problem by showing that it reduces to a simpler subproblem (the reduction is the content of the technical Lemma 13 to come). The simpler problem is: can we put back levels on type arrows and free variables of a term Tor of several terms $\Gamma$ so that it becomes well-typed in the ramified sense? It will turn out that this is a rather simple problem $\Gamma$ which we shall solve by graph-theoretic techniques in Section 5.

Once we have overcome these small hurdles it will be clear that ramified higher-order unification is not much more complicated that unification in the simple theory of types. In fact Tin terms of algorithmic complexity is is much simpler Tat least in the case where all levels are integer: indeed $\Gamma$ the main claim of this paper is that it is decidable.

The intuitive reason why is the following. Assume that $\sigma$ is a unifier. In rules (Imitate) and (Project) Tthe partial binding $t$ is of the general form $\lambda \overline{y_{n}}$. $a\left(\overline{\lambda \overline{z_{p_{m}}} \cdot H_{m} \overline{y_{n}} \overline{z_{p_{m}}}}\right) \Gamma$ to take a condensed notation due to Gallier and Snyder. Now Tmodulo $\sigma$ The level of $F$ should be that of $t$ Twhich by ( $A b s)$ should be at

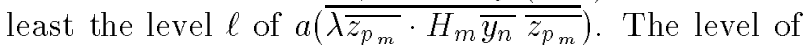
$a$ itself should then be $\ell$ by rule $(A p p)$ Tbut it can only apply to objects of strictly lower levels $\ell_{i} \Gamma 1 \leq i \leq n$ (the type of $a$ is of the form $\tau_{1} \stackrel{\ell_{1}}{\rightarrow} \ldots \tau_{m} \stackrel{\ell_{m}}{\rightarrow} \tau / \ell \Gamma$ with $\left.\ell \geq \max \left(\ell_{1}+1, \ldots, \ell_{m}+1, l(\tau)\right)\right)$. So the level of $F$ is strictly more than that of $\lambda \overline{z_{p_{i}}} \cdot H_{i} \overline{y_{m}} \overline{z_{p_{i}}}$ Tin particular than that of $H_{i}$ Tfor each $i$.

Now in any derivation $M=S_{0} \rightarrow S_{1} \rightarrow \ldots \rightarrow$ $S_{m} \rightarrow \ldots$ following the rules of Figure $2 \Gamma$ the only rules that create new variables are the above twoTand they must create variables of lower and lower ordinal levels. Therefore $\Gamma$ if we keep track of levels of free variables as above $\Gamma$ these rules can be invoked only finitely many times in such a derivation. And since (Delete) $Г($ Decomp) and (Bind) terminateTany derivation from $M$ must terminate.

As it stands This informal argument is not entirely correct Tbut it gives the rough idea. We shall formalize it in Section 6.

When the set of levels is $\omega$ or smaller $\Gamma$ it follows by König's Lemma that the whole modified procedure terminates; for the language $\lambda_{\omega}^{\omega} \Gamma$ for instance $\Gamma$ whenever we know that the level of $F$ should not ex- 


\begin{tabular}{|c|c|}
\hline (Delete) & $S^{\prime} \uplus\{|\langle u, u\rangle|\}$ \\
\hline (Decomp) & $\begin{array}{l}S^{\prime} \uplus\left\{\mid\left\langle\lambda \overline{x_{k}} \cdot a \overline{u_{n}}, \lambda \overline{x_{k}} \cdot a \overline{v_{n}}\right\} \Leftrightarrow S^{\prime} \uplus\left\{\left|\left\langle\lambda \overline{x_{k}} \cdot u_{1}, \lambda \overline{x_{k}} \cdot v_{1}\right\rangle, \ldots,\left\langle\lambda \overline{x_{k}} \cdot u_{n}, \lambda \overline{x_{k}} \cdot v_{n}\right\rangle\right|\right\}\right. \\
\text { where } a \text { is a constant or a bound variable } x_{i} \Gamma 1 \leq i \leq k\end{array}$ \\
\hline (Bind) & $\begin{array}{l}S^{\prime} \biguplus\left\{\left|\left\langle\lambda \overline{x_{k}} \cdot F \overline{x_{k}}, \lambda \overline{x_{k}} \cdot v\right\rangle\right|\right\} \Leftrightarrow\left(S^{\prime}\left[\lambda \overline{x_{k}} \cdot v / F\right]\right) \downarrow \biguplus\left\{\mid\left\langle\lambda \overline{x_{k}} \cdot F \overline{x_{k}}, \lambda \overline{x_{k}} \cdot v\right\rangle\right\} \\
\text { with } F \text { variable not free in } \lambda \overline{x_{k}} \cdot v \Gamma F \notin\left\{x_{1}, \ldots, x_{k}\right\} \Gamma F \text { free in } S^{\prime}\end{array}$ \\
\hline (Imitate) & $\begin{array}{l}S^{\prime} \biguplus\left\{\mid\left\langle\lambda \overline{x_{k}} \cdot F \overline{u_{n}}, \lambda \overline{x_{k}} \cdot a \overline{v_{m}}\right\rangle\right\} \Leftrightarrow S^{\prime} \uplus\left\{\left|\langle\eta[F], t\rangle,\left\langle\lambda \overline{x_{k}} \cdot F \overline{u_{n}}, \lambda \overline{x_{k}} \cdot a \overline{v_{m}}\right\rangle\right|\right\} \\
\text { with } F \text { unsolved variable other than } x_{1} \Gamma \ldots \Gamma x_{k} \Gamma a \text { constant } \Gamma \\
\text { and } t \in B(F, a) \text { with fresh head variables }\end{array}$ \\
\hline (Project) & $\begin{array}{l}S^{\prime} \uplus\left\{\left|\left\langle\lambda \overline{x_{k}} \cdot F \overline{u_{n}}, \lambda \overline{x_{k}} \cdot a \overline{v_{m}}\right\rangle\right|\right\} \Leftrightarrow S^{\prime} \uplus\left\{\left|\langle\eta[F], t\rangle,\left\langle\lambda \overline{x_{k}} \cdot F \overline{u_{n}}, \lambda \overline{x_{k}} \cdot a \overline{v_{m}}\right\rangle\right|\right\} \\
\text { with } F \text { unsolved variable other than } x_{1} \Gamma \ldots \Gamma x_{k} \Gamma a \text { constant or bound variable } \Gamma \\
t \in P B_{i}(F) \text { with fresh head variables and such that the head of } u_{i} \text { is } a\end{array}$ \\
\hline
\end{tabular}

Figure 2: Rules for higher-order preunification

ceed $\ell \Gamma$ we know that those of $H_{1} \Gamma \ldots \Gamma H_{m}$ should not exceed $\ell \Leftrightarrow 1$. Then $\Gamma$ the set of all derivations as above forms a finitely-branching tree whose branches are all finite sso by König's LemmaTthis tree must be finiteI yielding a terminating algorithm for unifiability in $\lambda_{\omega}^{\omega}$.

However $\Gamma$ for larger sets of levels $\Gamma$ this is not so easy; indeed $\Gamma$ when keeping track of levels as above $\Gamma$ whenever $\ell$ is a limit ordinal Twe cannot compute $\ell \Leftrightarrow 1 \Gamma$ and we have to guess a new bound $\ell_{i}<\ell$ on the level of $H_{i}$. There are always infinitely many such possible boundsT because the cofinality of any limit ordinal is at least $\omega$. Then Talthough all branches of the searchtree are finite $\mathrm{Tthe}$ rules that need to produce a partial binding must be replicated in an infinite-branching non-deterministic choice $\Gamma$ which invalidates the above argument. We conjecture that unification in $\lambda_{\alpha}^{\beta} \Gamma$ for $\alpha$ and $\beta$ large enough $\Gamma$ is undecidable. More precisely we conjecture that unification in $\lambda_{2}^{\omega+1}$ is undecidable.

\section{Basic Theorems}

We first study the $\sqsubset$ relationship $\Gamma$ which is entirely defined by the only rules $(R f l)$ and $(S u b)$ :

Lemma $1 \sqsubseteq$ is an order relation on ramified types. Moreover, if $\tau \sqsubseteq \tau^{\prime}$, then $l(\tau) \geq l\left(\tau^{\prime}\right)$.

Proof: Reflexivity follows by a straightforward induction on the type derivation.

Symmetry means that if $\tau \sqsubseteq \tau^{\prime}$ and $\tau^{\prime} \sqsubseteq \tau \Gamma$ then $\tau=\tau^{\prime}$. We show this by simultaneous induction on derivations of these two inequalities. If the last rule of at least one of the derivations is $(R f l)$ Tthen this is trivial. Otherwise $\Gamma$ the last rule is $(S u b)$ in each case $\Gamma$ so that $\tau=\tau_{1} \stackrel{\ell}{\rightarrow} \tau_{2} \Gamma \tau^{\prime}=\tau_{1}^{\prime} \stackrel{\ell^{\prime}}{\rightarrow} \tau_{2}^{\prime}$ with $\tau_{1}^{\prime} \sqsubseteq \tau_{1} \Gamma \tau_{2} \sqsubseteq \tau_{2}^{\prime}$ and $\ell^{\prime} \leq \ell$ on one hand $\Gamma$ and $\tau_{1} \sqsubseteq \tau_{1}^{\prime} \Gamma \tau_{2}^{\prime} \sqsubseteq \tau_{2}$ and $\ell<\ell^{\prime}$ on the other hand. By induction hypothesis we have $\tau_{1}=\tau_{1}^{\prime} \Gamma \tau_{2}=\tau_{2}^{\prime}$ Tand by symmetry of $\leq$ we have $\ell=\ell^{\prime}$. So $\tau=\tau^{\prime}$.

We go on to transitivity. Assume that we have derivations of $\tau \sqsubseteq \tau^{\prime}$ and of $\tau^{\prime} \sqsubseteq \tau^{\prime \prime}$ : we show that $\tau \sqsubseteq \tau^{\prime \prime}$ by simultaneous induction on the former derivations. This is clear if one of those derivations ends in $(R f l)$. Otherwise we have $\tau=\tau_{1} \stackrel{\ell}{\rightarrow} \tau_{2} \Gamma \tau^{\prime}=\tau_{1}^{\prime} \stackrel{\ell^{\prime}}{\rightarrow} \tau_{2}^{\prime} \Gamma$ $\tau^{\prime \prime}=\tau_{1}^{\prime \prime} \stackrel{\ell^{\prime \prime}}{\rightarrow} \tau_{2}^{\prime \prime} \Gamma$ with $\tau_{1}^{\prime} \sqsubseteq \tau_{1} \Gamma \tau_{2} \sqsubseteq \tau_{2}^{\prime}$ and $\ell^{\prime} \leq \ell$ on the one hand $\Gamma$ and $\tau_{1}^{\prime \prime} \sqsubseteq \overline{\tau_{1}^{\prime}} \Gamma \tau_{2}^{\prime} \sqsubseteq \overline{\tau_{2}^{\prime \prime}}$ and $\ell^{\prime \prime} \leq \ell^{\prime}$ on the other hand. By induction hypothesis it follows that $\tau_{1}^{\prime \prime} \sqsubset \tau_{1} \Gamma \tau_{2} \sqsubset \tau_{2}^{\prime \prime} \Gamma$ and by transitivity of $<$ we have $\ell^{\prime \prime} \leq \ell$ sso by one application of $(S u b)$ we get $\tau \sqsubseteq \tau^{\prime \prime}$.

Finally $\Gamma$ we show that $\tau \sqsubset \tau^{\prime}$ implies $l(\tau)>l\left(\tau^{\prime}\right)$ by induction on the derivation of the former. If the last rule is $(R f l) \Gamma$ this is clear. Otherwise we have $\tau=\tau_{1} \stackrel{\ell}{\rightarrow} \tau_{2}$ with $\tau_{1}^{\prime} \sqsubseteq \tau_{1} \Gamma \tau_{2} \sqsubseteq \tau_{2}^{\prime}$ and $\ell^{\prime} \leq \ell$. By induction hypothesis $\bar{l} l\left(\tau_{2}\right) \geq l\left(\tau_{2}^{\prime}\right) \Gamma$ so that $l(\tau)=$ $\max \left(\ell+1, l\left(\tau_{2}\right)\right) \geq \max \left(\ell^{\prime}+1, l\left(\tau_{2}^{\prime}\right)\right)=l\left(\tau^{\prime}\right)$.

It follows from this Lemma that we can normalize derivations by merging every sequence of instances of $(\mathrm{Cml})$ into just one instance. Since nothing prevents us from taking $\tau^{\prime}=\tau$ and $\ell^{\prime}=\ell$ in this rule $\Gamma$ we may also consider that exactly one instance of $(\mathrm{Cml})$ is used just after every application of $(\operatorname{Var}) \Gamma(A p p)$ or $(A b s)$.

Lemma 2 If the inequality $\tau \sqsubseteq \tau^{\prime}$ is derivable, then $\tau$ and $\tau^{\prime}$ are ramified types.

Proof: By structural induction on the derivation. If it ends in $(R f l) \Gamma$ then this is clear because then $\tau=$ $\tau^{\prime}=b$ for some base type $b$. If it ends in $(S u b) \Gamma$ then $\tau=\tau_{1} \stackrel{\ell}{\rightarrow} \tau_{2} \Gamma \tau^{\prime}=\tau_{1}^{\prime} \stackrel{\ell^{\prime}}{\rightarrow} \tau_{2}^{\prime}$. By induction hypothesis $\Gamma \tau_{1} \Gamma$ $\tau_{2} \Gamma \tau_{1}^{\prime} \Gamma \tau_{2}^{\prime}$ are ramified types. Moreover $\Gamma \ell^{\prime} \geq l\left(\tau_{1}^{\prime}\right) \Gamma$ so $\tau^{\prime}$ is a ramified type. And since $\tau_{1}^{\prime} \sqsubset \tau_{1} \Gamma$ we have $l\left(\tau_{1}^{\prime}\right) \geq l\left(\tau_{1}\right)$ by Lemma 1 : since $\ell \geq \ell^{\prime} \geq l\left(\tau_{1}^{\prime}\right) \Gamma$ it 
follows that $\ell \geq l\left(\tau_{1}\right) \Gamma$ so that $\tau$ is also a ramified type. $\square$

We then check that all the rules preserve the ramification constraints:

Lemma 3 For every term $u$, if $u: \tau / \ell$ is derivable, then $\tau$ is a ramified type and $\ell \geq l(\tau)$.

Proof: By induction on the derivation of $u: \tau / \ell$. If the last rule is ( $(\mathrm{ar}) \mathrm{T}$ then this follows from our assumption that variables and constants are decorated with ramified type $\tau$ and levels $\ell$ such that $\ell>l(\tau)$.

If the last rule is $(A b s) \Gamma$ then let it be as in Figure 1 . Then $\tau$ Ti.e. $\tau_{1} \stackrel{\ell_{1}}{\rightarrow} \tau_{2} \Gamma$ is a ramified type since $\tau_{1}$ and $\tau_{2}$ are (by assumption on the variable $y_{\tau_{1}}^{\ell_{1}}$ and by induction hypothesis respectively) and $\ell_{1} \geq l\left(\tau_{1}\right)$ (by assumption on the variable $x$ ). Moreover $\ell=$ $\max \left(\ell_{1}+1, \ell_{2}\right)$. By induction hypothesis $\ell_{2} \geq l\left(\tau_{2}\right)$. Therefore $\ell \geq \max \left(\ell_{1}+1, l\left(\tau_{2}\right)\right)=l\left(\tau_{1} \rightarrow \tau_{2}\right)=l(\tau)$.

If the last rule is $(A p p) \Gamma$ then let it be as in Figure 1. Then by induction hypothesis on the left premise $\Gamma \tau_{1} \stackrel{\ell_{1}}{\rightarrow} \tau_{2}$ is ramified Thence $\tau_{2}$ is ramified; and $\ell=\ell_{2} \geq l\left(\tau_{1} \stackrel{\ell_{1}}{\rightarrow} \tau_{2}\right)=\max \left(\ell_{1}+1, l\left(\tau_{2}\right)\right) \geq l\left(\tau_{2}\right)=l(\tau)$.

If the last rule is $(\mathrm{Cml}) \Gamma$ then by Lemma $1 l(\tau) \geq$ $l\left(\tau^{\prime}\right)$ and by induction hypothesis $\ell \geq l(\tau)$. Since $\ell^{\prime} \geq$ $\ell \Gamma i t$ follows by transitivity that $\ell^{\prime} \geq l\left(\tau^{\prime}\right)$. Moreover by Lemma $2 \tau^{\prime}$ is a ramified type Thence the claim. $\square$

\subsection{Normal Derivations}

This type system may be simplified as follows:

Definition 4 Let $\left(\mathrm{Var}^{\prime}\right)$ be the following rule:

$$
\frac{\tau \sqsubseteq \tau^{\prime} \quad \ell \leq \ell^{\prime}}{x_{\tau}^{\ell}: \tau^{\prime} / \ell^{\prime}}
$$

Call a normal type derivation any derivation of a type judgement using only rules $\left(V_{a r}^{\prime}\right),(A p p),(A b s)$, $(R f l)$ and $(S u b)$.

Lemma 4 Let $\pi$ be a normal derivation of $u: \tau_{2} / \ell_{2}$. For any ramified type $\tau_{1}^{\prime}$ and level $\ell_{1}^{\prime}$ such that $\tau_{1}^{\prime} \sqsubseteq$ $\tau_{1}, \ell_{1}^{\prime} \leq \ell_{1}$ and $\ell_{1}^{\prime} \geq l\left(\tau_{1}^{\prime}\right)$, there is a normal derivation of $u\left[y_{\tau_{1}^{\prime}}^{\ell_{1}^{\prime}} / x_{\tau_{1}}^{\ell_{1}}\right]: \tau_{2} / \ell_{2}$, where $y$ is not free in $u$.

Proof: By structural induction on $u$. If $u$ is $x_{\tau_{1}}^{\ell_{1}} \Gamma$ then $\pi$ is:

$$
\frac{\tau_{1} \sqsubseteq \tau_{2} \quad \ell_{1} \leq \ell_{2}}{x: \tau_{2} / \ell_{2}}\left(\operatorname{Var}^{\prime}\right)
$$

By the transitivity of $\sqsubseteq$ (Lemma 1 ) and of $\leq$ we have $\tau_{1}^{\prime} \sqsubseteq \tau_{2}$ and $\ell_{1}^{\prime} \leq \ell_{2} \Gamma$ so that $y_{\tau_{1}^{\prime}}^{\ell_{1}^{\prime}}: \tau_{2} / \ell_{2}$ is derivable by $\left(V a r^{\prime}\right)$ as well.
If $u$ is another variable $\Gamma$ then $u[y / x]=u$ and the claim is trivial.

If $u$ is an application $v w$ Tthen the last rule of $\pi$ was (App) Гand $\pi$ was of the form:

$$
\frac{\vdots: \tau_{3} \stackrel{\ell_{3}}{\rightarrow} \tau_{2} / \ell_{2} \quad w: \tau_{3} / \ell_{3}}{v w: \tau_{2} / \ell_{2}}(A p p)
$$

By induction hypothesis $v\left[y_{\tau_{1}^{\prime}}^{\ell_{1}^{\prime}} / x_{\tau_{1}}^{\ell_{1}}\right]: \tau_{3} \stackrel{\ell_{3}}{\rightarrow} \tau_{2} / \ell_{2}$ and $w\left[y_{\tau_{1}^{\prime}}^{\ell_{1}^{\prime}} / x_{\tau_{1}}^{\ell_{1}}\right]: \tau_{3} / \ell_{3}$ have normal derivations. Adding an instance of $(A p p)$ to the latter two yields a normal derivation of $(v w)\left[y_{\tau_{1}^{\prime}}^{\ell_{1}^{\prime}} / x_{\tau_{1}}^{\ell_{1}}\right]: \tau_{2} / \ell_{2}$.

If $u$ is an abstraction $\lambda z_{\epsilon} \cdot v$ Then $\pi$ is of the form:

$$
\frac{v\left[z_{\tau_{3}^{\prime}}^{\prime \ell_{3}} / z\right]: \tau_{4} / \ell_{4} \quad \epsilon=E\left(\tau_{3}\right) \quad z^{\prime} \notin \mathrm{FVC}(v)}{\lambda z_{f} \cdot v: \tau_{3} \stackrel{\ell_{3}}{\rightarrow} \tau_{4} / \max \left(\ell_{3}+1, \ell_{4}\right)}(A b s)
$$

where $\tau_{2}=\tau_{3} \stackrel{\ell_{3}}{\rightarrow} \tau_{4}$ and $\ell_{2}=\max \left(\ell_{3}+1, \ell_{4}\right)$. By induction hypothesis $v\left[z_{\tau_{3}}^{\prime \ell_{3}} / z\right]\left[y_{\tau_{1}^{\prime}}^{\ell_{1}^{\prime}} / x_{\tau_{1}}^{\ell_{1}}\right]: \tau_{4} / \ell_{4}$ has a normal derivation. By the variable naming convention $x \neq z \Gamma y \neq z$; and we can always transform the normal derivation above so as to ensure that $z^{\prime}$ is not equal to either $x$ or $y$. Then the term above is also $v\left[y_{\tau_{1}^{\prime}}^{\ell_{1}^{\prime}} / x_{\tau_{1}}^{\ell_{1}}\right]\left[z^{\prime \tau_{3}} / z\right] \Gamma$ and we can prolong the latter normal derivation by appending $(A b s)$ to get a normal derivation of $\left(\lambda z_{\epsilon} \cdot v\right)\left[y_{\tau_{1}^{\prime}}^{\ell_{1}^{\prime}} / x_{\tau_{1}}^{\ell_{1}}\right]: \tau_{3} \stackrel{\ell_{3}}{\rightarrow} \tau_{4} / \max \left(\ell_{3}+1, \ell_{4}\right)$.

Lemma 5 Let $\pi$ be a normal derivation of $s: \tau / \ell$. For any $\tau^{\prime}$ and $\ell^{\prime}$ with $\tau \sqsubset \tau^{\prime}$ and $\ell \leq \ell^{\prime}$, there is a normal derivation of $s: \tau^{\prime} / \ell^{\prime}$.

Proof: By induction on $\pi$ (or $s$ ). If $\pi$ ends in $\left(\mathrm{Var}^{\prime}\right)$ Tthen this follows from the transitivity of $\sqsubseteq$ and $\leq$. If $\pi$ ends in (App) Tthen this follows by induction hypothesis on the left premise.

If $\pi$ ends in $(A b s) \Gamma$ then let it be as in Figure 1: (1) $\tau=\tau_{1} \stackrel{\ell_{1}}{\rightarrow} \tau_{2}$ and (2) $\ell=\max \left(\ell_{1}+1, \ell_{2}\right)$. Since $\tau \sqsubseteq \tau^{\prime} \Gamma$ it must be the case that $(3) \tau^{\prime}=\tau_{1}^{\prime} \stackrel{\ell_{1}^{\prime}}{\rightarrow} \tau_{2}^{\prime} \Gamma$ with (4) $\tau_{1}^{\prime} \sqsubseteq \tau_{1} \Gamma(5) \tau_{2} \sqsubseteq \tau_{2}^{\prime} \Gamma(6) \ell_{1}^{\prime} \leq \ell_{1}$ and $(7)$ $l\left(\tau_{1}^{\prime}\right) \leq \ell_{1}^{\prime}$. Since $\ell^{\prime} \geq \ell$ and by (2) Twe must have (8) $\ell^{\prime} \geq \ell_{2}$. By induction hypothesis on the premise using (5) and (8) $\Gamma$ there is a normal derivation of $u\left[y_{\tau_{1}}^{\ell_{1}} / x\right]$ : $\tau_{2}^{\prime} / \ell^{\prime}$. By Lemma 4 using the fact that $\tau_{1}^{\prime}$ is a ramified type $\Gamma$ and using (4) $\Gamma(6)$ and (7) $\Gamma$ there is a normal derivation of $u\left[y_{\tau_{1}}^{\ell_{1}} / x\right]\left[y_{\tau_{1}^{\prime}}^{\prime \ell_{1}^{\prime}} / y\right]: \tau_{2}^{\prime} / \ell^{\prime}$ for any $y^{\prime}$ not free in $u\left[y_{\tau_{1}}^{\ell_{1}} / x\right]$. Choose $y^{\prime}$ not free in $u$ and different 
from $y$. As $y$ is not free in $u$ Tit follows that we have a normal derivation of $u\left[y_{\tau_{1}^{\prime}}^{\ell_{1}^{\prime}} / x\right]: \tau_{2}^{\prime} / \ell^{\prime}$. ThenTby (4) $E\left(\tau_{1}\right)=E\left(\tau_{1}^{\prime}\right)=\epsilon \Gamma$ so we can apply $(A b s)$ and get a normal derivation of $\lambda x_{\epsilon} \cdot u: \tau_{1}^{\prime} \stackrel{\ell_{1}^{\prime}}{\rightarrow} \tau_{2}^{\prime} / \max \left(\ell_{1}^{\prime}+1, \ell^{\prime}\right)$. Since $\ell^{\prime} \geq \ell$ and by (2) $\Gamma$ we have $\ell^{\prime} \geq \ell_{1}+1 \Gamma$ whence $\ell^{\prime} \geq \ell_{1}^{\prime}+1$ by $(6) \Gamma$ so $\max \left(\ell_{1}^{\prime}+1, \ell^{\prime}\right)=\ell^{\prime}$. Moreover $\tau_{1}^{\prime} \stackrel{\ell_{1}^{\prime}}{\rightarrow} \tau_{2}^{\prime}$ is just $\tau^{\prime}$ by (3) $\Gamma$ so that we have a normal derivation of $\lambda x_{\epsilon} \cdot u: \tau^{\prime} / \ell^{\prime}$.

Theorem 6 (Normalization) A typing judgement $u: \tau / \ell$ has a derivation in the system of Figure 1 if and only if it has a normal derivation.

Proof: If: any normal derivation can be trivially rewritten into a derivation in the system of Figure 1 by replacing each instance of $\left(V a r^{\prime}\right)$ into the appropriate instances of ( $\mathrm{Var})$ and $(\mathrm{Cml})$.

Only if: this is proved by structural induction on a derivation $\pi$. If $\pi$ ends in ( $V$ ar) and concludes $x_{\tau}^{\ell}: \tau / \ell \Gamma$ then we replace it by:

$$
\frac{\tau \sqsubseteq \tau \quad \ell \leq \ell}{x_{\tau}^{\ell}: \tau / \ell}\left(\operatorname{Var}^{\prime}\right)
$$

Indeed $\Gamma$ because $\Gamma, x: \tau / \ell$ is a context $\Gamma \tau$ is a ramified type $\Gamma$ and therefore $\tau \sqsubseteq \tau$ is derivable by Lemma 1 .

If $\pi$ ends in ( $A p p$ ) or in ( $A b s$ ) then by induction hypothesis we can transform the subderivations whose conclusions are the premises of the rules into normal derivations. This transforms $\pi$ into a normal derivation.

Finally $\pi$ if $\pi$ ends in $(\mathrm{Cml})$ Tthen it has the form:

$$
\frac{u: \tau / \ell \tau \sqsubseteq \tau^{\prime} \quad \ell \leq \ell^{\prime}}{u: \tau^{\prime} / \ell^{\prime}}
$$

By induction hypothesis $\Gamma$ there is a normal proof $\pi^{\prime \prime}$ of $u: \tau / \ell$. By Lemma $2 \Gamma \tau^{\prime}$ is a ramified type $\Gamma$ so by Lemma 5 There is a normal derivation of $u: \tau^{\prime} / \ell^{\prime}$.

One property that we have in the simple theory of types and which fails here is the unicity of types. NamelyTevery typable term has many different types $\Gamma$ because of the subtyping rule $(S u b)$. Because of this same rule $\Gamma$ the set of types of a given typable term is an upper $\sqsubseteq$-ideal. This will complicate a bit our analysis of unification.

\subsection{The Vicious Circle Principle}

One of the arguments that Russell proposed in favor of ramification was that it disallowed so-called vicious circles ("whatever involves all of a collection must not be one of the collection"). Theorem 8 below gives the precise sense in which our system implements Russell's vicious circle principle.

Lemma 7 Let $x$ be a variable or a constant. Let $s$ be a term where $x_{\tau}^{\ell}$ occurs free, and assume that $s: \tau^{\prime} / \ell^{\prime}$ is derivable in the system of Figure 1 . Then $\ell^{\prime} \geq \ell$.

Proof: By induction on the derivation. If the last rule is $($ Var $) \Gamma$ this is obvious. If it is $(\mathrm{Cml}) \Gamma$ this follows directly from the induction hypothesis.

If the last rule is $(A p p)$ Tthen $s=u v$ and $\ell^{\prime}=\ell_{2}$. If $x$ is free in $u$ Tthen by the induction hypothesis on the left premise $\Gamma \ell^{\prime} \geq \ell$; otherwise $\Gamma x$ must be free in $v \Gamma$ so that by induction hypothesis in the right premise $\Gamma$ $\ell_{1} \geq \ell$. But by Lemma 3 on the left premise $\Gamma \ell_{2} \geq$ $l\left(\tau_{1} \stackrel{\ell_{1}}{\rightarrow} \tau_{2}\right)=\max \left(\ell_{1}+1, l\left(\tau_{2}\right)\right) \geq \ell_{1}+1>\ell_{1} \geq \ell \Gamma$ hence $\ell^{\prime}>\ell$.

If the last rule is $(A b s) \Gamma$ then $s=\lambda x_{\epsilon} \cdot u$ and $\ell^{\prime}=$ $\max \left(\ell_{1}+1, \ell_{2}\right) \Gamma$ where by induction hypothesis $\ell_{2} \geq \ell$. So $\ell^{\prime} \geq \ell_{2}$.

Theorem 8 Let $u_{1}, \ldots, u_{n}$ be terms, $n \geq 1$, and assume that the variable or constant $x$ occurs free in some $u_{i}, 1 \leq i \leq n$. Then $x u_{1} \ldots u_{n}$ is not typable in the system of Figure 1.

Proof: Assume that $x u_{1} \ldots u_{n}$ is typable. By Theorem 6 it has a normal derivation. Furthermore $x$ must be of the form $x^{\ell} \tau_{1} \tau_{\ell_{n}}$ Twith in particular (1) $\ell \geq \max \left(\ell_{1}+1, \ldots, \ell_{n}+1, l(\tau)\right)$.

Without loss of generality $\Gamma$ we may consider that $x$ is free in $u_{n}$. Consider a normal derivation of a decorated type for $x u_{1} \ldots u_{n}$ :

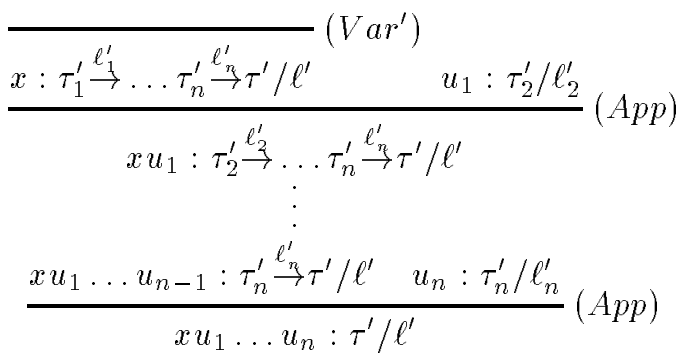

where in particular $(2) \ell_{i}^{\prime} \leq \ell_{i}$ for every $1 \leq i \leq n$. By Lemma 7 and since $x$ is free in $u_{n} \Gamma \ell_{n}^{\prime} \geq \ell$. By (2) with $i=n \Gamma$ it follows that $\ell_{n} \geq \ell$. By (1) $\ell \geq \ell_{n}+1 \Gamma$ hence $\ell \geq \ell+1$ Twhich is impossible. $\square$

So $\Gamma$ if $x$ is a $n$-ary predicate variable $\Gamma$ we cannot express the fact that it must hold of some $n$-tuple of individuals whose definitions involve $x$ itself. This is how ramification prohibits vicious circles. 


\subsection{Reduction}

Let's now return to more mundane $\Gamma$ basic propertiesTand in particular let's examine how term typings evolve through reduction. FirstTramification behaves well under substitution:

Lemma 9 If $u: \tau^{\prime} / \ell^{\prime}$ and $v: \tau / \ell$ are derivable, then so is $u\left[v / x_{\tau}^{\ell}\right]: \tau^{\prime} / \ell^{\prime}$.

Proof: By induction on the derivation of $u: \tau^{\prime} / \ell^{\prime}$. If $u=x \Gamma$ then this is obvious. Otherwise $\Gamma$ the last rule in the derivation is $(A p p) \Gamma(A b s) \Gamma$ or $(C \mathrm{Cl}) \Gamma$ and the claim follows straightforwardly from the induction hypothesis (and the fact that $\Gamma$ thanks to the variable naming convention in the case of $(A b s)$ Twe have $(\lambda y$. $u)[v / x]=\lambda y \cdot u[v / x])$.

Theorem 10 (Subject Reduction) If $s: \tau / \ell$ is derivable and $s \stackrel{*}{\leftrightarrow} \beta$ (resp. $s \stackrel{*}{\leftrightarrow} \beta \eta t$ ), then $t: \tau / \ell$ is derivable.

Proof: By induction on the length of a normal derivation. It is enough to prove the claim for the two cases $s \Leftrightarrow \rightarrow_{\star} t$ Twhere $\star$ is either $\beta$ or $\eta$. Take $s=\mathcal{C}\left[s^{\prime}\right] \Gamma$ $t=\mathcal{C}\left[t^{\prime}\right]$ Twhere $s^{\prime}$ is the redex and $t^{\prime}$ is its contractum. We prove the claim by induction on $\mathcal{C}$. The induction cases $\left(\mathcal{C}\right.$ of the form $\mathcal{C}^{\prime} w \Gamma w \mathcal{C}^{\prime}$ or $\left.\lambda x \cdot \mathcal{C}^{\prime}\right)$ are triviall and it remains to prove the base case $\Gamma$ namely when $s$ is itself the redex.

If $s$ is a $\beta$-redex $\left(\lambda x_{\epsilon} \cdot u\right) v \Gamma$ then $t=u[v / x]$. Look at the normal derivation of $s: \tau / \ell$ :

$$
\begin{gathered}
\frac{u\left[y_{\tau_{1}}^{\ell_{1}} / x\right]: \tau / \ell}{\lambda x_{\epsilon} \cdot u: \tau_{1} \stackrel{\ell_{1}}{\rightarrow} \tau / \ell}(A b s) \quad \vdots \\
\left(\lambda x_{\epsilon} \cdot u\right) v: \tau / \ell
\end{gathered}
$$

where $\epsilon=E\left(\tau_{1}\right)$. By Lemma $9 \Gamma u\left[y_{\tau_{1}}^{\ell_{1}} / x\right]\left[v / y_{\tau_{1}}^{\ell_{1}}\right]: \tau / \ell$ is derivable. Since $y$ is not free in $u \Gamma$ the latter term is $u[v / x] \Gamma$ whence the claim.

If $s$ is an $\eta$-redex $\lambda x_{\epsilon} \cdot u x$ Twith $x$ not free in $u$ Tthen necessarily the derivation of $s: \tau / \ell$ ends in:

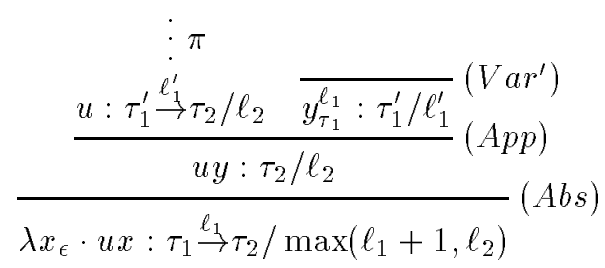

where (1) $\tau_{1} \sqsubseteq \tau_{1}^{\prime} \Gamma(2) \ell_{1} \leq \ell_{1}^{\prime}$ (rule $\left.\left(\operatorname{Var}^{\prime}\right)\right) \Gamma(3)$ $\epsilon=E\left(\tau_{1}\right)$ (rule $(A b s)$ ) and (4) $\tau=\tau_{1} \stackrel{\ell_{1}}{\rightarrow} \tau_{2} \Gamma(5) \ell=$ $\max \left(\ell_{1}+1, \ell_{2}\right)$. Moreover $(6) \ell_{1} \geq l\left(\tau_{1}\right)$ because of the assumption on the decoration of the variable $y$. By (1) $\Gamma(2)$ and (6) it follows that $\tau_{1}^{\prime} \ell_{1}^{\prime} \tau_{2} \sqsubseteq \tau_{1} \stackrel{\ell_{1}}{\rightarrow} \tau_{2}$; since $\ell_{2} \leq \max \left(\ell_{1}+1, \ell_{2}\right)$ as well $\Gamma$ we can build the derivation:

$$
\frac{\vdots: \pi}{u: \tau_{1}^{\prime} \stackrel{\ell_{1}^{\prime}}{\rightarrow} \tau_{2} / \ell_{2}}(C m l)
$$

which proves the claim.

Subject reduction is important $\Gamma$ for without it $\beta$ reduction or $\beta \eta$-reduction would be a meaningless concept within the ramified typed universe. In particularT the Church-Rosser property of the untyped $\lambda$-calculus transfers to the ramified typed $\lambda$-calculus just because subject reduction works (and because of $\alpha$-conversion). Also Tit will be important in unification $\Gamma$ because applying a substitution to a term is then followed by a normalization phase $\Gamma$ which by Theorem 10 will preserve well-typedness.

Equally important is the fact that $\Gamma$ in some sense $\Gamma$ the ramified type theory is a subtheory of the simple type theory $\mathrm{Ta}$ fact that we have used in the informal presentation of Sections 3.2 and 3.3 :

Lemma 11 If $u: \tau / \ell$ is derivable in the system of Figure 1, then $u: E(\tau)$ is derivable in the simple theory of types.

Proof: To be precise Tby the simple theory of types we mean the set of rules:

$$
\begin{gathered}
\frac{x_{\tau}^{\ell}: E(\tau)}{\text { Var } \left.^{*}\right)} \\
\frac{u: \epsilon_{1} \rightarrow \epsilon_{2} \quad v: \epsilon_{1}}{u v: \epsilon_{2}}\left(A p p^{*}\right) \\
\frac{u: \epsilon_{2}}{\lambda x_{\epsilon_{1}} \cdot u: \epsilon_{1} \rightarrow \epsilon_{2}}\left(A b s^{*}\right)
\end{gathered}
$$

i.e. T we keep levels on variables $\Gamma$ although they are useless.

The claim is then a trivial induction on the derivation: $(\operatorname{Var}) \Gamma(A p p)$ and $(A b s)$ rules translate to $\left(V a r^{*}\right) \Gamma\left(A p p^{*}\right)$ and $\left(A b s^{*}\right)$ respectivelyTwhile $(\mathrm{Cml})$ steps are erased. Indeed $\Gamma$ whenever $\tau \sqsubseteq \tau^{\prime} \Gamma$ we have $E(\tau)=E\left(\tau^{\prime}\right)$ : this is an easy induction on the derivation of the inequality.

Corollary 12 The ramified theory of types is convergent: every $\beta$ (resp. $\beta \eta$ ) reduction terminates to a unique normal form. 
Proof: Every derivation in the ramified calculus is also a derivation in the simply-typed calculus. (In particularTany $\alpha$-conversion step in the former is an $\alpha$-conversion step in the latter.) So the ramified calculus is terminating.

ConverselyClet $u$ be a term of decorated type $\tau / \ell$ that reduces to $v$ and $w$ along different reduction paths. Because the pure $\lambda$-calculus is confluent $\Gamma$ the simply-typed calculus is [Bar84]; now $v$ and $w$ have types $E(\tau)$ in the simple theory of types by Lemma 11 applied to $u$ and Theorem 10 . So $v$ and $w$ have a common reduct $s$ in the simple theory of types. But the reductions from $v$ to $s$ and from $w$ to $s$ are also reductions in the ramified calculus: this establishes the fact that the ramified calculus is confluent Thence Church-Rosser.

FinallyTany rewrite system that is both terminating and Church-Rosser is convergent.

Recall that we write $u \downarrow_{\beta}$ Tor simply $u \downarrow$ Tthe unique $\beta$-normal form of $u$.

\subsection{Can a Term be Instantiated to a Well-Typed One?}

There is a kind of converse of Theorem 10 Tor a kind of analogue of remark (R4) at the beginning of Section 3.1. The goal is to establish Theorem 15 Twhich states a necessary and sufficient condition for a term to have well-typed ramified instances.

Lemma 13 For every simple type $\epsilon$, writen uniquely as $\epsilon_{1} \rightarrow \ldots \epsilon_{n} \rightarrow b$ with $b$ a base type, let $\hat{e}_{f}$ be the term $\lambda x_{1 \epsilon_{1}} \cdot \ldots \lambda x_{n_{n}} \cdot v_{b}^{0}$, where $v_{b}^{0}$ is a distinguished variable of type $b$ and level 0 that will never be used in any other term.

We let $\zeta$ be the substitution mapping each variable of type $\tau$ (and arbitrary level) to $\hat{e}_{E(\tau)}$. Then:

1. $\zeta$ is a well-typed substitution;

2. for every $\beta$-normal term $t$, the $\beta$-normal form $(t \zeta) \downarrow$ exists;

3. for every $\beta$-normal simply-typed term $t$, for every well-simply-typed substitution $\sigma$, if $t \sigma \beta$-reduces to a ramified well-typed term of some decorated type $\tau / \ell$, then $(t \zeta) \downarrow: \tau / \ell$ is derivable.

Proof: 1 . Let $x_{\tau}^{\ell}$ be any variableTwith $(1) \ell \geq l(\tau) \mathrm{\Gamma}$ (2) $\tau=\tau_{1} \stackrel{\ell_{1}}{\rightarrow} \ldots \tau_{n} \stackrel{\ell_{n}}{\rightarrow} b \Gamma$ with $b$ a base type $\Gamma$ and $\epsilon=$ $E(\tau) \Gamma$ so that $x \zeta=\hat{e}_{\epsilon}$. By $n$-fold application of rule $(A b s) \Gamma \hat{e}_{\epsilon}: \tau_{1} \stackrel{\ell_{1}}{\rightarrow} \ldots \tau_{n} \stackrel{\ell_{n}}{\rightarrow} b / \max \left(\ell_{1}+1, \ldots, \ell_{n}+1,0\right)$ is derivable. By $(2) \Gamma \max \left(\ell_{1}+1, \ldots, \ell_{n}+1,0\right)=l(\tau) \Gamma$ so by (1) it is at most $\ell$ : apply $(\mathrm{Cml})$ to get a derivation of $\hat{e}_{\epsilon}: \tau / \ell$.
2. Let $W$ be $\mathrm{FVC}(t) \Gamma$ and let $\sigma_{0}$ be $\left.\zeta\right|_{W} \Gamma$ so that $\sigma_{0}$ is a regular substitution with $u \sigma_{0}=u \zeta$. Recall that $\beta$-normal forms may be characterized as those terms defined inductively as $\lambda \overline{y_{k}} \cdot a \overline{u_{m}}$ Twhere $k, m \geq 0 \Gamma$ $a$ is a variable or a constant $\Gamma$ and $u_{1} \Gamma \ldots \Gamma u_{m}$ are normal forms. (We call this the hnf-decomposition of the term.) The (unique) normal form $(t \zeta) \downarrow$ (i.e. $\Gamma$ $\left.\left(t \sigma_{0}\right) \downarrow\right)$ may then be defined by recursion on its hnfdecomposition by:

- if $a \in W \Gamma$ then $a \sigma_{0}$ is some $\hat{e}_{\epsilon} \Gamma$ with $\epsilon=\epsilon_{1} \rightarrow$ $\ldots \epsilon_{n} \rightarrow b \Gamma n \geq m \Gamma$ and $\left(t \sigma_{0}\right) \downarrow=\lambda y_{1} \cdot \ldots \cdot \lambda y_{k}$. $\lambda x_{m+1} \epsilon_{m+1} \cdot \ldots \cdot \lambda x_{n \epsilon_{n}} \cdot v_{b}^{0}$;

- otherwise $\Gamma\left(t \sigma_{0}\right) \downarrow=\lambda \overline{y_{k}} \cdot a \overline{\left(u_{m} \sigma_{0}\right) \downarrow}$.

3. Let $W \Gamma \sigma_{0}$ be as above. We prove the claim by induction on the hnf-decomposition of $t$ Wwith $t$ as in 2. Let $y_{i}$ be $y_{i_{\epsilon^{\prime}}} \Gamma$ for every $1 \leq i \leq k \Gamma$ and assume that $t \sigma \stackrel{*}{\leftrightarrow} \beta \beta$ for some typable $s$. Then:

- if $a \in W \Gamma$ then look at $s$. Whenever $s: \tau / \ell$ is derivable $\Gamma s$ has type $E(\tau)$ in the simple theory of types by Lemma 11 . So t $\sigma$ has type $E(\tau)$ in the simple theory of types (see Remark (R4) at the beginning of Section 3.1). But the simple type of $a$ is some $\epsilon$ of the form $\epsilon_{1} \rightarrow \ldots \epsilon_{n} \rightarrow b \Gamma$ $n \geq m$ Tso the simple type of $t \sigma$ must be $E(\tau)=$ $\epsilon_{1}^{\prime} \rightarrow \ldots \rightarrow \epsilon_{k}^{\prime} \rightarrow \epsilon_{m+1} \rightarrow \ldots \rightarrow \epsilon_{n} \rightarrow b$. For this to be the case $\tau$ must be of the form (1) $\tau_{1}^{\prime} \stackrel{\ell_{1}^{\prime}}{\rightarrow} \ldots \stackrel{\ell_{k-1}^{\prime}}{\rightarrow} \tau_{k}^{\prime} \stackrel{\ell_{k}^{\prime}}{\rightarrow} \tau_{m+1} \stackrel{\ell_{m+1}}{\rightarrow} \ldots \stackrel{\ell_{n}-1}{\rightarrow} \tau_{n} \stackrel{\ell_{n}}{\rightarrow} b \Gamma$ with $(2)$ $E\left(\tau_{i}^{\prime}\right)=\epsilon_{i}^{\prime} \Gamma 1 \leq i \leq k$ and $(3) E\left(\tau_{j}\right)=\epsilon_{j} \Gamma 1 \leq$ $j \leq n$. Moreover by Lemma 3 we have (4) $\ell \geq$ $l(\tau)$.

On the other hand $\Gamma\left(t \sigma_{0}\right) \downarrow=\lambda y_{1 \epsilon_{1}^{\prime}} . \ldots$. $\lambda y_{\epsilon_{k}^{\prime}} \cdot \lambda x_{m+1 \epsilon_{m+1}} \cdot \ldots \cdot \lambda x_{\epsilon_{\epsilon_{n}}} \cdot v_{b}^{0} \cdot \quad$ Applying rule $(A b s) k$ times using (2) and $n \Leftrightarrow m$ times using $(3) \Gamma$ it follows that $\left(t \sigma_{0}\right) \downarrow$ : $\tau_{1}^{\prime} \stackrel{\ell_{1}^{\prime}}{\rightarrow} \ldots \stackrel{\ell_{k}^{\prime}}{\rightarrow} \tau_{k}^{\prime} \stackrel{\ell_{k}^{\prime}}{\rightarrow} \tau_{m+1} \stackrel{\ell_{m+1}}{\rightarrow} \ldots \stackrel{\ell_{n}-1}{\rightarrow} \tau_{n} \stackrel{\ell_{n}}{\rightarrow} b / \max \left(\ell_{1}^{\prime}+\right.$ $\left.1, \ldots, \ell_{k}^{\prime}+1, \ell_{m+1}+1, \ldots, \ell_{n}+1\right)$ is derivable $\Gamma$ i.e. $\left(t \sigma_{0}\right) \downarrow: \tau / l(\tau)$ using (1). By $(\mathrm{Cml})$ and (4) Tit follows that $\left(t \sigma_{0}\right) \downarrow: \tau / \ell$ is derivable.

- If $a \notin W \Gamma$ then $t \sigma=\lambda \overline{y_{k}} \cdot a \overline{u_{m} \sigma} \Gamma$ and $a$ is a constant or a variableTso $s$ must be of the form $\lambda \overline{y_{k}} \cdot a \overline{v_{m}}$ with $u_{i} \sigma \stackrel{*}{\leftrightarrow} \beta v_{i}$ for every $1 \leq i \leq m$. MoreoverTsince $s$ is (ramified) typable $\Gamma$ so is each $v_{i}$ (with $y_{1} \Gamma \ldots \Gamma y_{k}$ replaced by suitable fresh variables of the right types and levels). By induction hypothesis $\Gamma\left(u_{i} \sigma_{0}\right) \downarrow$ can be assigned the same decorated type as $v_{i}$ for each $1 \leq i \leq m$. It follows that $\lambda \overline{y_{k}} \cdot a \overline{\left(u_{m} \sigma_{0}\right) \downarrow}$ can be given the same decorated type as $s$; but this is just $\left(t \sigma_{0}\right) \downarrow$ by construction. 
The case of the $\eta$-rule is more trivial:

Lemma 14 If $u \stackrel{*}{\Leftrightarrow} \eta, u$ is simply-typed and $v: \tau / \ell$ is derivable, then $u: \tau / \ell$.

Proof: By induction on the length of the reduction from $u$ to $v \Gamma$ it is enough to prove the claim when $u \Leftrightarrow \rightarrow_{\eta} v$. In turn $\Gamma$ this is proved by structural induction on $\mathcal{C} \Gamma$ where $u=\mathcal{C}\left[\lambda x_{\epsilon_{1}} \cdot t x\right]$ and $v=\mathcal{C}[t]$. The only non trivial case is the base case $\Gamma$ where $\mathcal{C}$ is the empty context. Then $\Gamma$ assume $t: \tau / \ell$ derivable. Because $u$ is simply-typed and by Lemma $11 \Gamma$ we must have $E(\tau)=\epsilon_{1} \rightarrow \epsilon_{2}$ for some simple type $\epsilon_{2}$. So $\tau$ must be of the form $\tau_{1} \stackrel{\ell_{1}}{\rightarrow} \tau_{2}$ Twith $\epsilon_{1}=E\left(\tau_{1}\right) \Gamma$ $\epsilon_{2}=E\left(\tau_{2}\right) \Gamma \ell_{1} \geq l\left(\tau_{1}\right)$ and $\ell \geq \max \left(\ell_{1}+1, l\left(\tau_{2}\right)\right)$. Since $x$ is not free in $t$ by assumption $\Gamma$ we can produce the following derivation:

$$
\frac{\vdots}{\frac{t y_{\tau_{1}}^{\ell_{1}}: \tau_{2}}{\lambda x_{\epsilon_{1}} \cdot t x: \tau_{1} \stackrel{\ell_{1}}{\rightarrow} \tau_{2} / \max \left(\ell_{1}+1, l\left(\tau_{2}\right)\right)}}(\mathrm{Cml})
$$

Theorem 15 Let $t$ be a $\beta$-normal simply-typed term. Call a well-simply-typed instance of $t$ any term $t \sigma$, where $\sigma$ is a well-simply-typed substitution.

Then $t$ has a well-simply-typed instance that is $\beta \eta$ equivalent to a ramified well-typed term if and only if $(t \zeta) \downarrow$ has a ramified type.

Proof: If the term $t$ has a well-simply-typed instance $t \sigma$ that is $\beta \eta$-equivalent to a well-typed ramified term $u$ Tthen by confluence $t \sigma \stackrel{*}{\leftrightarrow} \beta \eta v$ and $u \stackrel{*}{\leftrightarrow} \beta \eta$ for some term $v$. By Theorem 10 applied to $u$ Tit follows that $t$ has a well-simply-typed instance $t \sigma$ that $\beta \eta$-reduces to some ramified well-typed term. Conversely if $t \sigma \beta \eta$-reduces to some ramified well-typed term $\Gamma$ it is $\beta \eta$-equivalent to it. To prove the theorem $I$ it is therefore enough to prove the following claim: $t$ has a well-simply-typed instance that $\beta \eta$-reduces to a ramified well-typed term if and only if $(t \zeta) \downarrow$ has a ramified type.

By Lemma 131 . and 2. $(t \zeta) \downarrow$ is always welldefined and that $t \zeta$ is a well-typed instance of $t$. Recall also that $\Gamma i n$ any $\beta \eta$-reduction to normal form $\Gamma$ we may first take the $\beta$-normal form $\Gamma$ then the $\eta$-normal form of the latter (this is postponement of the $\eta$-rule [Bar84]).

If: if $(t \zeta) \downarrow$ has a ramified type $\Gamma$ then let $t \zeta$ be the desired instance: it $\beta$-reduces to a term with a ramified type $\Gamma$ namely $(t \zeta) \downarrow$ Wwhich then $\eta$-normalizes to another term with the same ramified type $\Gamma$ by Theorem 10 .

Only if: assume that there is a substitution $\sigma$ such that $t \sigma \stackrel{*}{\Leftrightarrow} \beta \eta v$ with $v$ having the ramified decorated type $\tau / \ell$. By postponement of $\eta \Gamma$ there is a term $u$ such that $t \sigma \stackrel{*}{\leftrightarrow} \beta, \quad \stackrel{*}{\leftrightarrow} \rightarrow_{\eta} v$. By subject reduction in the simply-typed case $\Gamma u$ is simply typed. By Lemma $14 \Gamma$ $u: \tau / \ell$ is therefore derivable. By Lemma $13 \Gamma(t \zeta) \downarrow$ : $\tau / \ell$ is then derivable.

Therefore $\Gamma$ the problem of finding instances of a simply-typed term modulo $\beta \eta$ that is well-typed in the ramified sense reduces to the type-checking problem in the ramified system of Figure 1.

\section{$5 \quad$ Retrieving Levels}

We wish to show that type-checking ramified terms is decidableTand in fact computationally easy. To typecheck a term Wwe wish to find a normal derivation of a decorated type for itTor to prove that none exists. We do this classically by constructing such a derivation bottom-upTbeing guided by the structure of the term. The only problem lies in rule $(A b s) \Gamma$ because there we need to guess a type $\tau_{1}$ and a level $\ell_{1}$ for the fresh variable $y$. Since we already know the shape of $\tau_{1}\left(E\left(\tau_{1}\right)\right.$ must equal $\left.\epsilon\right) \Gamma$ this is just a question of guessing the right levels to annotate the function arrows in $\tau_{1}$.

So first Twe replace every level annotating variables or type arrows by fresh level variables $\Gamma$ and express the typing constraints as systems of level constraints in Section 5.1. We shall then show that we can infer the general form of types and levels of terms in polynomial time in Section 5.2. This is the analogue of [Hin69] or of the ML type system [Mil78] without lets. We give a few examples in Section 5.3Tand propose a few improvements in Section 5.4.

\subsection{Level Variables and Constraints}

Introducing level variables demands that we change our representation of types and terms to accomodate variables:

Definition 5 Let $V$ be a coutably infinite set of socalled level variables $\mu, \nu$, etc.

The set $R T(V)$ of ramified pre-types with variables as levels is the smallest containing all base types $b$, and all expressions $\tau_{1} \stackrel{\mu}{\rightarrow} \tau_{2}$, where $\tau_{1}$ and $\tau_{2}$ are ramified pre-types and $\mu$ is in $V \cup L$.

The set $\lambda(V)$ of ramified pre-terms is the smallest containing variables $x_{\tau}^{\mu}$ where $\tau \in R T(V)$ and $\mu \in$ 
$V \cup L$, applications uv with $u \in \lambda(V)$ and $v \in \lambda(V)$, and abstractions $\lambda x_{\epsilon} \cdot u$ with $u \in \lambda(V)$ and $\epsilon \in S T$.

Notice that a pre-term with no level variables is just an ordinary term. These new objects are to be understood under assignments $\rho$ mapping level variables to actual levels in $L$. To represent sets of assignmentsI we use the following notion of constraints.

Definition $6 A$ system of level constraints $K$ is a finite set of constraints of the form $\mu \geq \nu+n$ where $\mu$ and $\nu$ are level variables and $n \in \mathbb{N}$, or $\mu \geq \ell$ or $\ell \geq \mu$ where $\mu$ is a level variable and $\ell$ is a constant level in $L$.

The domain dom $K$ of a system $K$ is the set of level variables appearing in it. A level assignment $\rho$ is said to satisfy $K$, and we write $\rho \models K$, if and only if all the inequalities gotten from constraints in $K$ by replacing variables $\mu$ by $\rho(\mu)$ are valid.

For any sentence about $R T(V)$ or $\lambda(V)$, we say that it holds under $K$ provided that for any $\rho$ satisfying $K$, the same sentence holds with all level variables replaced by their values under $\rho$.

Systems of level constraints are interesting because they express all the constraints that we shall needTand most problems on them are solvable in polynomial time. But before we can speak of polynomial timeI we must define our data representations $\Gamma$ and define measures of size. We define the size $|o|$ of an object $o$ as usual $\log |u v|=|u|+|v|+1 \Gamma\left|\lambda x_{\epsilon} \cdot u\right|=|\epsilon|+|u|+1 \Gamma$ $\left|x_{\epsilon}\right|=|\epsilon|+1 \Gamma\left|x_{\tau}^{\mu}\right|=|\tau|+1 \Gamma|b|=1 \Gamma\left|\epsilon_{1} \rightarrow \epsilon_{2}\right|=$ $\left|\epsilon_{1}\right|+\left|\epsilon_{2}\right|+1 \Gamma\left|\tau_{1} \stackrel{\mu_{1}}{\rightarrow} \tau_{2}\right|=\left|\tau_{1}\right|+\left|\tau_{2}\right|+1$.

Then Twe represent $K$ as the following graph $G(K)$ : the vertices are all variables in dom $K$ and all level constants appearing in $K \Gamma$ and the edges are $\mu \Leftrightarrow \rightarrow$ for all constraints $\mu \geq \nu+n \Gamma \mu \Leftrightarrow \stackrel{0}{\leftrightarrow} \ell$ for all constraints $\mu \geq \ell$ and $\ell \Leftrightarrow \rightarrow$ for all constraints $\ell \geq \mu$ in $K$. The labels on arrows are called weights. It will always be assumed that $G(K)$ is in fact the way that $K$ is really represented in memory 5 so that we don't have to translate back and forth between $K$ and $G(K)$ in practice. Furthermore we assume that $G(K)$ is represented in memory as an adjacency list [McH90] $\Gamma$ i.e. a list of vertices $\Gamma$ as pointers to records containing the description of the vertex (an ordinal $\Gamma$ or a special tag denoting a variable vertex) $\Gamma$ and a list of successor/weight pairs. (If there are two edges from $v_{1}$ to $v_{2} \Gamma$ with respective weights $n_{1}$ and $n_{2} \Gamma$ we only represent one with weight $\max \left(n_{1}, n_{2}\right)$; this does not change the semantics of $K$.) The size $|K|$ of $K$ is then the sum of sizes of edges (equated to the size $|n|=\max \left(1,\left[\log _{2}(n+1)\right]\right)$ of the weights labelling them Trepresented in binary) Tplus the sum of sizes of vertices in dom $K T$ where a variable vertex has size 1 .
To define the size of constant verticesT we need to make precise our system of ordinal notations. It must allow us to compute the sum of an ordinal and an integer $\Gamma$ to compare by $=$ or $\leq$ any two ordinals in polynomial time. If we use only integers as levels $\Gamma$ say in binaryTthis is trivial (the size of $\ell$ is then $|\ell|=$ $\left.\max \left(1,\left[\log _{2} \ell\right\rceil\right)\right)$. This is also certainly possible up to $\Gamma_{0}$ [Gal91] Tby using Schütte's $\psi$ function and natural ordinal sums: this yields ordinal notations where $=$ is just structural comparison and $<$ is the lexicographic path ordering. The size of an ordinal notation $\Gamma$ there $\Gamma$ is the number of signs needed to write it on paper.

The essence of our algorithms will be the computation of the set of strongly connected components of $G(K)$ [McH90]. Recall that a subgraph is strongly connected if and only if every vertex in the subgraph is reachable from any other vertex in the subgraphTand that the strongly connected components of a graph of the maximal strongly connected subgraphs. Leaving labels aside Tthe condensation graph $\bar{G}$ of a graph $G$ is the graph whose vertices are the strongly connected components of $G \Gamma$ and where there is an edge from $C_{1}$ to $C_{2}$ if and only if there is an edge $v_{1} \Leftrightarrow v_{2}$ in $G \Gamma$ with $v_{1}$ a vertex of $C_{1}$ and $v_{2}$ a vertex of $C_{2}$. We let $\bar{v}$ be the strongly connected component of $v$. Observe that $\bar{G}$ is then a directed acyclic graph $\Gamma$ or intuitively a "tree with shared subtrees".

Say that the value of a vertex under $\rho$ is $\ell$ if the vertex is a level constant $\ell \Gamma$ and $\rho(\mu)$ if it is a level variable $\mu$. We write (abusively) $\rho(v)$ the value of $v$ under $\rho$.

Lemma 16 If $\rho$ satisfies $K$, then for every strongly connected component $C$ of $G(K)$, there is a unique level $\ell$ such that for every vertex $v$ of $C, \rho(v)=\ell$. We write this level $\rho(C)$.

Proof: Notice that for any edge $v_{1} \stackrel{n}{\leftrightarrow} v_{2}$ Twe must have $\rho\left(v_{1}\right) \geq \rho\left(v_{2}\right)+n \Gamma$ with $n \geq 0 \Gamma$ and therefore $\rho\left(v_{1}\right) \geq \rho\left(v_{2}\right)$. Now if there is a path from $v_{1}$ to $v_{2} \Gamma$ then by induction on the length of the path $\Gamma$ $\rho\left(v_{1}\right) \geq \rho\left(v_{2}\right)$. In a strongly connected component $C \Gamma$ for any two distinct vertices $v_{1}$ and $v_{2}$ Tby definition $v_{2}$ is reachable from $v_{1} \Gamma$ so $\rho\left(v_{1}\right) \geq \rho\left(v_{2}\right) \Gamma$ and $v_{1}$ is reachable from $v_{2} \Gamma$ so $\rho\left(v_{2}\right) \geq \rho\left(v_{1}\right)$. It follows that $\rho\left(v_{1}\right)=\rho\left(v_{2}\right)$.

Lemma 17 If $\rho$ satisfies $K$, then in every strongly connected component $C$ of $G(K)$, all the weights labeling edges inside $C$ are 0 .

Proof: Let $v_{1} \stackrel{n}{\leftrightarrow} v_{2}$ be an edge in $C$. By Lemma $16 \Gamma$ $\rho\left(v_{1}\right)=\rho\left(v_{2}\right)$. But since $\rho$ satisfies $K \Gamma \rho\left(v_{1}\right) \geq$ $\rho\left(v_{2}\right)+n$ Thence $n=0$. 
We say that a strongly connected component is consistent if and only if all its edges have weights equal to $0 \Gamma$ and it contains at most one constant level vertex. If it contains one such constant vertex $\ell \Gamma$ then we say that it is fixed Totherwise it is variable.

Lemma 18 There is a polynomial-time algorithm which, given any system $K$ of constraints, decides whether it is satisfiable, and if so, returns the least assignment $\rho$ on $\operatorname{dom} K$ satisfying $K$ with respect to the pointwise ordering on ordinals.

Proof: Build the condensation graph $\bar{G}$ of $G(K) \Gamma$ and label each edge $C_{1} \Leftrightarrow \rightarrow C_{2}$ in $\bar{G}$ by the least upper bound $n$ of all weights $m$ of edges $v_{1} \stackrel{m}{\leftrightarrow} v_{2} \Gamma$ with $v_{1}$ a vertex of $C_{1}$ and $v_{2}$ a vertex of $C_{2}$. This can be done in polynomial time by a slight variant of Tarjan's algorithm for finding strongly connected components [McH90].

Now decorate each vertex of $\bar{G}$ in reverse topological order (i.e. $\Gamma$ bottom-up $\Gamma$ since this graph is acyclic) as follows. At vertex $C \Gamma$ with immediate successors $C_{1} \Gamma \ldots \Gamma C_{k}$ reached respectively through edges of weights $n_{1} \Gamma \ldots \Gamma n_{k}$ (at this step $C_{1} \Gamma \ldots \Gamma$ $C_{k}$ have already been decorated $\Gamma$ say with respective levels $\left.\ell_{1} \Gamma \ldots \Gamma \ell_{k}\right)$ :

1. Check whether $C$ is consistent; if not $\Gamma$ then fail: $K$ is unsatisfiable. Otherwise $\Gamma$ do:

2. If $C$ is fixed $\Gamma$ then let $\ell$ be the unique constant vertex in $C$; if $\ell<\ell_{i}+n_{i}$ for some $i \Gamma 1 \leq i \leq k \Gamma$ then fail: $K$ is unsatisfiable. Otherwise $\overline{\text { decorate }}$ $C$ with $\ell$.

3. If $C$ is variableTthen decorate $C$ with $\max \left(\ell_{1}+\right.$ $\left.n_{1}, \ldots, \ell_{k}+n_{k}\right)$.

This algorithm clearly runs in polynomial time in the size of the input $K$. If it succeeds $\Gamma$ let $\rho_{0}$ be the assignment that maps each level variable $\mu$ to the decoration of $\bar{\mu}$. We now show that the algorithm answers the question.

$(\Longrightarrow)$ Assume that $K$ is satisfiable. We claim that the algorithm does not fail $\Gamma$ and that every assignment $\rho$ satisfying $K$ is (pointwise) greater than or equal to $\rho_{0}$. We prove the claim by well-founded induction on the directed acyclic graph $\bar{G}$ Tshowing at each step (where $C$ is the currently examined vertex of $\bar{G})$ that $\rho$ is pointwise at least $\rho_{0}$ on all vertices of $G(K)$ reachable from vertices in $C$.

So consider the current vertex $C$ in $\bar{G} T C \stackrel{n_{i}}{\leftrightarrow} C_{i} \Gamma$ $1 \leq i \leq k$ T be the outgoing edges. By Lemma $16 \Gamma C$ contains at most one level constant Tand by Lemma 17 all the edges in $G$ between elements of $C$ have weight
0Tso $C$ is consistent: the algorithm therefore does not fail at step 1.

If $C$ is fixed then necessarily $\rho(C)=\ell$ where $\ell$ is the unique constant in $C$. Consider a fixed arbitrary $C_{i} \Gamma 1 \leq i \leq k$. For every edge $v_{1} \stackrel{n}{\leftrightarrow} v_{2}$ in $G$ going from a vertex $v_{1}$ in $C$ to a vertex $v_{2}$ in $C_{i} \Gamma$ we have $\rho\left(v_{1}\right) \geq \rho\left(v_{2}\right)+n$. Because $n_{i}$ is the least upper bound of all such $n \Gamma \rho(C) \geq \rho\left(C_{i}\right)+n_{i}$; this is in turn at least $\rho_{0}\left(C_{i}\right)+n_{i}$ by induction hypothesis $\Gamma$ that is $\Gamma$ we have $\ell>\ell_{i}+n_{i}$ for every $i \Gamma 1 \leq i \leq k \Gamma$ where $\ell_{i}=\rho_{0}\left(C_{i}\right)$. Therefore $\Gamma$ the algorithm does not fail at step 2 either. Moreover for every vertex $v$ in $C \Gamma$ $\rho(v)=\ell=\rho_{0}(v) \Gamma$ and for every vertex $v$ reachable from some $C_{i} \Gamma 1 \leq i \leq k \Gamma \rho(v) \geq \rho_{0}(v)$ by induction hypothesis. So $\rho$ is pointwise at least $\rho_{0}$ on vertices reachable from $C$.

If $C$ is variable $\Gamma$ then the algorithm cannot fail. Moreover Tby a similar argument $\rho(C) \geq \rho\left(C_{i}\right)+n_{i}$ for every $i \Gamma 1 \leq i \leq k$. By induction hypothesis $\rho\left(C_{i}\right) \geq$ $\rho_{0}\left(C_{i}\right)=\ell_{i} \Gamma$ so $\rho(C) \geq \max \left(\ell_{1}+n_{1}, \ldots, \ell_{k}+n_{k}\right)=$ $\rho_{0}(C)$. The claim is then proved.

$(\Longleftarrow)$ Assume that the algorithm does not fail. We claim that $\rho_{0}$ satisfies $K$. Again we prove it by wellfounded induction on $\bar{G}$ Tshowing at each step (where the current vertex in $\bar{G}$ is $C$ ) that $\rho_{0}$ satisfies all the constraints described by edges of $G(K)$ that are reachable from vertices in $C$.

So let $C$ be the current vertex in $\bar{G} \Gamma C \stackrel{n_{i}}{\leftrightarrow} C_{i} \Gamma$ $1<i<k$ Tbe the outgoing edges. By induction hypothesis $\Gamma \rho_{0}$ satisfies all the constraints in $K$ represented by edges in $C_{1} \Gamma \ldots \Gamma C_{k}$ or below. We show that it also satisfies all constraints represented by edges of $G(K)$ inside $C \Gamma$ and by edges of $G(K)$ going from vertices inside $C$ to vertices inside $C_{i}$.

Since the algorithm does not fail at step 1. $C$ is consistent. Let then be $v_{1} \Leftrightarrow{ }^{0} v_{2}$ any edge from vertex $v_{1}$ in $C$ to vertex $v_{2}$ in $C$. This corresponds to the constraint $\rho_{0}\left(v_{1}\right) \geq \rho_{0}\left(v_{2}\right) \Gamma$ which is trivially satisfied since $\rho_{0}\left(v_{1}\right)=\rho_{0}\left(v_{2}\right)=\rho_{0}(C)$.

Moreover $\Gamma \rho_{0}$ is such that $\rho_{0}(C) \geq \max \left(\rho_{0}\left(C_{1}\right)+\right.$ $\left.n_{1}, \ldots, \rho_{0}\left(C_{k}\right)+n_{k}\right) \Gamma$ whether $C$ is fixed or variable. So let $v_{1} \stackrel{n}{\leftrightarrow} v_{2}$ be any edge in $G(K)$ from a vertex $v_{1}$ in $C$ to a vertex $v_{2}$ in some $C_{i} \Gamma 1 \leq$ $i \leq k$. Then $\rho_{0}\left(v_{1}\right)=\rho_{0}(C)$ (by definition) $\geq$ $\max \left(\rho_{0}\left(C_{1}\right)+n_{1}, \ldots, \rho_{0}\left(C_{k}\right)+n_{k}\right)$ (by the remark above) $\geq \rho_{0}\left(C_{i}\right)+n_{i}=\rho_{0}\left(v_{2}\right)+n_{i}$ (by definition of $\left.\rho_{0}\right) \geq \rho_{0}\left(v_{2}\right)+n$ (by definition of $\left.n_{i}\right)$. So the constraint represented by this edge is again satisfied.

It would be interesting to transform this algorithm into an incremental algorithmГi.e. an algorithm where new constraints are progressively added and satisfiability is checked at each step. We leave this as an improvement to be done. 


\subsection{Level Inference}

We can then translate conditions for being well-typed $\Gamma$ for two types to be less or equalTand so on Tas systems of constraints. We let $\mu=\nu$ denote the set of two constraints $\mu \geq \nu \Gamma \nu \geq \mu$.

Lemma 19 Given any ramified pre-types $\tau, \tau^{\prime}$, and level variable $\mu$, we can build in polynomial time systems of constraints $K(l(\tau) \leq \mu), K(\tau$ ramified $)$, $K\left(\tau \sqsubseteq \tau^{\prime}\right)$ and $K\left(\tau=\tau^{\prime}\right)$ such that the assignments that satisfy them are precisely those under which $l(\tau) \leq \mu, \tau$ is a ramified type, $\tau \sqsubset \tau^{\prime}$ and $\tau=\tau^{\prime}$ respectively.

Proof: In the following Tlet $b$ stand for base typesT $\tau$ for any ramified type (possibly primed or indexed). Let $T$ (true) denote the empty set $\emptyset$ of constraints $\Gamma$ and $\perp$ (false) denote any unsatisfiable set of constraints $\Gamma$ like $\{0 \geq \mu, \mu \geq 1\}$ for some variable $\mu$.

$$
\begin{array}{ll}
K(l(b) \leq \mu) & = \\
K\left(l\left(\tau_{1} \stackrel{\nu}{\rightarrow} \tau_{2}\right) \leq \mu\right) & =\{\mu \geq \nu+1\} \cup K \\
& =\top \\
K(b \text { ramified }) & =K\left(\tau_{1} \text { ramified }\right) \\
K\left(\tau_{1} \stackrel{\nu}{\rightarrow} \tau_{2} \text { ramified }\right) & \\
& \cup K\left(\tau_{2} \text { ramified }\right) \\
& \cup K\left(l\left(\tau_{1}\right) \leq \nu\right)
\end{array}
$$$$
K\left(l\left(\tau_{1} \stackrel{\nu}{\rightarrow} \tau_{2}\right) \leq \mu\right) \quad=\{\mu \geq \nu+1\} \cup K\left(l\left(\tau_{2}\right) \leq \mu\right)
$$

$$
\begin{array}{ll}
K(b \sqsubseteq b) & =\top \\
K\left(\tau_{1} \stackrel{\mu}{\rightarrow} \tau_{2} \sqsubseteq \tau_{1}^{\prime} \stackrel{\mu^{\prime}}{\rightarrow} \tau_{2}^{\prime}\right) & =K\left(\tau_{1}^{\prime} \sqsubseteq \tau_{1}\right) \cup K\left(\tau_{2} \sqsubseteq \tau_{2}^{\prime}\right) \\
K\left(\tau \sqsubseteq \tau^{\prime}\right) & \cup\left\{\mu \geq \mu^{\prime}\right\} \cup K\left(l\left(\tau_{1}^{\prime}\right) \leq \mu^{\prime}\right) \\
K & =\perp \text { otherwise }
\end{array}
$$

In the latter clause $\Gamma$ it will be interesting in practice to just fail Taborting the whole computationTand returning $\perp$.

$$
\begin{array}{ll}
K(b=b) & =\top \\
K\left(\tau_{1} \stackrel{\mu}{\rightarrow} \tau_{2}=\tau_{1}^{\prime} \stackrel{\mu^{\prime}}{\rightarrow} \tau_{2}^{\prime}\right) & =K\left(\tau_{1}=\tau_{1}^{\prime}\right) \cup K\left(\tau_{2}=\tau_{2}^{\prime}\right) \\
& \cup\left\{\mu=\mu^{\prime}\right\} \\
K\left(\tau \sqsubseteq \tau^{\prime}\right) & =\perp \text { otherwise }
\end{array}
$$

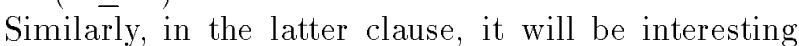
in practice to just fail.

Let $\mathrm{FLV}(s)$ be the set of free level variables in the pre-term $s \Gamma$ defined as $\operatorname{FLV}\left(\lambda x_{\epsilon} \cdot u\right)=\operatorname{FLV}(u) \Gamma$ $\operatorname{FLV}(u v)=\operatorname{FLV}(u) \cup \operatorname{FLV}(v) \Gamma \operatorname{FLV}\left(x_{\tau}^{\mu}\right)=\{\mu\} \cup$ $\operatorname{FLV}(\tau)$ Tand where $\operatorname{FLV}(\tau)$ is defined as $\operatorname{FLV}(b)=\emptyset$ if $b$ is a base type $\Gamma$ and $\operatorname{FLV}\left(\tau_{1} \stackrel{\mu}{\rightarrow} \tau_{2}\right)=\operatorname{FLV}\left(\tau_{1}\right) \cup$ $\operatorname{FLV}\left(\tau_{2}\right) \cup\{\mu\}$.

We wish to find a system of level constraints that would express exactly when a given pre-term $s$ has a ramified type. This system of level constraints should therefore have FLV $(s)$ as domain. But a predicate denoting typability of $s$ will be of the form $\exists \mu_{1}, \ldots, \mu_{k} \cdot K \Gamma$ where $K$ is a system of constraints expressing all the ordering constraints between level variables that occur in a derivation $\Gamma$ and $\mu_{1} \Gamma \ldots \Gamma \mu_{k}$ are unknowns that must be introduced to represent unknown levels in $\left(V a r^{\prime}\right)$ and $(A b s)$. We might show that we can represent such predicates as systems of constraints (to represent $\exists \mu \cdot K$ T the rough idea is to add edges $v \stackrel{m+n}{\leftrightarrow} v^{\prime}$ to $G(K)$ whenever we have edges $v \stackrel{m}{\leftrightarrow} \mu$ and $\mu \stackrel{n}{\leftrightarrow} v^{\prime}$ Tthen to eliminate all edges incident on $\mu$; the real procedure is a bit more complicated).

But it will be easier to leave the existentially quantified variables explicit Tand instead to consider a refined notion of satisfaction. For any set $S$ of level variables $\Gamma$ we say that a level assignment $\rho$ satisfies a system of constraints $K$ up to $S$ if and only if there is a level assignment $\rho^{\prime}$ satisfying $K$ such that $\rho(\mu)=\rho^{\prime}(\mu)$ for every $\mu \in S$. (That is $\Gamma$ we quantify on all variables in $\operatorname{dom} K \backslash S$.)

\section{Theorem 20 (Level Reconstruction)}

Typability of ramified pre-terms is decidable in polynomial time.

More precisely, there is a polynomial-time algorithm which, given a ramified pre-term s, either fails if $s$ is not typable, or returns a ramified pre-type $\tau_{s}$, a level variable $\mu_{s}$ and a system of constraints $K\left(s: \tau_{s} / \mu_{s}\right)$ such that $s$ is well-typed and has decorated type $\tau_{s} / \mu_{s}$ under assignment $\rho$ if and only if $\rho$ satisfies $K\left(s: \tau_{s} / \mu_{s}\right)$ up to $\operatorname{FLV}(s)$.

Proof: This is a more or less direct translation of the rules used in normal derivations (but we might as well do this directly on the rules of Figure 1Talthough this would be less efficient). We first define a suitable notion of occurrence $p$ in a pre-term or pre-type. Occurrences will be words on the alphabet $\{0,1\}$. We define the set of occurrences of $u$ and the subterm $\left.u\right|_{p}$ of $u$ at occurrence $p$ inductively as follows. The empty word $\varepsilon$ is an occurrence in every term $u$ T and $\left.u\right|_{\varepsilon}=u$; if $p$ is an occurrence in $u \Gamma$ and $\left.u\right|_{p}$ is an abstraction $\lambda x_{\epsilon} \cdot v$ T then $p 0$ is an occurrence in $u$ and $\left.u\right|_{p 0}=v$; if $\left.u\right|_{p}$ is an application $v w \Gamma$ then $p 0$ and $p 1$ are occurrences in $u$ and $\left.u\right|_{p 0}=\left.v \Gamma u\right|_{p 1}=w$. Similarly $\epsilon_{p}=\epsilon_{1} \rightarrow \epsilon_{2} \Gamma$ then $p 0$ and $p 1$ are occurrences in $\epsilon$ and $\left.\epsilon\right|_{p 0}=\left.\epsilon_{1} \Gamma \epsilon\right|_{p 1}=\epsilon_{2}$.

First $\Gamma$ for every subterm occurrence $p$ in $s \Gamma$ let $\mu_{p}$ be a level variable (this will denote the level of $\left.s\right|_{p}$ ). For every occurrence of an abstraction $\lambda x_{\epsilon} \cdot t$ in $s$ Tlet $\nu_{x}$ and $\nu_{x}^{p}$ be level variables $\Gamma$ for every occurrence $p$ of a functional subtype $\epsilon_{1} \rightarrow \epsilon_{2}$ in $\epsilon$; we let $\theta_{x}$ be the ramified pre-type obtained by recursively decorating each functional subtype at occurrence $p$ in $\epsilon$ as above by $\nu_{x}^{p}$ (intuitively $\Gamma \theta_{x} / \nu_{x}$ denotes the decorated type of the variable $y$ to guess in rule $(A b s)$ ). FinallyTfor 
every occurrence $p$ of a constant or a variable (free or bound) $x_{\epsilon}$ (resp. $x_{\tau}^{\ell}$ ) for every occurrence $q$ of a functional subtype $\epsilon_{1} \rightarrow \epsilon_{2}$ in $\epsilon$ (resp. $\left.E(\tau)\right)$ Tlet $\xi_{p}^{q}$ be a level variable; similarlyГwe define $\theta_{p}$ as the ramified pre-type obtained by recursively decorating each functional subtype at occurrence $q$ in $\epsilon$ as above by $\xi_{p}^{q}$ (intuitively $\theta_{p} / \mu_{p}$ will be the decorated type that we assign to $x$ by rule $\left.\left(\operatorname{Var}^{\prime}\right)\right)$. We assume that all these level variables are pairwise distinct and distinct from the level variables in $\operatorname{FLV}(s)$.

We arrange the set of occurrences in $s$ as a finite tree by sharing occurrence prefixes (this tree is the skeleton of $s$ ). The algorithm is then defined by structural recursion on this tree Treturning a pre-type $\tau_{p}(\sigma)$ and a set of constraints $K_{p}(\sigma)$ at each occurrence $p$ Twhere $\sigma$ is a substitution mapping all variables $x_{\epsilon}$ bound in $s$ but possibly free in $\left.s\right|_{p}$ to variables of the form $y_{\tau}^{\ell}$. (The purpose of $\sigma$ is to effect all substitutions needed in rule $(A b s)$ in a lazy way.)

\section{Algorithm 21 For each occurrence $p$ in $s$ :}

(Variable case) If $p$ is an occurrence of a constant or a variable, equal to or mapped by $\sigma$ to $x_{\tau}^{\mu}$, then let $\tau_{p}(\sigma)$ be $\theta_{p}$, and $K_{p}(\sigma)$ be $K\left(\tau \sqsubseteq \theta_{p}\right) \cup\left\{\mu_{p} \geq\right.$ $\mu\} \cup K(l(\tau) \leq \mu)$ (rule $\left.\left(\operatorname{Var}^{\prime}\right)\right)$.

(Application case) If $p$ is an occurrence of an application $u v$, then check that $\tau_{p 0}(\sigma)$ (intuitively, the type of $u$ ) is of the form $\tau_{1} \stackrel{\xi}{\rightarrow} \tau_{2}$, with $E\left(\tau_{1}\right)=$ $E\left(\tau_{p 1}(\sigma)\right)$; otherwise, fail ( $s$ is not typable). Let then $\tau_{p}(\sigma)$ be $\tau_{2}$, and $K_{p}(\sigma)$ be $K_{p 0}(\sigma) \cup K_{p 1}(\sigma) \cup K\left(\tau_{1}=\right.$ $\left.\tau_{p 1}\right) \cup\left\{\xi=\mu_{p 1}, \mu_{p}=\mu_{p 0}\right\}($ rule $(A p p))$.

(Abstraction case) If $p$ is an occurrence of an abstraction $\lambda x_{\epsilon} \cdot v$, then let $y_{\theta_{x}}^{\nu_{x}}$ be a variable not free in $v, \tau_{p}(\sigma)=\theta_{x} \stackrel{\nu_{x}}{\rightarrow} \tau_{p 0}\left(\sigma\left[y_{\theta_{x}}^{\nu_{x}} / x\right]\right)$, and $K_{p}(\sigma)$ be $K_{p 0}\left(\sigma\left[y_{\theta_{x}}^{\nu_{x}} / x\right]\right) \cup K\left(\theta_{x}\right.$ ramified $) \cup K\left(l\left(\theta_{x}\right) \leq \nu_{x}\right\} \cup$ $\left\{\mu_{p} \geq \nu_{x}+1, \mu_{p} \geq \mu_{p 0}\right\}$ (rule $(A b s) ;$ notice that we don't encode $\mu_{p}=\max \left(\nu_{x}+1, \mu_{p 0}\right)$, but $\mu_{p} \geq$ $\max \left(\nu_{x}+1, \mu_{p 0}\right)$, but this is all right, as we shall see).

Finally, we let $\tau_{s}$ be $\tau_{\varepsilon}([]), \mu_{s}$ be $\mu_{\varepsilon}$, and $K(s$ : $\left.\tau_{s} / \mu_{s}\right)$ be $K_{\varepsilon}([])$.

Proof of Correctness: We now prove that the algorithm is correct. We claim that for each occurrence $p \Gamma(1)$ if $\tau_{p}(\sigma)$ is undefined $\Gamma$ then $\left.u\right|_{p} \sigma$ is not typable $\Gamma$ and (2) if $\tau_{p}(\sigma)$ is defined $\Gamma$ then the assignments $\rho$ under which $\left.u\right|_{p} \sigma$ is typable are exactly those which satisfy $K_{p}(\sigma)$ up to $\operatorname{FLV}\left(\left.u\right|_{p} \sigma\right) \Gamma$ and that its type is then $\tau_{p} / \mu_{p}$. We prove the claim by structural induction on the tree of occurrences.

If $p$ is an occurrence of a variableTthen $\left.u\right|_{p} \sigma$ is some $x_{\tau}^{\mu}$. (1) is trivial since $\tau_{p}(\sigma)$ is always defined. As for (2) $\Gamma$ if $x$ is typable of type $\tau^{\prime} / \mu_{p}$ under $\rho \Gamma$ then we must have $\mu \geq l(\tau) \Gamma$ and the type has been obtained by rule $\left(\operatorname{Var}^{\prime}\right) \Gamma$ so $\tau \sqsubseteq \tau^{\prime}$ and $\mu \leq \mu_{p}$. From $\tau \sqsubseteq \tau^{\prime}$ it follows that $E(\tau)=E\left(\tau^{\prime}\right)$ Thence that the general form of $\tau^{\prime}$ is described by $\theta_{p}$. So $\tau_{p}(\sigma)$ must indeed be $\theta_{p}$ with $K_{p}(\sigma)$ satisfied. Conversely $\Gamma$ if $K_{p}(\sigma)$ is satisfied by $\rho \Gamma$ then under $\rho$ we have $\tau \sqsubseteq \theta_{p} \Gamma$ hence by Lemma $2 \tau$ is a ramified type; moreover $l(\tau) \leq \mu \Gamma$ $\tau \sqsubseteq \theta_{p}$ and $\mu \leq \mu_{p}$ under $\rho \Gamma$ so by rule $\left.\left(V a r^{\prime}\right) u\right|_{p} \sigma$ has indeed type $\theta_{p} / \mu_{p}$.

Assume that $p$ is an occurrence of an application $u v$. If the algorithm fails then either it failed in $u$ or in $v$ Tin which case $(u v) \sigma$ is not typable by induction hypothesisTclaim (1); or it fails because $\tau_{p 0}(\sigma)$ is not an functional type or $E\left(\tau_{1}\right) \neq E\left(\tau_{p 1}(\sigma)\right)$ : in each case $\Gamma$ rule $(A p p)$ cannot be applied $\Gamma$ so $(u v) \sigma$ is not typable (this is by Theorem 6). This proves (1). As for (2) $\Gamma$ if $(u v) \sigma$ is typable $\Gamma$ then $u$ and $v$ are $\Gamma$ too $\Gamma$ the type $\tau_{1}$ of $u$ 's argument must equal the type $\tau_{p 1}$ of $v \Gamma$ the level $\mu_{1}$ of $u$ 's argument must equal the level $\mu_{p 1}$ of $v$ Tand the level $\mu_{p}$ of $(u v) \sigma$ must equal that of $u \sigma \Gamma$ namely $\mu_{p 0}$ : this yields all the constraints in $K_{p}(\sigma)$. Moreover $\Gamma$ the type returned must be the type $\tau_{2}$ of the results of $u \sigma$. ConverselyCit is clear that if $K_{p}(\sigma)$ is satisfied by $\rho$ Then by induction hypothesis we have type derivations of $u \sigma: \tau_{1} \stackrel{\mu_{1}}{\rightarrow} \tau_{2} / \mu_{p 0}$ and $v \sigma: \tau_{1} / \mu_{1} \Gamma$ so that we can apply rule $(A p p)$ to get $(u v) \sigma: \tau_{2} / \mu_{p}$.

Finally assume that $p$ is an occurrence of an abstraction $\lambda x_{\epsilon} \cdot v$. Claim (1) is clear from the induction hypothesis. As for (2) $\Gamma$ if $\left(\lambda x_{\epsilon} \cdot v\right) \sigma$ is typable under $\rho$ Then by Theorem 6 the last rule in the derivation can be assumed to be $(A b s) \Gamma$ so there is a variable $y_{\theta_{x}}^{\nu_{x}}$ not free in $v$ such that $E\left(\theta_{x}\right)=\epsilon$ and $v \sigma\left[y_{\theta_{x}}^{\nu_{x}} / x\right]$ is typable. By induction hypothesis $\Gamma$ $K_{p 0}\left(\sigma\left[y_{\theta_{x}}^{\nu_{x}} / x\right]\right)$ must therefore be satisfied by $\rho$. And $\theta_{x}$ must be a ramified type $\Gamma$ with $\nu_{x} \geq l\left(\theta_{x}\right)$ so $K\left(\theta_{x}\right.$ ramified $) \cup K\left(l\left(\theta_{x}\right) \leq \nu_{x}\right)$ must also be satisfied by $\rho$. Moreover $\Gamma$ we must have $\mu_{p}=\max \left(\nu_{x}+1, \mu_{p 0}\right) \Gamma$ so in particular $\mu_{p} \geq \nu_{x}+1, \mu_{p} \geq \mu_{p 0}$; hence $\Gamma K_{p}(\sigma)$ is satisfied by $\rho$. And the type of $\left(\lambda x_{\epsilon} \cdot v\right) \sigma$ must then be $\theta_{x} \stackrel{\nu_{x}}{\rightarrow} \tau_{p 0}\left(\sigma\left[y_{\theta_{x}}^{\nu_{x}} / x\right]\right)$ Ti.e. $\tau_{p}(\sigma)$. Conversely $\Gamma$ if $K_{p}(\sigma)$ is satisfied $\Gamma$ then it is clear by induction hypothesis that under any $\rho$ satisfying it $\Gamma$ we can infer $\left(\lambda x_{\epsilon} \cdot v\right) \sigma: \theta_{x} \stackrel{\nu_{x}}{\rightarrow} \tau_{p 0}\left(\sigma\left[y_{\theta_{x}}^{\nu_{x}} / x\right]\right) / \max \left(\nu_{x}+1, \mu_{p 0}\right)$ Гi.e. $\left(\lambda x_{\epsilon} \cdot v\right) \sigma: \tau_{p}(\sigma) / \max \left(\nu_{x}+1, \mu_{p 0}\right)$ Twhere $\theta_{x}$ is ramified and $\nu_{x} \geq l\left(\theta_{x}\right)$. To infer $\left(\lambda x_{\epsilon} \cdot v\right) \sigma: \tau_{p}(\sigma) / \mu_{p} \Gamma$ since $\mu_{p} \geq \max \left(\nu_{x}+1, \mu_{p 0}\right)$ Twe just apply rule $(\mathrm{Cml})$ (and apply Lemma 5 if we insist on getting normal derivations).

Running Time: FinallyC Algorithm 21 runs in polynomial time. We assume that $\sigma$ is represented by a balanced tree or any data structure where adding an element and retrieving an element is fast - typically in time logarithmic in the cardinality of $\sigma$ Ti.e. at most $O(\log |s|)$. Furthermore $\Gamma$ we assume that unions of 
sets of constraints are done by adding one edge at a time to a global graph; for exampleTthe behavior on applications is just adding the edges resulting from $\tau_{1}=\tau_{p 1} \Gamma \mu_{1}=\mu_{p 1}$ and $\mu_{p}=\mu_{p 0}$ to the global graph.

We then claim that computing $\tau_{\varepsilon}([])$ and $K_{\varepsilon}([])$ takes $O\left(|s|^{2} \log |s|\right.$ ) time $\Gamma$ for any $\alpha>1$ (more precisely $\mathrm{T}$ time bounded by $\left.k|s|^{2} \log (|s|+1)\right)$. To show this $\Gamma$ we prove by structural induction on the occurrence tree that computing $\tau_{p}(\sigma)$ and $K_{p}(\sigma)$ takes $k|u|^{2} \log (|s|+1)$ time $\Gamma$ where $u=\left.s\right|_{p} \Gamma$ and that $\left|\tau_{p}(\sigma)\right| \leq O(|u|)$.

Each variable step $\Gamma$ for a variable equal to or mapped by $\sigma$ to $x_{\tau}^{\mu}$ adds at most $O(|\tau|)$ new edges to the global graph $\Gamma$ and therefore takes at most $O\left(\left|x_{\tau}^{\mu}\right| \times \log |s|\right) \leq k\left|x_{\tau}^{\mu}\right|^{2} \log (|s|+1)$. Moreover $\Gamma$ the returned type has the same size as $\tau$ Ti.e. less than $O\left(\left|x_{\tau}^{\mu}\right|\right)$.

Each application step (where $\left.s\right|_{p}=u v$ ) needs time $O\left(\left|\tau_{p 1}(\sigma)\right|\right)$ to test for failure ( $u$ having non-functional type or $\left.E\left(\tau_{1}\right) \neq E\left(\tau_{p 1}(\sigma)\right)\right) \Gamma$ and adds $O\left(\left|\tau_{p 1}(\sigma)\right|\right)$ new edges to the global graph. This needs at most $O\left(\left|\tau_{p 1}(\sigma)\right|\right)=O(|v|)$ time by induction hypothesis. Now typing $u$ took time at most $k|u|^{2} \log (|s|+1) \Gamma$ typing $v$ took $k|v|^{2} \log (|s|+1) \Gamma$ so all in all we need time $k|u|^{2} \log (|s|+1)+k|v|^{2} \log (|s|+1)+k^{\prime}|v|+$ $o(|v|)$ to check $u v$. But since $|u v|=|u|+|v|+1 \Gamma$ $k|u v|^{2} \log (|s|+1) \geq k|u|^{2} \log (|s|+1)+k|v|^{2} \log (|s|+$ $1)+2 k|u||v| \log (|s|+1)$. So $\Gamma$ provided that $k$ is high enough that $2 k|u| \log (|s|+1) \geq k^{\prime}$ (i.e. for example $\left.k \geq k^{\prime} /(2 \log 2)\right) \Gamma$ the time taken is bounded above by $k|u v|^{2} \log (|s|+1)$. Moreover $\Gamma$ we have $\left|\tau_{p}(\sigma)\right| \leq$ $O(|u|) \leq O(|u v|)$

Each abstraction step (where $\left.s\right|_{p}=\lambda x_{\epsilon} \cdot u$ ) needs $O(|\epsilon|)$ time to build $\theta_{x} \Gamma O(\log |s|)$ time to add the binding $y_{\theta_{x}}^{\nu_{x}} / x$ to $\sigma \Gamma k|u|^{2} \log (|s|+1)$ time to type $u \Gamma$ and adds $O(|\epsilon|)$ edges to the global graph. Therefore it takes at most $k|u|^{2} \log (|s|+1)+O(\log |s|)+O(|\epsilon|)$ time Twhich is (much) less that $k\left|\lambda x_{\epsilon} \cdot u\right|^{2} \log (|s|+1) \Gamma$ since $\left|\lambda x_{\epsilon} \cdot u\right|=|\epsilon|+|u|+1$. And the returned type $\tau_{p}(\sigma)$ is $\theta_{x} \stackrel{\nu_{x}}{\rightarrow} \tau_{p 0}\left(\sigma\left[y_{\theta_{x}}^{\nu_{x}} / x\right]\right)$ Twhere by induction hypothesis $\left|\tau_{p 0}\left(\sigma\left[y_{\theta_{x}}^{\nu_{x}} / x\right]\right)\right| \leq O(|u|) \Gamma$ and by construction $\left|\theta_{x}\right| \leq|\epsilon|$ Tso the returned type has size $O\left(\left|\lambda x_{\epsilon} \cdot u\right|\right)$.

The claim is proved. The total time is then $O\left(|s|^{2} \log |s|\right)$ : this is polynomial in $|s|$.

Corollary 22 Testing whether a given term is typable is decidable in polynomial time.

Proof: Apply Algorithm $21 \Gamma$ and then apply the algorithm of Lemma 18 on the graph $K\left(s: \tau_{s} / \mu_{s}\right)$ Tof size $O\left(|s|^{2}\right)$.

\subsection{Example}

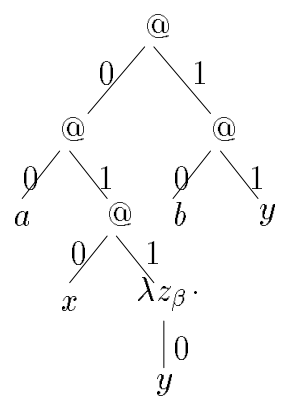

Figure 3: Term example

For example $\Gamma$ consider the term $a\left(x\left(\lambda z_{\beta} \cdot y\right)\right)(b y) \Gamma$ where $\beta$ is a base type $\Gamma a=a_{\beta \rightarrow \beta \rightarrow}^{\ell_{3}} \beta$ and $b=b^{\ell_{5}}{ }_{\beta \rightarrow}^{\ell_{4}}$ are constants $\Gamma$ and $x=x_{(\beta \rightarrow \beta){ }_{3}^{\ell_{8}} \beta}^{\beta \rightarrow \beta \rightarrow \beta}$ and $y=y_{\beta}^{\ell_{9}}$ are variables. The free level variables are $\ell_{1} \Gamma \ldots \Gamma$ $\ell_{9}$. This term is shown as a tree in Figure 3 . At marks (@) denote application nodes $\Gamma$ and 0's and 1's denote letters forming paths. We create new variables $\mu_{\varepsilon} \Gamma \mu_{0} \Gamma \mu_{00} \Gamma \mu_{01} \Gamma \mu_{010} \Gamma \mu_{011} \Gamma \mu_{0110} \Gamma \mu_{1} \Gamma \mu_{10}$ and $\mu_{11}$ (levels of subterms); $\nu_{z}$ (and $\theta_{z}=\beta$ ); $\xi_{00}^{\varepsilon} \Gamma$ $\xi_{00}^{1} \Gamma$ (for $\left.a \Gamma\right) \xi_{010}^{\varepsilon} \Gamma \xi_{010}^{0} \Gamma$ (for $\left.x \Gamma\right) \xi_{10}^{\varepsilon}$ (for $b$ ). We have $\theta_{00}=\beta \stackrel{\xi_{00}^{\varepsilon}}{\rightarrow} \beta \stackrel{\xi_{00}^{1}}{\rightarrow} \beta \Gamma \theta_{010}=\left(\beta \stackrel{\xi_{010}^{0}}{\longrightarrow} \beta\right) \stackrel{\xi_{010}^{\varepsilon}}{\rightarrow} \beta \Gamma \theta_{10}=\beta \stackrel{\xi_{10}^{\varepsilon}}{\longrightarrow} \beta \Gamma$ while $\theta_{0110}=\theta_{11}=\beta$.

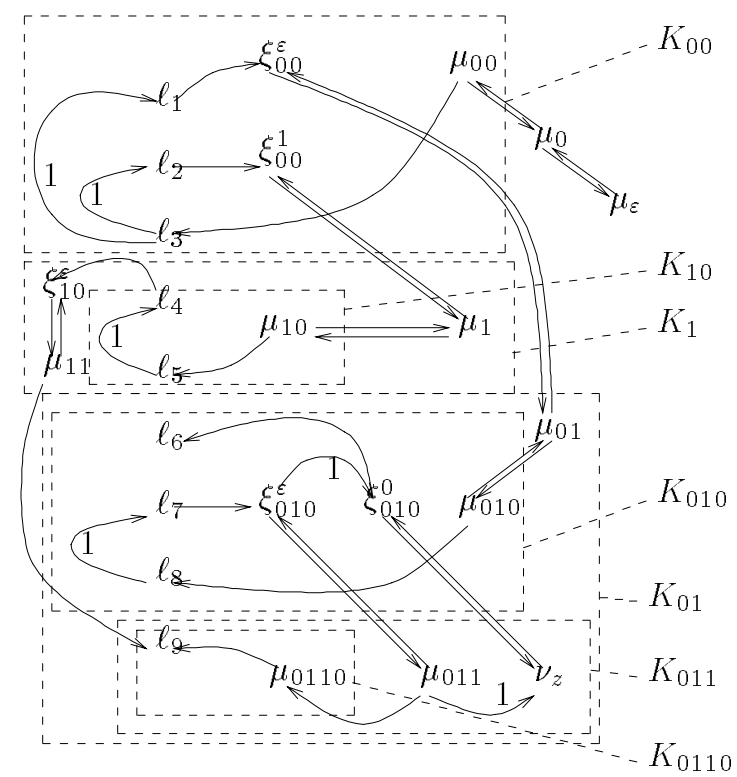

Figure 4: Corresponding graph 
Consider first the occurrence 00 of $a$. Since there is no $\lambda$-header above this occurrence $\Gamma \sigma$ will be the empty substitution. Since $a$ is a constant $\Gamma$ we are in the first case of the Algorithm 21. Then $\tau_{00}(\sigma)=$ $\theta_{00}=\beta \stackrel{\xi_{00}}{\rightarrow} \beta \stackrel{\xi_{00}^{1}}{\rightarrow} \beta$. The constant $a$ is of the form $a_{\tau}^{\mu} \Gamma$ where $\tau=\beta \stackrel{\ell_{1}}{\rightarrow} \beta \stackrel{\ell_{2}}{\rightarrow} \beta$ and $\mu=\ell_{3}$. So $K_{00}(\sigma)=\left\{\ell_{1} \geq\right.$ $\left.\xi_{00}^{\varepsilon}, \ell_{2} \geq \xi_{00}^{1}\right\} \cup\left\{\mu_{00} \geq \ell_{3}\right\} \cup\left\{\ell_{3} \geq \ell_{1}+1, \ell_{3} \geq \ell_{2}+1\right\}$.

Then Tlook at the occurrence 010 of $x$. Again $\sigma$ is the empty substitution $\Gamma$ and we are in the first case of Algorithm 21. $\tau_{010}(\sigma)=\theta_{010}=\left(\beta \stackrel{\xi_{010}^{0}}{\rightarrow} \beta\right) \stackrel{\xi_{010}^{\varepsilon}}{\rightarrow} \beta \Gamma$ $\tau=\left(\beta \stackrel{\ell_{G}}{\rightarrow} \beta\right) \stackrel{\ell_{3}}{\rightarrow} \beta \Gamma \mu=\ell_{8} ;$ so $K\left(\tau \sqsubseteq \theta_{010}\right)=\left\{\xi_{010}^{0} \geq\right.$ $\left.\ell_{6}, \ell_{7} \geq \xi_{010}^{\varepsilon}, \xi_{010}^{\varepsilon} \geq \xi_{010}^{0}+1\right\}$ Tand $\bar{K}_{010}(\sigma)=\left\{\xi_{010}^{0} \geq\right.$ $\left.\ell_{6}, \ell_{7} \geq \xi_{010}^{\varepsilon}, \xi_{010}^{\varepsilon} \geq \xi_{010}^{0}+1, \mu_{010} \geq \ell_{8}, \ell_{8} \geq \ell_{7}+1\right\}$.

The only abstraction in this term is at occurrence 011 . We create a new variable $y_{\theta_{z}}^{\nu_{z}}$ Twhere $\theta_{z}=\beta$ Tand map it to $z$ in $\sigma$. Then $K_{011}(\sigma)=K_{0110}\left(\sigma\left[y_{\theta_{z}}^{\nu_{z}} / z\right]\right) \cup$ $\emptyset \cup \emptyset \cup\left\{\mu_{011} \geq \nu_{z}+1, \mu_{011} \geq \mu_{0110}\right\}$. And the $K_{0110}$ set is just $\left\{\mu_{0110} \geq \ell_{9}\right\}$.

We find an application for example at occurrence 01. The type of the function is $\theta_{010}$ Ti.e. $\left(\beta \stackrel{\xi_{010}^{0}}{\rightarrow} \beta\right) \stackrel{\xi_{010}^{\varepsilon}}{\rightarrow} \beta \Gamma$ and the type of the argument is the type of the abstraction above $\Gamma$ namely $\theta_{z} \stackrel{\nu_{3}}{\rightarrow} \beta=\beta \stackrel{\nu_{3}}{\rightarrow} \beta$. The erasure of the typed expected by the function is $\beta \rightarrow \beta \Gamma$ as is the erasure of the type of the argumentTso Algorithm 21 does not fail (the term is simply-typed). Then $K_{01}(\sigma)=K_{010}(\sigma) \cup K_{011}(\sigma) \cup\left\{\xi_{010}^{0}=\nu_{z}\right\} \cup$ $\left\{\xi_{010}^{\varepsilon}=\mu_{011}, \mu_{01}=\mu_{010}\right\}$ (each of these equalities being really two inequalitiesTi.e. a length 2 cycle in the graph)Tand the returned type is $\beta$.

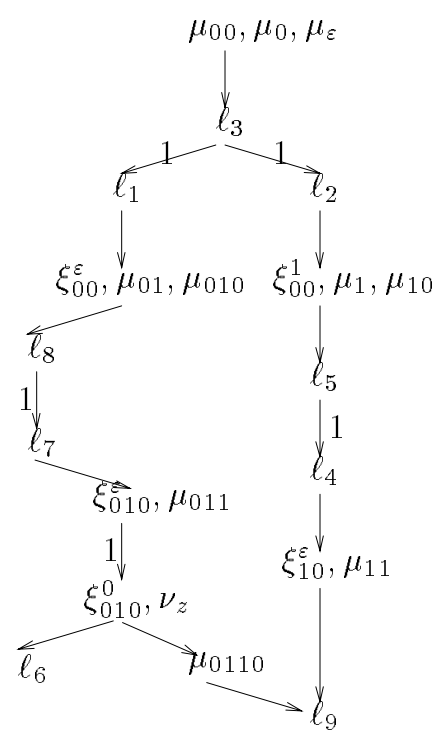

Figure 5: Condensation graph
Continuing this process $\Gamma$ we get as most general type $\beta$ Twith level $\mu_{\varepsilon}$ submitted to the constraints represented in Figure 4. We have omitted all 0 -weights on edgesTso as to make the graph more readable. The condensation graph is shown in Figure 5 ; each $\bar{C}$ is consistent $\Gamma$ so there are level assignments satisfying this set of constraints. The smallest assigns level 3 to the whole expression.

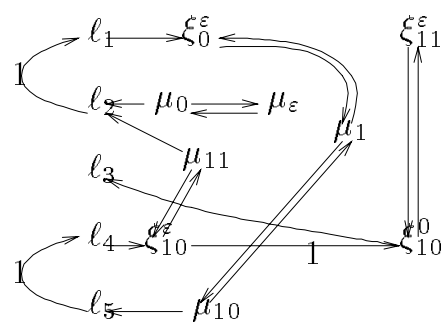

Figure 6: An unsatisfiable graph

Let's take another example $\Gamma$ and consider the term $x(y x) \Gamma$ where $x=x_{\beta \stackrel{\ell_{1}}{\rightarrow} \beta}^{\ell_{2}}$ and $y=y_{\left(\beta \stackrel{\ell_{3}}{\rightarrow} \beta\right) \stackrel{\ell_{4}}{\rightarrow} \beta}^{\ell_{5}}$ By Theorem $8 \Gamma$ this term cannot be typable $\Gamma$ so let's check it. The computed graph is shown in Figure $6 \Gamma$ and has four connected components: $\left\{\ell_{3}\right\} \Gamma\left\{\xi_{10}^{0}, \xi_{11}^{\varepsilon}\right\} \Gamma$ $\left\{\xi_{10}^{\varepsilon}, \mu_{11}, \ell_{2}, \ell_{1}, \xi_{0}^{\epsilon}, \mu_{1}, \mu_{10}, \ell_{5}, \ell_{4}\right\} \Gamma$ and $\left\{\mu_{0}, \mu_{\varepsilon}\right\}$. But the third is inconsistent $\Gamma$ as it contains two edges of non-zero weight (alternatively it contains a cycle of weight 2).

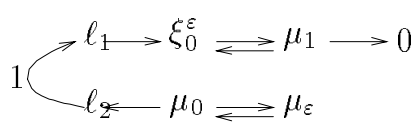

Figure 7: Checking for satisfiable instances

Naturally although $x(y x)$ is untypable $\Gamma$ it has typable instances (modulo $\beta \eta$ ). This comes from Lemma $13 \Gamma$ noticing that $(x(y x) \zeta) \downarrow=\hat{e}_{\beta \rightarrow \beta}$ is clearly typable. If $x$ is a constant and $y$ is a variable $\Gamma$ $x(y x)$ still has typable instances as $(x(y x) \zeta) \downarrow=$ $x \hat{e}_{(\beta \rightarrow \beta) \rightarrow \beta}$ is typable. Indeed $\Gamma$ this term is $x_{\beta \rightarrow \beta}^{\ell_{2}} v_{\beta}^{0} \Gamma$ and its graph is shown in Figure 7 .

\subsection{Improvements}

We can improve Algorithm 21 a great deal. First $\Gamma$ we are not forced to generate all level variables in advance $\Gamma$ and we may create them as we need them.

Then Twe may define $K_{p}$ for variable or constant occurrences $p$ as just $K\left(\tau \sqsubset \theta_{p}\right) \cup\left\{\mu_{p} \geq \mu\right\}$ so as to re- 
duce the number of constraints that we add; a preprocessing step will then add the constraints $K(l(\tau) \leq \mu)$ for all free variables and constants $x_{\tau}^{\mu}$. (Notice that we don't need to do this for bound variablesTbecause the constraints are those in $K\left(l\left(\theta_{x}\right) \leq \nu_{x}\right)$ introduced in the abstraction case.) This avoids rebuilding these same constraints over and over at each occurrence of the same variable. We can also avoid adding the constraints in $K\left(\theta_{x}\right.$ ramified) in the abstraction case as soon as $x$ occurs free in the abstraction $\Gamma$ since then each occurrence of $x$ will produce constraints of the form $K\left(\tau \sqsubseteq \theta_{p}\right)$ Twhere $\tau=\theta_{x}$ Tand this forces $\theta_{x}$ to be ramified by Lemma 2. Still on the chapter of variables Twe can use Theorem 8 to fail right away when trying to type applications of the form $x u$ Twhere $x$ is a variable or a constant that occurs free in $u$.

In the application step $\Gamma$ we add constraints of the form $K\left(\tau_{1}=\tau_{p}^{1}\right) \Gamma \mu_{1}=\mu_{p 1} \Gamma \mu_{p}=\mu_{p 0} \Gamma$ which are equalities between level variables. Instead of representing an equality $\mu=\nu$ as two edges $\mu \stackrel{0}{\leftrightarrow} \nu$ and $\nu \Leftrightarrow \rightarrow$ Cit is more efficient to simply merge the nodes of $\mu$ and $\nu$. This not only decreases the size of the graph $G\left(K_{p}(\sigma)\right) \Gamma$ but also and therefore accelerates the algorithm of Lemma 18 applied on $G\left(K_{\varepsilon}([])\right)$. (In particularTobserve that this correctly identifies all connected components of the first example of Section 5.3 right away.)

In the abstraction step $\Gamma$ we can also dispense with the creation of a variable $y_{\theta_{x}}^{\nu_{x}}$. Observe that what we really need is just $\theta_{x}$ and $\nu_{x} \Gamma$ not $y$. This is only a minor point.

A more important point is that although we examine applications as unary applicationsTit is more profitable to deal with $n$-ary applications in one fell swoop. That is $\Gamma$ observe that any term can be written uniquely as $\lambda x_{1 \epsilon_{1}} \cdot \ldots \cdot \lambda x_{m \epsilon_{m}} \cdot h u_{1} \ldots u_{n} \Gamma$ where $m \geq 0 \Gamma n \geq 0$ and $h$ is not an application. Then we type it (at occurrence $p$ ) by extending $\sigma$ to $\sigma\left[y_{\theta_{x_{1}}}^{\nu_{x_{1}}} / x_{1}, \ldots, y_{\theta_{x_{m}}}^{\nu_{x_{m}}} / x_{m}\right] \Gamma$ typing $h \Gamma u_{1} \Gamma$ $\ldots \Gamma u_{n} \Gamma$ failing if the type of $h$ is not of the form $\tau_{1} \stackrel{\mu_{1}}{\rightarrow} \ldots \tau_{n} \stackrel{\mu_{n}}{\rightarrow} \tau$ Twith $\tau_{i}$ having the same erasure as the type $\tau_{i}^{\prime}$ of $u_{i}$ for each $i \Gamma 1 \leq i \leq n \Gamma$ and then producing $K\left(\theta_{x_{j}}\right.$ ramified) and $\bar{K}\left(l\left(\bar{\theta}_{x_{j}}\right) \leq \nu_{x_{j}}\right)$ for every $1 \leq j \leq m \Gamma K\left(\tau_{i}=\tau_{i}^{\prime}\right) \Gamma \mu_{i}=\mu_{i}^{\prime}$ (where $\mu_{i}^{\prime}$ is the level of $u_{i}$ ) for every $1 \leq i \leq n \Gamma$ plus $\mu_{p}=\mu$ if $m=0$ or $\mu_{p} \geq \nu_{x_{1}}+1 \Gamma \ldots \Gamma \mu_{p} \geq \nu_{x_{m}}+1 \Gamma \mu_{p} \geq \mu$ if $m>0 \Gamma$ where $\mu$ is the level of $h$; and we return $\theta_{x_{1}} \stackrel{\nu_{x_{1}}}{\rightarrow} \ldots \theta_{x_{m}} \stackrel{\nu_{x_{m}}}{\rightarrow} \tau$ as $\tau_{p}(\sigma)$. This cuts drastically on the number of auxiliary level variables that we need $\Gamma$ at least when we have many abstractionsTconstants or variables taking several arguments; indeed $\Gamma$ we don't need any variable for $\left(\lambda x_{2 \epsilon_{2}} \cdot \ldots \cdot \lambda x_{m \epsilon_{m}} \cdot h u_{1} \ldots u_{n}\right) \Gamma$ $\ldots \Gamma\left(\lambda x_{m \epsilon_{m}} \cdot h u_{1} \ldots u_{n}\right) \Gamma\left(h u_{1} \ldots u_{m}\right) \Gamma\left(h u_{1} \ldots u_{m-1}\right) \Gamma$ $\ldots$. Tor $h u_{1}$ any longer.

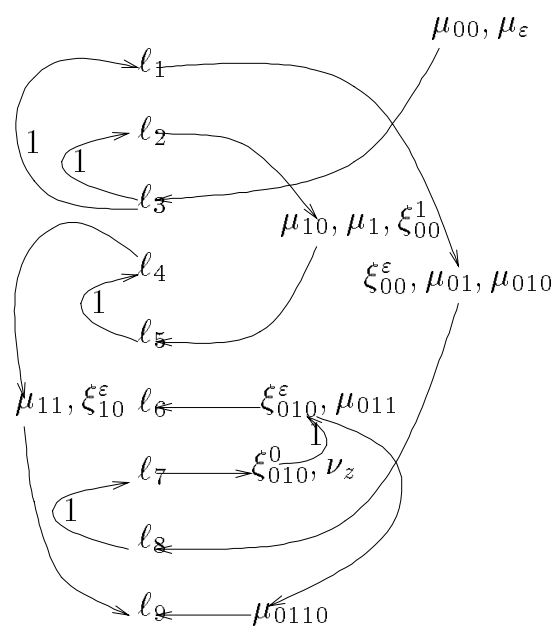

Figure 8: Simplified graph

Returning to the example $a\left(x\left(\lambda z_{\beta} \cdot y\right)\right)(b y)$ of Section $5.3 \Gamma$ and applying the tricks above $\Gamma$ we get the graph of Figure 8 . The main gain comes from identifying nodes that are equated in the application step. (Observe also that this made us merge the two $\Leftrightarrow$ arrows from $\xi_{010}^{\varepsilon}$ to $\xi_{010}^{0}$ Tand from $\mu_{011}$ to $\nu_{z}$.) The trick where we decompose terms as $m$-ary abstractions of $n$-ary applications $\lambda x_{1 \epsilon_{1}} \cdot \ldots \cdot \lambda x_{m \epsilon_{m}} \cdot h u_{1} \ldots u_{n}$ only makes the variable $\mu_{0}$ disappear $\Gamma$ which is not much: this is because there is only one binary application and no $n$-ary abstraction $\Gamma>1$ Tin the example.

We can also improve Algorithm 21 in common cases by doing a small amount of preprocessing $\Gamma$ where we type-check the term in the simple theory of types first $\Gamma$ and at the same time check that in each component $\lambda x_{1 \epsilon_{1}} \cdot \ldots \cdot \lambda x_{m \epsilon_{m}} \cdot h u_{1} \ldots u_{n}$ where $h$ is a constant or a variable $\mathrm{h} h$ does not occur free in $u_{1} \Gamma$ ... Tor $u_{n}$ (the vicious circle principle). Otherwise Tby Theorem 8Ttype-checking fails. If the preprocessing phase does not fail Ithen we apply Algorithm 21 (with the improvements described above). The latter $\Gamma$ now $\Gamma$ cannot fail $\Gamma$ and merely builds a constraint graph $\Gamma$ which we then solve by Lemma 18 .

This preprocessing will catch most untypable instances. However $\Gamma$ notice that it won't catch all of them: there are well-simply-typed terms in normal form that obey the vicious circle principle but cannot be well-typed in the ramified sense. For instance $\Gamma$ consider $\lambda x_{((\beta \rightarrow \beta) \rightarrow \beta) \rightarrow \beta}$. $\lambda y_{\beta \rightarrow \beta} \cdot y\left(x a_{(\beta \stackrel{0}{\rightarrow} \beta) \stackrel{1}{\rightarrow} \beta}\right)\left(x b_{(\beta \stackrel{2}{\rightarrow} \beta) \stackrel{3}{\rightarrow} \beta}\right)$. This term is well-simply-typed of type $\beta \Gamma$ is $\beta \eta$-normal $\Gamma$ obeys the vicious circle principle but is not typable. In- 


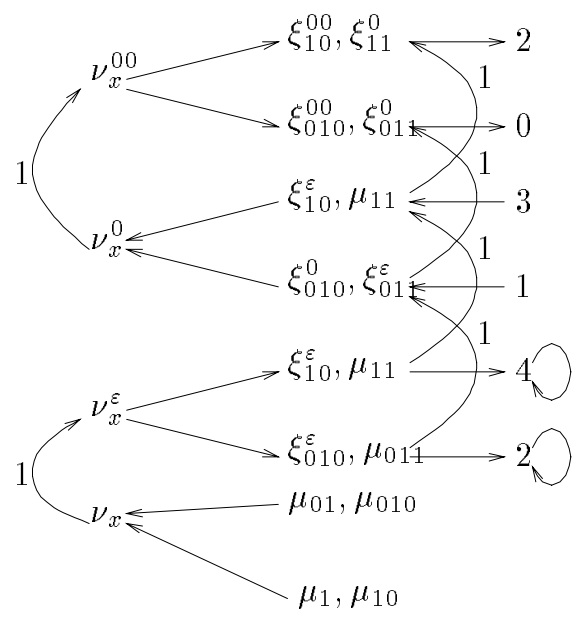

Figure 9: Another untypable term

deed $\Gamma$ the part of the graph that the Algorithm 21 builds corresponding to the subterms $x a$ and $x b$ is shown in Figure 9: check that the path

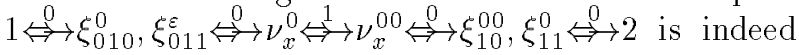
insatisfiable. The reason why this example is not typable is that the types of $a$ and $b$ have no common $\sqsubset-$ lower bound $\Gamma$ so that the same variable $x$ cannot be applied to both $a$ and $b$.

\section{Decidability of Unification}

\subsection{Integer Levels, $\beta \eta$-Equality}

Building on Sections 4 and 5 Twe prove that ramified higher-order ramification with integer levels $(L=\omega)$ is decidable.

First $\Gamma$ we make precise the informal argument of decreasing levels discussed in Section 3.3. We have already said that this informal argument was not quite correct. The main reason why is that it is not so much the levels of free variables that count as the levels that we can get by instantiating these free variables by a unifier and reducing. This justifies the following definition; recall that a substitution is normalized when it maps variables to terms in $\beta$-normal $\eta$-expanded form.

Definition 7 For every $\beta$-normal term $u$ of the form $\lambda y_{\epsilon_{1}} \cdot \ldots \cdot \lambda y_{n_{\epsilon_{n}}} \cdot t$, let the min-level $l(u)$ of $u$ be the least $\ell$ such that $t\left[x_{\tau_{1}}^{\ell_{1}} / y_{1}, \ldots, x_{\tau_{n}}^{\ell_{n}} / y_{n}\right]$ is typable of type $\tau / \ell$ for some type $\tau$, where $E\left(\tau_{1}\right)=\epsilon_{1}, \ldots$, $E\left(\tau_{n}\right)=\epsilon_{n}$.

Lemma 23 For any well-typed normalized substitution $\sigma$, for every variable $F_{\tau}^{\ell}, l(F \sigma) \leq \ell$.
Proof: Since $\sigma$ is well-typed $\Gamma F \sigma$ is typable of some type $\tau^{\prime} / \ell^{\prime}$ with $\tau^{\prime} \sqsubseteq \tau$ and (1) $\ell^{\prime} \leq \ell$. Let's write $\tau^{\prime}$ as $\tau_{1} \stackrel{\ell_{1}}{\rightarrow} \ldots \tau_{n} \stackrel{\ell_{n}}{\rightarrow} b$ Twhere $b$ is a base type. Since $\sigma$ is normalized $\Gamma F \sigma$ must be of the form $\lambda y_{1_{1}} \cdot \ldots \lambda y_{n_{\epsilon_{n}}} \cdot t$; and by rule $(A b s) \Gamma E\left(\tau_{1}\right)=\epsilon_{1} \Gamma \ldots \Gamma E\left(\tau_{n}\right)=\epsilon_{n} \Gamma$ and $t\left[x_{\tau_{1}}^{\ell_{1}} / y_{1}, \ldots, x_{\tau_{n}}^{\ell_{n}} / y_{n}\right]$ is typable of type $b / \ell^{\prime \prime} \Gamma$ with $\ell^{\prime}=$ $\max \left(\ell_{1}+1, \ldots, \ell_{n}+1, \ell^{\prime \prime}\right)$. In particular $\Gamma(2) \ell^{\prime \prime} \leq \ell^{\prime}$. By Definition $7 \Gamma(3) l(F \sigma) \leq \ell^{\prime \prime}$. By $(1) \Gamma(2)$ and $(3)$ it follows that $l(F \sigma)<\ell$.

Let's say that a substitution is weakly well-typed if and only if it maps every variable $x_{\tau}^{\ell}$ to some term $t$ of type $\tau^{\prime} / \ell^{\prime}$ with $E(\tau)=E\left(\tau^{\prime}\right)$. A well-typed substitution imposes moreover $\tau=\tau^{\prime}$ and $\ell=\ell^{\prime}$.

Lemma 24 Let $\sigma$ be an arbitrary weakly well-typed normalized substitution, $F$ a variable, $t=\lambda \overline{y_{n}}$.

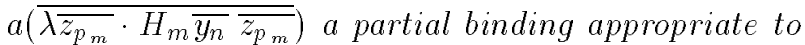
$F$, where $a$ is a constant or a bound variable.

If $\sigma$ unifies $F$ and $t$, then for every $1 \leq i \leq m$, $l\left(H_{i} \sigma\right)<l(F \sigma)$.

Proof: $\quad F \sigma$ is a $\beta$-normal $\eta$-expanded term $\Gamma$ say $\lambda y_{\epsilon_{1}} \cdot \ldots \cdot \lambda y_{\epsilon_{\epsilon_{n}}} \cdot t$. (The abstraction header is the same as that of $t \Gamma$ because both those terms are $\eta$ expanded terms and of the same simple type.)

Since $\sigma$ is normalized $\Gamma$ for each $1 \leq i \leq m \Gamma H_{i} \sigma$ is $\beta$-normal and $\eta$-expanded $\Gamma$ so it is $\lambda y_{1}^{\prime} \cdot \ldots \lambda y_{n}^{\prime}$. $\lambda z_{1}^{\prime} \cdot \ldots \cdot \lambda z_{p_{i}}^{\prime} \cdot t_{i}$ for some $\beta$-normal term $t_{i}$ of type $b_{i}$. Without loss of generality $\Gamma$ we may drop the primes on the bound variables in the header of $H_{i} \sigma$ Tso that $\left(H_{i} \sigma\right) \overline{y_{n}} \overline{z_{p_{i}}}$ is just $t_{i}$.

Since $\sigma$ unifies $F$ with $t \Gamma F \sigma=\beta \eta(t \sigma) \downarrow \Gamma$ and because both sides of the equality are $\beta$-normal and $\eta$-expanded $\Gamma F \sigma=(t \sigma) \downarrow=\lambda \overline{y_{n}} \cdot a\left(\lambda \overline{z_{p_{1}}} \cdot t_{1}\right) \ldots\left(\lambda \overline{z_{p_{m}}}\right.$. $\left.t_{m}\right)$. So $t=a\left(\lambda \overline{z_{p_{1}}} \cdot t_{1}\right) \ldots\left(\lambda \overline{z_{p_{m}}} \cdot t_{m}\right)$. Letting $x_{1} \ell_{\tau_{1}} \Gamma$ $\ldots \Gamma x_{n}^{\ell_{n}}$ be fresh variables with $E\left(\tau_{1}\right)=\epsilon_{1} \Gamma \ldots \Gamma$ $E\left(\tau_{n}\right)=\epsilon_{n}$ Twe have:

$$
\begin{aligned}
& t\left[x_{1} / y_{1}, \ldots, x_{n} / y_{n}\right]= \\
& a^{\prime}\left(\lambda \overline{z_{p_{1}}} \cdot t_{1}\left[x_{1} / y_{1}, \ldots, x_{n} / y_{n}\right]\right) \\
& \quad \ldots\left(\lambda \overline{z_{p_{m}}} \cdot t_{m}\left[x_{1} / y_{1}, \ldots, x_{n} / y_{n}\right]\right)
\end{aligned}
$$

where $a^{\prime}=a\left[x_{1} / y_{1}, \ldots, x_{n} / y_{n}\right]$. (So $a^{\prime}=a$ for imitation bindings $\Gamma$ and $a^{\prime}=x_{j}$ for some $j$ in the case of projection bindings.) In particular $\Gamma l(F \sigma)$ is the least $\ell$ such that $a^{\prime}\left(\lambda \overline{z_{p_{1}}} \cdot t_{1}\left[x_{1} / y_{1}, \ldots, x_{n} / y_{n}\right]\right) \ldots\left(\lambda \overline{z_{p_{m}}}\right.$. $\left.t_{m}\left[x_{1} / y_{1}, \ldots, x_{n} / y_{n}\right]\right)$ is typable of type $\tau / \ell$ for some $\tau$.

Any normalized derivation of the latter must end in $m$ instances of $(A p p) \Gamma$ following one instance of $\left(V a r^{\prime}\right)$ to type $a^{\prime}$. Now $a^{\prime}$ is a variable or constant $\Gamma$ which is assigned some ramified type $\tau_{1}^{\prime} \stackrel{\ell_{1}^{\prime}}{\rightarrow} \ldots \tau_{m}^{\prime} \stackrel{\ell_{m}^{\prime}}{\rightarrow} b$ and some level $\ell^{\prime}$ in the derivation by rule $\left(V a r^{\prime}\right)$ Twith 
(1) $\ell^{\prime}=\ell$ (by rule $(A p p)$ ); since this type is ramified by Lemma $3 \Gamma(2) \ell^{\prime} \geq \max \left(\ell_{1}^{\prime}+1, \ldots, \ell_{m}^{\prime}+1\right)$. On the other hand $\Gamma$ for each $1 \leq i \leq m \Gamma \lambda \overline{z_{p_{i}}}$. $t_{i}\left[x_{1} / y_{1}, \ldots, x_{n} / y_{n}\right]$ must have received the decorated type $\tau_{i}^{\prime} / \ell_{i}^{\prime} \Gamma$ by rule $(A p p)$. To type the latter $\Gamma$ we must have used rule $(A b s) p_{i}$ times $\Gamma$ and $t_{i}\left[x_{1} / y_{1}, \ldots, x_{n} / y_{n}, x_{1 \theta_{1}}^{\prime l_{1}} / z_{1}, \ldots, x_{p_{i \theta_{p_{i}}}}^{l_{p_{p_{i}}} / z_{p_{i}}}\right] \Gamma$

where $x_{j_{\theta_{j}}}^{l_{j}} \Gamma 1 \leq j \leq p_{i} \Gamma$ are fresh variables $\Gamma$ must have been given a (base) type $b_{i}$ and a level $l_{i}^{\prime}$; so $\tau_{i}^{\prime}=$ $\theta_{1} \stackrel{l_{1}}{\rightarrow} \ldots \theta_{p_{i}} \stackrel{\ell_{p_{i}}}{\rightarrow} b_{i}$ and $(3) \ell_{i}^{\prime}=\max \left(l_{1}+1, \ldots, l_{p_{i}}+1, l_{i}^{\prime}\right)$ (by $(A b s))$. And by Definition $7 \Gamma(4) l_{i}^{\prime} \geq l\left(H_{i} \sigma\right)$.

By (1) and (2) $\ell=\ell^{\prime} \geq \ell_{i}^{\prime}+1$. But by (3) $\ell_{i}^{\prime} \geq l_{i}^{\prime} \mathrm{T}$ so $\ell \geq l_{i}^{\prime}+1$ Tand by (4) $\ell \geq l\left(H_{i} \sigma\right)+1$. Since $l(F \sigma)$ is the lowest such $\ell$ Tit follows that $l(F \sigma)>l\left(H_{i} \sigma\right)+1$.

We shall also need the following:

Definition 8 For any simple type $\epsilon$, let $\tau(\epsilon)$ be the ramified type defined by $\tau(b)=b$ for any base type $b$ and $\tau\left(\epsilon_{1} \rightarrow \epsilon_{2}\right)=\tau\left(\epsilon_{1}\right) \stackrel{l\left(\tau\left(\epsilon_{1}\right)\right)}{\rightarrow} \tau\left(\epsilon_{2}\right)$.

Lemma 25 For any simple type $\epsilon, \tau(\epsilon)$ is a ramified type $\tau$ of minimal level such that $E(\tau)=\epsilon$.

Proof: That $\tau(\epsilon)$ is a ramified type follows from the definition. By an easy structural induction on $\epsilon \mathrm{T}$ $E(\tau(\epsilon))=\epsilon$. Finally let $\tau$ be another type such that $E(\tau)=\epsilon \Gamma$ then we claim that $l(\tau) \geq l(\tau(\epsilon))$. This is proved by structural induction on $\epsilon$. If $\epsilon$ is a base type $\Gamma$ then $l(\tau)=0=l(\tau(\epsilon))$. And if $\epsilon$ is of the form $\epsilon_{1} \rightarrow \epsilon_{2}$ Tthen $\tau$ is of the form $\tau_{1} \stackrel{\ell_{1}}{\rightarrow} \tau_{2}$ Twhere by induction hypothesis (1) $l\left(\tau_{1}\right) \geq l\left(\tau\left(\epsilon_{1}\right)\right) \Gamma(2) l\left(\tau_{2}\right) \geq$ $l\left(\tau\left(\epsilon_{2}\right)\right) \Gamma$ and because $\tau$ is ramified $(3) \ell_{1} \geq l\left(\tau_{1}\right)$. By (1) and (3) $\Gamma$ it follows (4) $\ell_{1} \geq l\left(\tau\left(\epsilon_{1}\right)\right)$. And $l(\tau)=\max \left(\ell_{1}+1, l\left(\tau_{2}\right)\right) \geq \max \left(l\left(\tau\left(\bar{\epsilon}_{1}\right)\right)+1, l\left(\tau_{2}\right)\right)$ (by (4)) $\geq \max \left(l\left(\tau\left(\epsilon_{1}\right)\right)+1, \bar{l}\left(\tau\left(\epsilon_{2}\right)\right)\right)($ by $(2))=l(\tau(\epsilon))$.

We are now able to formulate our algorithm for ramified higher-order unification with integer levels. Recall that the input is a multiset $M$ of unordered pairs of simply-typed terms in $\beta$-normal form. For each free variable $x$ Twe estimate an upper bound $\ell$ on the possible $l(x \sigma)$ for any unifier $\sigma$. To map each variable to this upper bound $\Gamma$ we use the following trick: we store $\ell$ as the level of the variable $\Gamma$ i.e. Twe write $x$ as $x_{\tau}^{\ell} \Gamma$ for some $\tau$. This is certainly consistent with the usual meaning of levelsT by Lemma 23 . Lemma 25 will allow us to find $\tau$. The only difficulty is that a unifier $\sigma$ need not assign a term of level at most $\ell$ to these new variablesThence the notion of weakly well-typed substitutions that we have introduced just before Lemma 24 .
We use Lemma 24 and Lemma 25 to refine Definition 3:

Definition 9 Let $\epsilon$ be a simple type, which we write $\epsilon_{1} \rightarrow \ldots \epsilon_{n} \rightarrow b$ with $b$ a base type, $\ell$ be an integer level, and a be a variable or constant of simple type $\epsilon_{1}^{\prime} \rightarrow \ldots \epsilon_{m}^{\prime} \rightarrow b$.

For each $1 \leq i \leq m$, let's write $\epsilon_{i}^{\prime}$ as $\epsilon_{1}^{\prime \prime i} \rightarrow$ $\ldots \epsilon_{p_{i}}^{\prime \prime i} \rightarrow b_{i}$, where $b_{i}$ is a base type. Assume that:

$$
\text { (A) } \ell>l\left(\tau\left(\epsilon_{1} \rightarrow \ldots \epsilon_{n} \rightarrow \epsilon^{\prime \prime}\right)\right)
$$

for each $1 \leq i \leq m$.

The set $\bar{B}(\epsilon, \bar{a}, \ell)$ of partial bindings of type $\epsilon$, head $a$, and level $\ell$, is the set of terms of the form:

$$
\begin{aligned}
& \left.\lambda y_{\epsilon_{\epsilon_{1}}} \cdot \ldots \cdot \lambda y_{\epsilon_{\epsilon_{n}}} \cdot{ }^{a\left(\lambda \overline{z_{p_{1}}^{1}}\right.} \cdot H_{1} \overline{y_{n}} z_{p_{1}}^{1}\right) \ldots\left(\lambda \overline{z_{p_{m}}^{m}} \cdot H_{m} \overline{y_{n}} \overline{z_{p_{m}}^{m}}\right)
\end{aligned}
$$

where:

1. for every $1 \leq i \leq m, 1 \leq j \leq p_{i}$, $z_{j}^{i}$ is a variable $\left(z_{j}^{i}\right)_{\epsilon_{j}^{\prime \prime i}}$;

2. for every $1 \leq i \leq m, H_{i}$ is a variable $\left(H_{i}\right)_{\tau\left(\epsilon_{1} \rightarrow \ldots \epsilon_{n} \rightarrow \epsilon_{1}^{\prime \prime i} \rightarrow \ldots \epsilon_{p_{i}}^{\prime \prime i} \rightarrow b_{i}\right)}^{\ell-1}$;

The set $B(F, a)$ of partial bindings head a appropriate to $F_{\tau}^{\ell}$ is $B(E(\tau), a, \ell)$.

We let projection and imitation bindings be as in Definition $3 \Gamma$ and keep the same notation $\Gamma$ so that we can use the rules of Figure 2 for ramified preunification. Notice that an essential difference is that projection bindings only exist if condition $(A)$ above is satisfied. Indeed $\Gamma$ without condition $(A) \Gamma$ there could be no variable $H_{i}$ of ramified type of erasure $\epsilon_{1} \rightarrow \ldots \epsilon_{n} \rightarrow \epsilon^{\prime \prime}{ }_{1}^{i} \rightarrow \ldots \epsilon_{p_{i}}^{\prime \prime} \rightarrow b_{i}$ and level at most $\ell \Leftrightarrow 1$ Tby Lemma 25 .

Algorithm 26 Let $N$ be an auxiliary function defined as follows: for any such multiset $S, N(S)$ applies (Delete), (Decomp), (Bind) on $S$ until this is no longer possible, and returns the resulting multiset.

1. Initialize $S$ to $N(M)$.

2. While $S$ is not in solved form, do:

(a) pick any flexible-rigid pair $\left\langle\lambda \overline{x_{k}} \cdot F_{\tau}^{\ell} \overline{u_{n}}, \lambda \overline{x_{k}}\right.$. $\left.a \overline{v_{m}}\right\rangle$ in $S$, with $\ell$ satisfying condition $(A)$, namely $\ell>l\left(\tau\left(\epsilon_{i}\right)\right)+1$ for each $x_{i_{i}}, 1 \leq$ $i \leq k$, and $\ell>l\left(\tau\left(\epsilon^{\prime}\right)\right)$ where $\epsilon^{\prime}$ is the simple type of $a$;

(b) if there is none, then fail; 
(c) otherwise, apply (Imitate) or (Project) non-deterministically on it, getting a new multiset $S_{a}$;

(d) let $S$ be $N\left(S_{a}\right)$, and loop.

3. Let $\sigma$ the substitution represented by $S$, restricted to the free variables of $M$. For every variable $x_{\tau}^{\ell}$ in dom $\sigma$, check that $(x \sigma \zeta) \downarrow$ is typable of type $\tau / \ell$. If so, return $\sigma$, otherwise fail.

Step 3 is accomplished by using Algorithm 21 and Lemma 18 Tand testing for each $x \tau^{\ell} \in \operatorname{dom} \sigma$ whether $K\left(s: \tau_{s} / \mu_{s}\right) \cup K\left(\tau=\tau_{s}\right) \cup\left\{\mu_{s}=\ell\right\}$ is satisfiable $\mathrm{I}$ where $s=(x \sigma \zeta) \downarrow$.

Furthermore Tthe meaning of "pick" above denotes an arbitrary choice: picking another flexible-rigid pair does not affect soundness or completeness - although it may affect the efficiency of the algorithm. On the contrary the rules to apply to the given flexible-rigid pair are applied non-deterministicallyГi.e. by backtracking for example.

Theorem 27 (Termination) Algorithm 26 terminates.

Proof: We first claim that $N$ is well-defined $\Gamma$ i.e. that any sequence of applications of (Delete) $\Gamma($ Decomp $\Gamma$ (Bind) on $S$ must terminate. Let $s(S)$ be defined as $\sum_{c \in S} s(c) \Gamma$ where $s\left\langle u_{i}, v_{i}\right\rangle=s\left(u_{i}\right)+s\left(v_{i}\right) \Gamma$ $s(x)=1$ for any variable or constant $x \Gamma s(u v)=$ $s(u)+s(v)+1$ and $s\left(\lambda x_{\epsilon} \cdot u\right)=s(u)$. Let $\#(S)$ be defined as the number of solved pairs $\langle\eta[x], \eta[s]\rangle$ in $S$ (or equivalently $\mathrm{T}$ as the number of solved variables). Then (Delete) does not increase \#(S) and decreases $s(S)$ by $2 s(u)$. (Decomp) leaves \#(S) constant and decreases $s(S)$ by (see Figure 2 for notations) $\left(s(a)+\sum_{i=0}^{n}\left(s\left(u_{i}\right)+1\right)+\sum_{i=0}^{n}\left(s\left(v_{i}\right)+1\right)\right) \Leftrightarrow$ $\left(\sum_{i=0}^{n} u_{i}+\sum_{i=0}^{n} v_{i}\right)=2 n+1>0$. And in the case of (Bind) (see Figure 2 for notations) Tsince $F$ is assumed to be free in $S^{\prime} \Gamma F$ is not solved before applying the rule; but $F$ is solved after applying it. Moreover $\Gamma$ any other variable $G$ that occurred in a solved pair $\langle\eta[G], \eta[t]\rangle$ before occurs only in the pair $\left\langle\eta[G],\left(\eta[t]\left[\lambda \overline{x_{k}} \cdot v / F\right]\right) \downarrow\right\rangle$ afterwards $\Gamma$ which is solved because $G$ does not occur in $v$. So \#(S) decreases strictly in this case.

We now claim that the loop in step 2 of Algorithm 26 can only be traversed finitely many times. Let $\mathcal{L}(S)$ be defined as the set of unsolved variables in $N(S)$. We order such sets $A$ by letting $\alpha(A)$ denote $\sum_{x_{\tau}^{\ell} \in A} \omega^{\ell} \Gamma$ where the sum is the natural sum of ordinals (i.e. the summands are first sorted in decreasing order); this is akin to a multiset extension of the ordering on levels of variables [Der87].
ThenTany use of (Imitate) or (Project) on STfollowed by a call to the procedure $N$ Twill apply (Bind) on $F$ and (Decomp) on the pair under consideration. That is $\Gamma$ step 2.(b) transforms $S$ into a new multiset $S_{a}$ where $F$ is solved $\Gamma$ whereas $F$ was not solved in $S$ T by the side-conditions on (Imitate) Tresp. (Project). Moreover $\Gamma$ all the solved free variables in $S$ remain solved in $S_{a}$. So $\mathcal{L}\left(S_{a}\right)$ is obtained from $\mathcal{L}(S)$ by replacing the unsolved variable $F$ at level $\ell$ by finitely many unsolved variables $H_{i} \Gamma 1 \leq i \leq m \Gamma$ with levels $\ell \Leftrightarrow 1$ Tand possibly erasing some other unsolved variables. Therefore $\alpha\left(\mathcal{L}\left(S_{a}\right)\right)<\alpha(\mathcal{L}(S))$. Since the ordering on ordinals is well-foundedГstep 2 can only be applied finitely many times.

FinallyTstep 3 terminates because Algorithm 21 and the algorithm of Lemma 18 terminate.

Theorem 28 (Soundness) For any $\sigma$ returned by Algorithm 26, $(\sigma \zeta) \downarrow$ is a ramified unifier of $M$.

Proof: By the soundness of simply-typed unificationT the substitution $\varsigma$ represented by the multiset $S$ of step 3 is a well-simply-typed pre-unifier of $M$. That is $\Gamma(\varsigma \zeta) \downarrow$ is a unifier of $M$. Letting $\sigma$ be $\left.\varsigma\right|_{\operatorname{FVC}(M)} \Gamma$ therefore $\Gamma(\sigma \zeta) \downarrow$ is also a unifier of $M$. Moreover $\Gamma$ it is well-typed by step 3 and the correctness of Algorithm 21 and of the algorithm of Lemma 18.

Theorem 29 (Completeness) For any ramified unifier $\varsigma$ of $M$, there is a computation branch of Algorithm 26 that returns a substitution $\sigma$ such that $\varsigma$ is an instance of $\sigma$-i.e., there is a substitution $\sigma^{\prime}$ such that $\varsigma={ }_{\beta \eta} \sigma \sigma^{\prime}$.

Proof: Since $\varsigma$ is a ramified unifier of $M \Gamma$ it is also a well-simply-typed unifier of $M$. By the completeness of Huet's algorithm $\Gamma$ with the strategy applying (Bind) $\Gamma($ Decomp) and (Delete) eagerly $\Gamma \varsigma$ is an instance (modulo $\beta \eta$ ) of some substitution found by applying steps 1 and 2 of Algorithm 26 एwith the exception that we don't check condition $(A)$. More formally there is a finite sequence of unification problems $S_{0} \Gamma S_{1} \Gamma \ldots \Gamma S_{p} \Gamma p \geq 0 \Gamma$ such that $S_{0}=N(M)$ (step 1) $\Gamma$ and for every $1 \leq j \leq p \Gamma S_{i}=N\left(S_{a} i-1\right) \Gamma$ where $S_{a-1}$ is obtained from $S_{i-1}$ by applying (Imitate) or (Project) on some arbitrary flexible-rigid pair of $S_{i-1}$. Moreover $\Gamma \varsigma$ unifies every $S_{j} \Gamma 0 \leq j \leq p \Gamma$ in the simply-typed sense. In particularT $\varsigma$ is weakly well-typed in the ramified sense.

We claim that for every variable $x_{\tau}^{\ell}$ free in any $S_{j} \Gamma$ $0 \leq j \leq p \Gamma l(x \varsigma) \leq \ell$. We prove the claim by induction on $j$. This is indeed true for all variables free in $S_{0} \Gamma$ since $\varsigma$ is a well-typed (ramified) unifier of $M$ and by Lemma 23 . Then $\mathrm{T}$ for all variables free in 
$S_{j}$ but not in $S_{j-1}$ Tsuch variables are variables $H_{i} \Gamma$ $1 \leq i \leq m$ Tcoming from a partial binding appropriate to some variable $F$ free in $S_{j-1}$; so by Lemma $24 \Gamma$ $l\left(H_{i} \varsigma\right)<l(F \varsigma)$. By induction hypothesisTand letting $\ell$ be the level annotating $F$ Twe have $l(F \varsigma) \leq \ell$ Thence $l\left(H_{i} \varsigma\right)<\ell$. It follows that $l\left(H_{i} \varsigma\right) \leq \ell \Leftrightarrow 1$ Twhere $\ell \Leftrightarrow 1$ is precisely the level decorating $H_{i}$ Tfor each $1 \leq i \leq m$.

Therefore the finite sequence of unification problems verifies condition $(A)$ at each turn through step 2 Tby Lemma 25 . Therefore Tthe step 2 loop terminates successfully. Finally by Lemma 13 and the correctness of Algorithm 21 and the algorithm of Lemma $18 \Gamma$ step 3 also terminates successfully. Since $\varsigma$ unifies $S_{p} \Gamma$ i.e. the $S$ that we find in step 3 Tit is by construction an instance of the $\sigma$ returned by the algorithm.

\section{Corollary 30}

Ramified higher-order unification with integer levels is decidable.

Proof: By Theorems 27Г28 and 29.

Algorithm 26 is not incremental as it standsTin that we cannot unify $\left\{\left|\left\langle u_{1}, v_{1}\right\rangle,\left\langle u_{2}, v_{2}\right\rangle\right|\right\}$ by first running it on the pair $\left\langle u_{1}, v_{1}\right\rangle \Gamma$ choosing one of the answers $\sigma$ I and then unifying $\left\langle u_{2} \sigma, v_{2} \sigma\right\rangle$. Indeed $\Gamma u_{2} \sigma$ and $v_{2} \sigma$ may contain variables $H_{i}$ invented by the (Imitate) and (Project) steps $\Gamma$ and the levels of these variables are mere codings of upper bounds on the min-level $l\left(H_{i} \sigma\right)$ Tnot on the levels of terms to subtitute for these variables.

Incremental unification algorithms are useful in automated theorem proving and elsewhere $\mathrm{C}$ and the following modification to Algorithm 26 makes it incremental: we separate the set of free variables in two disjoint sets. Variables $x_{\tau}^{\ell}$ from the first set can only be instantiated by terms of level at most $\ell \Gamma$ while variables $y_{\tau}^{\ell}$ from the second set are used as fresh variables in (Imitate) and (Project) Tand can only be instantiated by terms of min-level at most $\ell$. Algorithm 26 is left unchanged.

\section{$6.2 \beta$-Equality}

It is also interesting to consider unification modulo $\beta \Gamma$ i.e. without the $\eta$ rule. This is in particular important in the case of ramified type theory which is so intensional in nature [Cop71]. We sketch here why these cases are still decidable.

Just dropping the $\eta$-rule entails that we cannot use the rules of Figure 2 any longer. Instead $\Gamma$ we have to use Huet's method for $\beta$-unification as underlying simply-typed unification procedure [Hue 75]. This procedure is a bit more complicated Tbecause it cannot use $\eta$-expanded forms any longer. In any flexible- rigid pair $\left\langle\lambda \overline{x_{k}} \cdot F_{\tau}^{\ell} \overline{u_{n}}, \lambda \overline{x_{k^{\prime}}} \cdot a \overline{v_{m}}\right\rangle \Gamma k$ is not necessarily equal to $k^{\prime}$ as before $\Gamma$ and we have to adjust arities before imitating or projecting; that is $\Gamma$ we must have $k \leq k^{\prime}$ Tand $F$ must be mapped to some term of the form $\lambda x_{k+1} \cdot \ldots \cdot \lambda x_{j} \cdot u$ Twhere $u$ is a suitable partial binding and $k \leq j \leq k^{\prime}$. Such partial bindings are of the form $\lambda \overline{y_{n}} \cdot a^{\prime}\left(\overline{H_{1}} \overline{y_{n}}\right) \ldots\left(H_{m} \overline{y_{n}}\right) \Gamma$ where $a^{\prime}=a$ or $a^{\prime}$ is a bound variable.

Another variant is to drop the $\eta$-rule and choose a weaker notion of $\alpha$-equivalence; recall that we were forced to choose such a lax notion of $\alpha$-equivalence to be consistent with $\eta$-equality. Consider therefore the language consisting of variables and constants $x_{\tau}^{\ell} \Gamma$ applications $u v \Gamma$ and abstractions $\lambda x_{\tau}^{\ell} \cdot u \Gamma$ with conversion rules:

$$
\begin{array}{ll}
\left(\alpha_{w}\right) & \left(\lambda x_{\tau}^{\ell} \cdot u\right)=\left(\lambda y_{\tau}^{\ell} \cdot u[y / x]\right) \\
(\beta) & (\lambda x \cdot u) v \rightarrow u[v / x]
\end{array}
$$

where $y$ is not free or bound in $u$ in the $\alpha_{w}$ rule.

The typing rules are unchanged but for abstractions:

$$
\frac{u: \tau_{2} / \ell_{2}}{\lambda x_{\tau_{1}}^{\ell_{1}} \cdot u: \tau_{1} \stackrel{\ell_{1}}{\rightarrow} \tau_{2} / \max \left(\ell_{1}+1, \ell_{2}\right)}
$$

Almost all results of this paper are unchanged $\Gamma$ then. Theorem 6 on normal type derivations still holdsTand is in fact simpler to prove than before. Theorem 8 (the vicious circle principle) is unchanged. Subject reduction (Theorem 10) also holds in this case $\Gamma$ and is also a bit simpler to prove. Lemma 11 on erasing levels trivially holds. And provided that we replace " $\beta \eta$-equivalent" by " $\beta$-equivalent" $\Gamma$ Theorem 15 on how to test whether a term has a ramified well-typed instance also holds.

The results of Section 5 had nothing to do with $\lambda$ conversion and therefore still apply. The only real things that change are the notions of Section 6.1. The min-level $l(u)$ of a $\beta$-normal term $u$ of the form $\lambda y_{1} \tau_{1}^{\ell_{1}} \cdot \ldots \cdot \lambda y_{n} \ell_{\tau_{n}}^{\ell_{n}} \cdot t$ is now defined as the level of $t \Gamma$ i.e. the least $\ell$ such that $t: \tau / \ell$ is derivable for some $\tau$. The analogues of Lemmas 23 and 24 then hold provided that by normalized substitution we understand substitution mapping variables to $\beta$-normal terms $\Gamma$ not necessarily $\eta$-expanded. It follows that Algorithm 26 $\mathrm{T}$ with the appropriate notions of partial bindings $\Gamma$ is a terminating $\Gamma$ sound and complete algorithm for $\beta$-unification with weak $\alpha$-conversion.

\section{$7 \quad$ Logic}

Unification is a basic component of automated theorem proving. But in which system of logic? The answer to this question is not so easy as it may seem $\Gamma$ and we discuss several possible approaches. 


$$
\begin{array}{cc}
\frac{\Gamma \triangleright F \triangleright, \Delta}{\Gamma, F \triangleright, \Delta}(A x) \\
\frac{\Gamma, \neg F \triangleright \Delta}{\Gamma}(\neg L) & \frac{\Gamma, F \triangleright \Delta}{\Gamma \triangleright \neg F, \Delta}(\neg R) \\
\frac{\Gamma, F, G \triangleright \Delta}{\Gamma, F \wedge G \triangleright \Delta}(\wedge L) & \frac{\Gamma \triangleright F, \Delta \quad \Gamma \triangleright G, \Delta}{\Gamma \triangleright F \wedge G, \Delta}(\wedge R) \\
\frac{\Gamma,(F[t / x]) \downarrow \beta \eta \triangleright \Delta \quad t: \tau / \ell}{\Gamma, \forall x_{\tau}^{\ell} \cdot F \triangleright \Delta}(\forall L) & \frac{\Gamma \triangleright F\left[y_{\tau}^{\ell} / x\right], \Delta \quad y \notin \mathrm{FVC}(\Gamma, \Delta)}{\Gamma \triangleright \forall x_{\tau}^{\ell} \cdot F, \Delta}
\end{array}
$$

Figure 10: Ramified deduction rules

\subsection{Setting Up A Deduction System}

Ramified type theory gives rise to a system of logic that we call ramified higher-order logic. Due to the choices that we have madeTthis logic will have cumulative levels and be weakly extensional (the $\eta$ rule) $\Gamma$ but we may as well choose more intensional logics (see Section 6.2. The language of the logic enables us to build formulasTfor instance by including operators for negation $\neg \Gamma$ conjunction $\wedge$ and universal quantification $\forall$. Universal quantifications must exist at all types $\tau$ and at all levels $\ell: \forall x_{\tau}^{\ell} \cdot F$ means that $F$ holds of all objects of type $\tau$ and level $\ell$ (or lower).

We adopt for instance the Gentzen-style deduction system of Figure 10. A sequent is any expression of the form $\Gamma \triangleright \Delta \Gamma$ where $\Gamma \Gamma \Delta$ are finite sets of formulae $F \Gamma G$ Tetc. The quantifier rules $(\forall L)$ and $(\forall R)$ are special compared to the corresponding rules in simple type theory in that they not only enforce that $t$ (in $(\forall L))$ or $y$ (in $(\forall R)$ ) have the correct type $\Gamma$ but also all the correct levels.

To get a tableau system from the latter Twe interpret all these rules bottom-up [Fit90]. The $(\forall L)$ rule needs to guess a term $t$ : we represent this term $t$ by a metavariable $x_{\tau}^{\ell}$ (i.e.Ta free variable). The real value of $t$ will be found by instantiating $x$ when we try to close a pathГi.e. to conclude that the current sequent is an instance of $(A x)$ : this involves finding one formula on the left and one formula on the right of later sequent that can be unified. This much is the rationale behind our definition of ramified unificationTand in particular of well-typed substitutionsTin Section 3. The $(\forall L)$ rule needs to introduce a fresh variable $y_{\tau}^{\ell}$ that should never be instantiated $\Gamma$ i.e. that should be treated as a constant: to represent thisTwe may do as Kohlhase in simple type theory [Koh95] $\Gamma$ and manage a database of instantiable variablesTof non-instantiable variables $\Gamma$ and of dependencies between them. (We may also dispense with the $\lambda$-headers in Gallier and Snyder's unification rules by introducing the third class of bound variablesTas does Kohlhase.)

The most natural way to represent logical operators in $\lambda_{\alpha}^{\beta}$ is to make them constants of the language $\Gamma$ i.e. we create negations $\neg^{\ell}: o \stackrel{\ell}{\rightarrow} o / \ell+1$ C conjunctions $\wedge^{\ell}: o \stackrel{\ell}{\rightarrow} \circ \stackrel{\ell}{\rightarrow} o / \ell+1$ at all levels $\ell \in L \Gamma$ and universal quantifiers $\Pi^{\ell^{\prime}+1} \quad$ for all types $\tau$ and all levels $\ell, \ell^{\prime} \in L$ with $\ell^{\prime} \stackrel{(\tau \rightarrow 0) \stackrel{\ell}{\rightarrow} \ell+1}{\rightarrow}$. Then $\Gamma$ we omit type and level annotations when they are not strictly necessary. Moreover $\Gamma$ we write $F \wedge G$ instead of $\wedge F G$ Twe define $F \vee G$ as $\neg(\neg F \wedge \neg G) \Gamma F \Rightarrow G$ as $\neg F \vee G$. Finally write $\forall x_{\tau}^{\ell} \cdot F$ for $\Pi_{\left(\tau \stackrel{\ell^{\prime}+1}{\ell^{\prime}} \stackrel{\ell^{\prime}}{\rightarrow} 0\right.}\left(\lambda y_{\epsilon} \cdot F\right)$ Twhere $\epsilon=E(\tau)$ and $\ell^{\prime}$ is such that $F\left[x_{\tau}^{\ell} / y\right]: o / \ell^{\prime}$ is derivable.

But we face a serious problem There $\Gamma$ as this encoding cannot represent enough formulas. This is Theorem 8 on the vicious circle principle: in a formula $F \wedge G$ Tneither $F$ nor $G$ can contain any conjunctions $\Gamma$ for example. We therefore need to relax the typing conditions on constant operators.

\subsection{Adding Operators}

The most natural way to do this is to enrich the $\lambda_{\alpha}^{\beta}$ languages with operators $\Gamma$ aking to the function symbols of first-order logic. IntuitivelyT an operator $f$ would be such that $f\left(u_{1}, \ldots, u_{m}\right)$ would be at level $\max \left(\ell_{1}, \ldots, \ell_{m}\right)$ when $u_{i}$ is at level $i$ for each $1 \leq i \leq m$. We can then encode the logical connectives and quantifiers as operators $\Gamma$ and the problem above disappears.

More formally we enrich the languages $\lambda_{\alpha}^{\beta}$ with operators $f$ Teach given with a unique signature $\tau_{1} \times$ $\ldots \tau_{m} \Rightarrow \tau$. The terms are either variables $x_{\tau}^{\ell} \Gamma$ ap- 
plications $u v$ Tabstractions $\lambda x_{\epsilon} \cdot u$ or algebraic terms $f\left(u_{1}, \ldots, u_{m}\right) \Gamma$ where $f$ is an operator. The typing rules are as in Figure 1 pplus the additional rule:

$$
\begin{aligned}
& u_{1}: \tau_{1} / \ell_{1} \quad \ldots \quad u_{m}: \tau_{m} / \ell_{m} \\
& \frac{f \text { of signature } \tau_{1} \times \ldots \tau_{m} \Rightarrow \tau}{f\left(u_{1}, \ldots, u_{m}\right): \tau / \max \left(l(\tau), \ell_{1}, \ldots, \ell_{m}\right)}(A l g)
\end{aligned}
$$

The adaptation of Huet's procedure to operators is straightforward: just consider an algebraic term $f\left(u_{1}, \ldots, u_{m}\right)$ as a simply-typed term $f u_{1} \ldots u_{m} \Gamma$ where $f$ is now considered a constant. The theory then goes throughTuntil Lemma 24 Twhere we cannot guarantee any longer that partial bindings must produce fresh variables of strictly lower min-levels. And indeed:

Theorem 31 Unification in the ramified theory of types with operators is undecidable. This holds even at the second order, with integer levels, with only one binary operator $g$ of signature $\tau \times \tau \Rightarrow \tau$ and two constants $a, b$ of type $\tau$.

Proof: This is just Goldfarb's proof [Gol81]. Let $\tau$ be a base type $\Gamma a$ and $b$ be of type $\tau / 0$. The operator $g$ is used as a pairing operator; define $\overline{0} t=t \mathrm{~T}$ $\overline{n+1} t=g(a, \bar{n} t)$ for every integer $n$ Tthen the game is to reencode Hilbert's tenth problem as a unification problem. For this $\Gamma$ we only need to produce unification problems of the form $(0)\langle\overline{1}(F a), F(\overline{1} a)\rangle$ where $F$ is a variable of type $\tau \stackrel{0}{\rightarrow} \tau / 1 \Gamma(1)\left\langle F_{1}\left(F_{2} a\right), F_{3} a\right\rangle \Gamma$ where $F_{1} \Gamma F_{2} \Gamma F_{3}$ are variables of type $\tau \stackrel{0}{\rightarrow} \tau / 1 \Gamma(2)$ $\left\langle d_{1}, e_{1}\right\rangle,\left\langle d_{2}, e_{2}\right\rangle$ where:

$$
\begin{aligned}
& d_{1}=G a b\left(g\left(g\left(F_{3} a, F_{2} b\right), a\right)\right) \\
& e_{1}=g\left(g(a, b), G\left(F_{1} a\right)(\overline{1} b) a\right) \\
& d_{2}=G b a\left(g\left(g\left(F_{3} b, F_{2} a\right), a\right)\right) \\
& e_{2}=g\left(g(b, a), G\left(F_{1} b\right)(\overline{1} a) a\right)
\end{aligned}
$$

$F_{1} \Gamma F_{2} \Gamma F_{3}$ are variables of type $\tau \stackrel{0}{\rightarrow} \tau / 1 \Gamma$ and $G$ is a variable of type $\tau \stackrel{0}{\rightarrow} \tau \stackrel{0}{\rightarrow} \tau \stackrel{0}{\rightarrow} \tau / 1$.

Problems of type (0) encode the type of integers that any well-simply-typed unifier $\sigma$ of such problems must map $F$ to $\lambda x_{\tau} \cdot \bar{n} x$ T for some $n \in \mathbb{N}$. But we have:

$$
\begin{gathered}
y_{\tau}^{0}: \tau / 0 \\
\vdots \\
\bar{n} y_{\tau}^{0}: \tau / 0 \\
\lambda x_{\tau} \cdot \bar{n} x: \tau \stackrel{0}{\rightarrow} \tau / 1
\end{gathered}
$$

so $\left.\sigma\right|_{\{F\}}$ is well-typed (in the ramified sense).

Problems of type (1) encode addition $\Gamma$ in that any unifier $\sigma$ mapping $F_{i}$ to $\lambda x_{\tau} \cdot \overline{n_{i}} x \Gamma 1<i<3 \Gamma$ must be such that $n_{1}+n_{2}=n_{3}$. Because Goldfarb's encoding adds problems of type $(0)$ for each of $F_{1} \Gamma F_{2} \Gamma F_{3} \Gamma$ $\left.\sigma\right|_{\left\{F_{1}, F_{2}, F_{3}\right\}}$ is well-typed.

And problems of type (2) encode multiplication $\Gamma$ in that any unifier mapping $F_{i}$ to $\lambda x_{\tau}$. $\overline{n_{i}} x \Gamma 1 \leq i \leq 3 \Gamma$ must be such that $n_{1} \cdot n_{2}=$ $n_{3}$. Such unifiers then map $G$ to $\lambda x_{\tau}$. $\lambda y_{\tau} \cdot \lambda z_{\tau} \cdot g\left(t_{0} x y, g\left(t_{1} x y, \ldots, g\left(t_{n-1} x y, z\right) \ldots\right)\right)$ Twhere $t_{i} x y=g\left(\overline{i . n_{1}} x, \bar{i} y\right)$. This term has ramified type $\tau \stackrel{0}{\rightarrow} \tau \stackrel{0}{\rightarrow} \tau \stackrel{0}{\rightarrow} \tau / 1$ Tand because Goldfarb's encoding adds problems of type $(0)$ for each of $F_{1} \Gamma \quad F_{2} \Gamma \quad F_{3} \Gamma$ $\left.\sigma\right|_{\left\{F_{1}, F_{2}, F_{3}, G\right\}}$ is well-typed.

In conclusion $\Gamma$ any simply-typed unifier of Goldfarb's problem is also a ramified well-typed unifier of the same problem. The converse is trivial. Therefore Goldfarb's problem also encodes Hilbert's tenth problem as a unification problem in ramified type theory with the operator $g$.

Note that the role of operator $g$ in Theorem 31 is played in logic $\Gamma$ for instance $\Gamma$ by conjunction $\wedge$ (of signature $o \times o \Rightarrow o$ ) Tso undecidability seems unescapable.

Of course $\Gamma$ we still have a pre-unification procedure for this system: this is mostly the same algorithm as before $\Gamma$ except that imitating on a term of the form $\lambda \overline{u_{k}} \cdot f\left(v_{1}, \ldots, v_{i}\right) v_{i+1} \ldots v_{m}$ produces fresh variables with identicalTnot lower min-levels.

\section{Encoding Subsystems of Arithmetic}

The Reverse Mathematics programme [Sim85] aimed at finding the weakest natural systems of logic that allow us to prove several important theorems of mathematics. It turned out that Talthough first-order arithmetic is not always enough to prove even combinatorial theorems $\Gamma$ we usually do not need the full power of even second-order arithmetic to prove more involved theorems like the Bolzano-Weierstrass theorem or Kruskal's Lemma.

Let's consider $\mathrm{ACA}_{0}$ and $\Pi_{k}^{1}-\mathrm{CA}_{0}$ Tfor every $k \geq 0$. These are subsystems of second-order arithmetic constructed as follows. The language is built on a set of first-order variables $x \Gamma y \Gamma z \Gamma \ldots$ denoting naturals $\Gamma$ and set variables $X Г Y \Gamma Z \Gamma \ldots$ denoting sets of naturals. Terms $s \Gamma t \Gamma \ldots$ are built from first-order variables using the function symbols + (addition $\Gamma$ binary) $\Gamma *$ (multiplicationTbinary) $\Gamma s$ (successor $\Gamma u n a r y) \Gamma$ and the constant 0 . Atomic formulas are of the form $s<t \Gamma$ $s=t \Gamma$ and $s \in X \Gamma$ where $X$ is a set variable. Formulas are built from atomic formulas using negation $\neg$ Conjunction $\wedge$ first-order quantification $\forall_{1} x$. and second-order quantification $\forall_{2} X$. We use $\neq \Gamma \leq \Gamma \vee \Gamma$ $\Rightarrow \Gamma \exists \Gamma$ and so on $\Gamma$ as derived notations. The emantics 
is defined in the obvious way. For the deduction system Twe take for example the one of Figure 10.

The basic arithmetical axioms BAA are the universally quantified closures of:

$$
\begin{aligned}
& s(x) \neq 0 \\
& s(x)=s(y) \Rightarrow x=y \\
& x+0=x \\
& x+s(y)=s(x+y) \\
& x * 0=0 \\
& x * s(y)=x * y+y \\
& \neg x<0 \\
& x<s(y) \Rightarrow x<y \vee x=y
\end{aligned}
$$

and the restricted induction axiom $\mathrm{RIA}_{0}$ :

$$
\begin{array}{rl}
\forall_{2} & X \cdot 0 \in X \wedge \\
& \left(\forall_{1} x \cdot x \in X \Rightarrow s(x) \in X\right) \\
\Rightarrow & \forall_{1} x \cdot x \in X
\end{array}
$$

$\mathrm{ACA}_{0}$ is the theory axiomatized by BAAT RIA ${ }_{0} \Gamma$ plus the arithmetical comprehension scheme Texpressing that for every arithmetical formula $F$ (i.e. $\Gamma$ formula without any second-order quantifier $\Gamma$ but possibly with free first-order and set variables) and for every first-order variable $x$ :

$$
\exists_{2} X \cdot \forall_{1} x \cdot(x \in X \Leftrightarrow F)
$$

holds.

We encode this in ramified type theory with operators by letting the operators $\Gamma$ with their signatures $\Gamma$ be:

$$
\begin{array}{ll}
s: \iota \Rightarrow \iota & \neg: o \Rightarrow o \\
+: \iota \times \iota \Rightarrow \iota & \wedge: o \times o \Rightarrow o \\
: \iota \times \iota \Rightarrow \iota & \left.\forall{ }_{1}^{\ell}:(\iota \iota) \circ\right) \Rightarrow o \\
=: \iota \times \iota \Rightarrow o & \\
<: \iota \times \iota \Rightarrow o &
\end{array}
$$

The constants are:

$$
\begin{aligned}
& 0: \iota / 0 \\
& \forall 2:\left((\iota \stackrel{\ell}{\rightarrow} o)^{\ell+1} \rightarrow o\right) \stackrel{\ell+2}{\rightarrow} o / \ell+3
\end{aligned}
$$

where $\ell \geq 1$. The variables are $x_{l}^{0} \Gamma y_{l}^{0} \Gamma z_{l}^{0} \Gamma \ldots$ (all at level 0$) \Gamma \bar{X}_{\substack{0 \\ \rightarrow 0}}^{1} \Gamma \ldots$ (set variables $\Gamma$ all at level 1 ).

The atomic formulas $s \in X$ are encoded as applications $X_{\substack{0 \\ \rightarrow 0}}^{1} s \Gamma$ of type $o / 1$. The arithmetical comprehension axiom is simply encoded as the use of $\lambda$ abstraction. Indeed $\Gamma$ any arithmetical formula $F$ has type $o / 0$ or $o / 1 \Gamma$ hence $\lambda x \cdot F$ has type $\iota \stackrel{0}{\rightarrow} o / 1$. It follows that we can deduce $\exists_{2} X \cdot \forall_{1} x \cdot x \in X \Leftrightarrow F \Gamma$ because $X$ has the right type and level. BAA is encoded in the obvious wayTand RIA $_{0}$ as the following formula $\mathrm{RIA}_{k}$ with $k=0$ :

$$
\begin{aligned}
\forall_{2}^{k} & X^{k+1} \cdot X 0 \wedge \\
& \left.\stackrel{\iota k}{\rightarrow} \vec{\forall}_{1}^{k} x_{\iota}^{k} \cdot X x \Rightarrow X(s(x))\right) \\
\Rightarrow & \forall_{1}^{k} x_{\iota}^{k} \cdot X x
\end{aligned}
$$

By similar arguments $\Gamma$ it follows that $\Pi_{k}^{1}-\mathrm{CA}_{0} \Gamma$ the subsystem of second-order Peano arithmetic where the comprehension axiom is now restricted to $\Pi_{k}^{1}$ formulasTcan be encoded in ramified type theory with operators $\Gamma$ by using $\mathrm{RIA}_{k}$ instead of $\mathrm{RIA}_{0} \cdot \Pi_{1}^{1}-\mathrm{CA}_{0}$ is already stronger than Friedman's system $\mathrm{ATR}_{0}$ [Sim85] Twhich is strong enough to prove the BolzanoWeierstrass theorem and is equivalent in strength to Kruskal's Lemma.

Mechanising proof search in such systems is not the purpose of this paper. We only mention the main difficulties in doing so. The first difficult point is the failure of the subformula property: this is already a problem in the simple theory of types 5 but it already plagues usual formalisations of first-order Peano arithmetic. In fact Tto do any serious mathematics probably involves tackling this difficult problem.

The second difficulty is the fact that $\Gamma$ although $\Pi_{1}^{1}-\mathrm{CA}_{0}$ is strong enough to prove most theorems of everyday mathematics $\Gamma$ it does so in quite contrived ways: we must encode pairs of integers as integers $\Gamma$ sequences of integers as integers $\Gamma$ even inner models of the theory inside the theory. This difficulty is probably only apparent. Nothing prevents us indeed from formalising a richer theory with an explicit pairing operator:

$$
(-,-): \iota \times \iota \Rightarrow \iota
$$

In fact Tmost any inductive datatype that crops up in computer science can be endowed with a theory $\dot{a} l a$ $\mathrm{ACA}_{0}$ or $\Pi_{k}^{1}-\mathrm{CA}_{0}$. For example $\Gamma$ to encode lists of integers $\Gamma$ we add the following operator:

$$
\text { cons }: \iota \times \iota \text { list } \Rightarrow \iota \text { list }
$$

and the constant $n i l: \iota$ list Wwhere $\iota$ list is a new base type. We also add an induction axiom on lists $\Gamma$ restricted in the same way that $\mathrm{RIA}_{k}$ was restricted:

$$
\begin{aligned}
& \forall_{2}^{k} X_{\iota}^{k+1} \operatorname{list}_{\rightarrow \infty}^{k} \cdot X \text { nil^ } \\
& \left(\forall_{1}^{k} x^{k}{ }_{\text {list }}^{\circ}, y_{L}^{k} \cdot X x \Rightarrow X(\operatorname{cons}(y, x))\right) \\
& \Rightarrow \forall_{1}^{k} x_{\text {list }}^{k} \cdot X x
\end{aligned}
$$

The shape of the induction axioms is entirely determined from the definition of the datatype $\Gamma$ here $\iota$ list $::=n i l \mid \operatorname{cons}(\iota, \iota$ list $)$ : see [GLT89]. Moreover $\Gamma$ considering $\iota$ list not as a new base type but as the application of a type operator list to the base type $\iota \Gamma$ thus allowing other list types and a limited form 
of polymorphism $\mathrm{is}$ a benign extension to the type system.

We therefore believe that ramified type theory with operators is a sensible starting point for formalizing powerful enough systems of mathematicsTin an automated deduction perspective.

\section{Conclusion}

We have proposed a formalization of the pure ramified theory of types through a typed $\lambda$-calculus that is simple $\Gamma$ rigorous $\Gamma$ and arguedly in the spirit of usual ramified theories with cumulative levels. We have shown that unification or rather pre-unification $\Gamma$ in such pure theories with integer levels was decidable. However Tthe logical systems for which they can provide foundations are too weak to express any nontrivial logical facts. Extending the frameworks with operators makes the unification problem undecidableI already at order $2 \Gamma$ but the decidability result above should be taken as an indication that ramified type theory with operators is a computationally more sensible basis for automated deduction than simple type theory. It is all the more sensible as most theorems of everyday mathematics can be proved in theories that are formalizable in such ramified systems.

\section{References}

[And86] Peter B. Andrews. An Introduction to Mathematical Logic and Type Theory: To Truth through Proof. Computer Science and Applied Mathematics. Academic Press 1986.

[Bar84] Henk Barendregt. The Lambda Calculus, Its Syntax and Semantics Tvolume 103 of Studies in Logic and the Foundations of Mathematics. North-Holland Publishing CompanyГ Amsterdam $\Gamma 1984$.

[Chu40] Alonzo Church. A formulation of the simple theory of types. Journal of Symbolic Logic 5:56-68Г1940.

[Chu76] Alonzo Church. Comparison of Russell's resolution of the semantical antinomies with that of Tarski. Journal of Symbolic Logic 41(4):747-760ГDecember 1976.

[Cop71] Irving M. Copi. The Theory of Logical Types. Monographs in modern logic. London usw. Routledge and Kegan PaulГ1971.
[Der87] Nachum Dershowitz. Termination of rewriting. Journal of Symbolic Computation $\Gamma$ 3:69-116Г1987.

[Fit90] Melvin C. Fitting. First-Order Logic and Automated Theorem Proving. Springer $\Gamma$ 1990. Second edition 1996.

[Gal91] Jean Gallier. What's so special about Kruskal's Theorem and the ordinal $\Gamma_{0}$. A survey of some results in proof theory. $A n$ nals of Pure and Applied Logic Г53(3):199260 TSeptember 1991.

[GLT89] Jean-Yves GirardT Yves Lafont $\Gamma$ and Paul Taylor. Proofs and Types Tvolume 7 of Cambridge Tracts in Theoretical Computer Science. Cambridge University PressT1989.

[Gol81] Warren D. Goldfarb. The undecidability of the second-order unification problem. Theoretical Computer Science $\mathrm{T} 13: 225-230 \Gamma$ 1981.

[Haz83] Allen Hazen. Predicative logics. In D. Gabbay and F. GuentherTeditorsT Handbook of Philosophical Logic I: Elements of Classical Logic Tchapter I.5Tpages 331-407. D. Reidel Publishing CompanyTDordrecht $\Gamma$ The NetherlandsT 1983. (Synthese library Volume 164).

[Hin69] J. R. Hindley. The principal type scheme of an object in combinatory logic. Transations of the American Mathematical Society $\Gamma 146: 29-60 \Gamma 1969$.

[Hue75] Gérard P. Huet. A unification algorithm for typed $\lambda$-calculus. Theoretical Computer Science $1: 27-57 \Gamma 1975$.

[Koh95] Michael Kohlhase. Higher-order tableaux. In Workshop on Theorem Proving with Analytic Tableaux and Related Methods $\Gamma$ 1995.

[LN95] Twan Laan and Rob Nederpelt. A formalization of the ramified type theory. 26 pages. Available from the author at laan@win .tue.m1 1995.

[McH90] James A. McHugh. Algorithmic Graph Theory. Prentice-Hall InternationalГ1990.

[Mil78] Robin Milner. A theory of type polymorphism in programming. Journal of Computer and System SciencesГ17:348-375Г1978. 
[SG89] W. Snyder and J. Gallier. Higher order unification revisited: Complete sets of tranformations. Journal of Symbolic Computation $\Gamma$ 8(1 \& 2):101-140Г1989. Special issue on unification. Part two.

[Sim85] Stephen G. Simpson. Reverse mathematics. In A. Nerode and R. A. ShoreTeditorsT Recursion Theory $\Gamma$ pages 461-471. American Mathematical SocietyГ1985. Proceedings of Symposia in Pure MathematicsTvol. 42.

[WR27] Alfred North Whitehead and Bertrand Russell. Principia Mathematica. Cambridge University PressГ1910Г1927. 